\title{
Cardioprotective Actions of Relaxin
}

\author{
by \\ Brian Joseph Martin \\ BS, University of Utah, 2014
}

\begin{abstract}
Submitted to the Graduate Faculty of The Swanson School of Engineering in partial fulfillment of the requirements for the degree of Doctor of Philosophy
\end{abstract}

University of Pittsburgh 


\title{
UNIVERSITY OF PITTSBURGH
}

\author{
Swanson School of Engineering
}

This dissertation was presented

by

Brian Joseph Martin

It was defended on

March 13, 2019

and approved by

Guillermo Romero, Ph.D., Associate Professor

Department of Pharmacology and Chemical Biology

Sanjeev Shroff, Ph.D., Professor

Department of Bioengineering

Partha Roy, Ph.D., Associate Professor

Department of Bioengineering

Dissertation Director: Guy Salama, Ph.D., Professor

Department of Medicine 
Copyright $@$ by Brian Martin

2019 


\title{
Cardioprotective Actions of Relaxin
}

\author{
Brian Martin, Ph.D. \\ University of Pittsburgh, 2019
}

Relaxin, a hormone of pregnancy, has shown broad cardioprotective effects including anti-fibrotic (reversed excess TGF $\beta$ signaling), anti-arrhythmic (Nav1.5 and $\mathrm{I}_{\mathrm{Na}}$ upregulation and Cx43 phosphorylation and trafficking to intercalated disks) and anti-inflammatory properties (reduced IL-1 $\beta$ and IL-6). While relaxin's anti-fibrotic effects are thought to occur through the SMAD2/3/TGF $\beta$ pathway, there is a general lack of understanding of relaxin's mode of action to increase sodium current and alter connexin43 localization to suppress arrhythmias. Based on a rat model of aging, we tested the hypothesis that relaxin acts through activation of $\mathrm{Wnt} / \beta$ catenin signaling to mediate its effects on Nav1.5 and Cx43 and to regulate collagen expression. We show for the first time that relaxin activates canonical Wnt signaling (increased nuclear $\beta$ catenin) to increase Nav1.5 in isolated cardiomyocytes. Block of canonical Wnt signaling (via Dikkopf-1) abrogated relaxin's effect on both Nav1.5 in cardiomyocytes and suppression of excess collagen from fibroblasts. Further, we show that relaxin significantly suppressed expression of Dikkopf-1 in isolated cardiomyocytes and whole LV tissue. In addition, show that relaxin suppressed the age-associated genetic upregulation in inflammatory markers in a sexdependent manner. Finally, we tested the hypothesis that relaxin would be an effective therapy in a rat model of pulmonary arterial hypertension and show that it reduced occlusion of small pulmonary arteries, myocardial and lung fibrosis and reversed the cardiac phenotype of ventricular arrhythmia or arrest. These results suggest that relaxin signals through multiple 
pathways to achieve its myriad effects and that relaxin has great potential as a therapy for multiple cardiopulmonary diseases. 
Table of Contents

PREFACE]

1.0 INTRODUCTION]

RELAXIN

1.2 RELXIN AS AN ANTI-FIBROTIC ...................................................................... 4

R.3 RELAXIN AS AN ANTI-ARRHYTHMIQ ......................................................... 10

RELAXIN SIGNALING PATHWAYS

1.5 RELAXIN AND HEART FAILURE]..................................................................... 16

1.6 RELAXINMIMETICS]............................................................................... 18

I.7 INTRODUCTION CONCLUSION

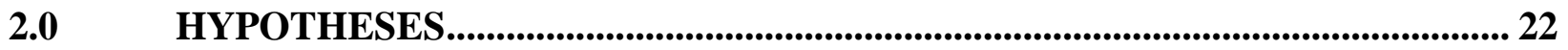

B.0 SPECIFIC AIMS].................................................................................................................. 23

4.0 METHODS

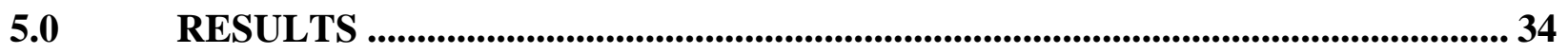

5.1 RELAXIN REVERSES CARDIAC INFLAMMATION].................................. 38

5.2 RELAXIN ACTS THROUGH WNT SIGNALING]......................................... 55

5.3 RELAXIN AS A THERAPY FOR PAH]............................................................... 71

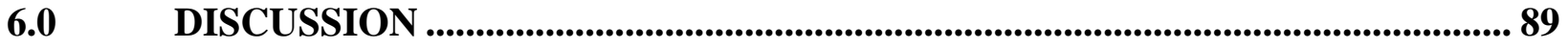


7.0 FUTURE DIRECTIONS AND CONCLUSIONS

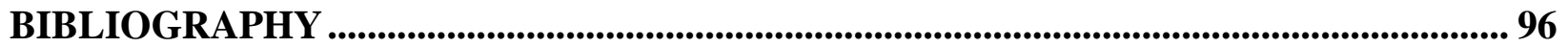




\section{List of Tables}

Table 1. Mechanisms of Collagen Inhibition by Relaxin .............................9

Table 2. RNA-Sequencing Analysis of Cardiovascular Disease Related genes in Aging, 40

Table 3. Upstream Analysis of RNA-sequencing Gene Expression Data................41

Table 4. Chemokine and Chemokine Receptor Transcript Expression. ...................42

Table 5. Common Genes Altered by Relaxin: Female, $. . \ldots \ldots \ldots \ldots \ldots \ldots \ldots \ldots \ldots \ldots \ldots . . .46$ 


\section{List of Figures}

Figure 1. Relaxin Signaling Pathways in Fibroblasts...$\ldots \ldots \ldots \ldots \ldots \ldots \ldots \ldots \ldots \ldots \ldots$

Figure 2. Protein Expression of ANP in Female and Male Hearts.....................41

Figure 3. Volcano Plots of Differently Expressed Gens in Aging and Relaxin...........45

Figure 4. Pathway Analysis: Female...$\ldots \ldots \ldots \ldots \ldots \ldots \ldots \ldots \ldots \ldots \ldots \ldots \ldots \ldots \ldots \ldots \ldots \ldots$

Figure 5. Cellular Function Prediction from Differentially Expressed Female Genes....47

Figure 6. Complement Pathway Activity with Aging and Relaxin: Female..............49

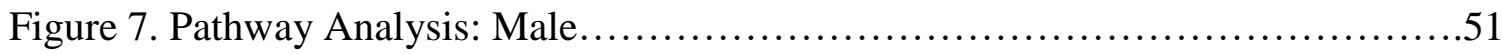

Figure 8. Effect of Aging and Relaxin on Macrophage Accumulation in the Heart.......52

Figure 9. Relaxin Effects on Conduction Velocity and Collagen Expression in Aged

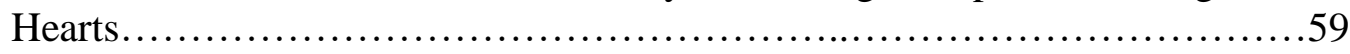

Figure 10. Relaxin Effects on Intercalated Disk Proteins in Left

Ventricle..................................................60

Figure 11. Relaxin Effects on Intercalated Disk Proteins in Atria. .....................61

Figure 12. Relaxin Effects on Dikkopf-1 Expression: Whole Left Ventricle.............62

Figure 13. Relaxin Effects on Nav1.5 in Left Ventricular Cardiomyocytes...............63

Figure 14. Relaxin Signals Through Wnt Signaling to Increase Nav1.5. ................66

Figure 15. Relaxin Effects on Dikkopf-1 Expression: Isolated Cardiomyocytes..........67

Figure 16. Relaxin Effects on Collagen Expression from Fibroblasts..................67

Figure 17. Relaxin-Wnt Interplay Modulates Collagen Expression from Fibroblasts.....68

Figure 18. Relaxin Effects on Wnt1 Expression from Fibroblasts.....................68

Figure 19. Experimental Protocol of Pulmonary Arterial Hypertension Study.............74 
Figure 20. Relaxin/Sugen Effects on Doppler Flow Patterns...$\ldots \ldots \ldots \ldots \ldots \ldots \ldots \ldots \ldots \ldots .77$

Figure 21. Arrhythmia Phenotypes in Pulmonary Arterial Hypertension Hearts...........78

Figure 22. Dithiothreitol Rescues Asystolic Pulmonary Arterial Hypertension Hearts....79

Figure 23. Relaxin Effects on Conduction Velocity in Pulmonary Arterial|

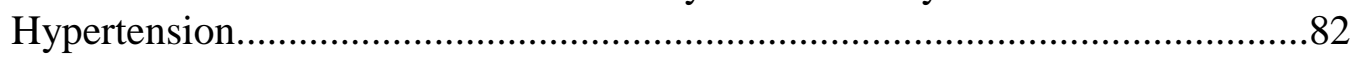

Figure 24. Relaxin Effects on Ventricular and Pulmonary Fibrosis $. . \ldots \ldots \ldots \ldots \ldots \ldots \ldots . . . . . .83$

Figure 25. Histology of Diseased and Relaxin Treated Lungs. .......................85

Figure 26. Occlusion and Plexiform Lesion Quantification of Diseased and Relaxin

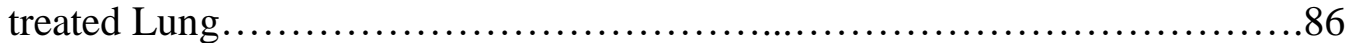

Figure 27. Relaxin Concentration in Maternal and Fetal Plasma ........................90

Figure 28. Relaxin Concentration Correlation Measurements in Maternal and Fetal

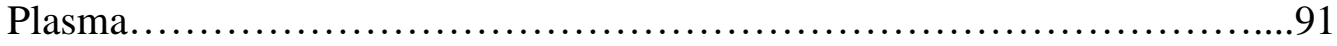




\section{Preface}

This dissertation on the cardioprotective actions and signaling mechanisms of the hormone relaxin, has been five years in the making. While there were high's and low's, positive and negative experiments and sleepless nights, I feel the work contained herein should significantly advance the understanding of the beneficial effects relaxin can have in cardiovascular therapies as well relaxin's underlying mechanism of action in modulation of ion channel expression and reduced fibrosis.

I would like to thank my mentor, Dr. Guy Salama, for his invaluable guidance, direction, knowledge and friendship. He has taught me much and allowed me to develop into the sort of scientist I want to be. Also, I would like to thank Dr. Guillermo Romero for his investment in my development, and Dr. Sanjeev Shroff for his tutelage and support. Collectively, I would like to thank my Ph.D. committee for their valuable insights into my projects, and their willingness to tutor and teach me and to support my work. Further, I want to thank all those with whom I have interacted and who have aided in my progression as a graduate student and scientist.

Most importantly, I would like to thank my wife, Erica, for her constant support, advice, steady guidance and strong belief in me through my time here at the University of Pittsburgh. 


\subsection{Introduction}

\subsection{Relaxin}

Relaxin (RLX) was first discovered by Frederick Hisaw in 1926, when he showed that injection of serum from pregnant guinea pigs into virgin guinea pigs resulted in relaxation of the pubic ligament.[1] The hormone was extracted from the corpus luteum of the pig in 1930 and was officially named RLX.[1] In subsequent years, RLX was shown to be structurally like insulin (53 a.a.), containing conserved cysteine residues, an A and a B chain and two inter- and one intra-chain disulfide bond.[1, 2, 3] Humans (and other primates) have three RLX genes (RLN1-3) encoding for RLX 1-3, though lower mammals (e.g., mice) have only RLN1 and RLN3. RLN1 in lower mammals is equivalent to RLN2 in humans and other primates and encode for the most prominent circulating RLX in the body (hence, RLX2 in humans and RLX1 in rodents will be referred to herein as RLX). RLN3 encodes for RLX-3 which is primarily expressed in the brain of mammals.[1] Cloning techniques and database searches led to the discovery of several insulin-like peptides (INSL) 3-6, of which INSL-3 is expressed highly in Leydig cells and probably plays a role in testis descent in males and fertility in females, much less is known about INSL 4-6.[1]

The primary RLX receptors are classified as the relaxin family peptide receptors (RXFP) 1-4 and are G-protein coupled receptors (GPCR). RXFP1, the primary receptor for RLX, was 
deorphanized in 2002 through recognition that RLX increased cAMP signaling, a mechanism prominently linked to GPCRs.[1, 34 ] In addition to a seven-transmembrane domain, a prominent feature of GPCRs, RXFP1 contains an extracellular leucine rich repeat domain connected to the transmembrane domain by a cysteine-rich hinge [2], and also contains a low-density lipoprotein receptor type A (LDLa) module connected to the LRR through a linker.[1]

Activation of RXFP1 by RLX is thought to involve binding of the RLX B-chain to the LRR and interaction of the LDLa-LRR linker with the RLX A chain $[1,5]$ and exoloops of the transmembrane domains. In sum, the binding of RLX to RXFP1 involves complex interactions which makes it challenging to develop RLX mimetics RLX was extensively studied as a hormone of pregnancy and was found to play a critical role in several aspects of the reproductive process; to name a few: a) softening of the birth canal, probably due to its regulation of the extracellular matrix components, b) decidualization, c) implantation, d) suppression of uterine contractions e) regulation of nipple development, increased maternal renal plasma flow, glomerular filtration, and cardiac output.[6, 7] Finally, it should be noted that RLX and RXFP1 expression and localization vary as a function of species and sex [1], and may have gender specific roles during inflammation.[8]

There is a growing body of evidence that indicates a still broader range of actions by RLX beyond those of reproduction. For instance, its receptor, RXFP1 has been found to be expressed in a myriad of tissues and organs including ovaries, mammary glands, testis, prostate, brain, liver, kidney, lung, vasculature and heart.[1, 9, 10] Moreover, exogenously added RLX appears to exert positive/beneficial genomic effects in many of these organs. $[8,11,12,14,15]$ In clinical trials, RLX was well tolerated with no adverse effects in heart failure patients.[16] Interestingly, serum RLX levels are increased in patients (males and females) with particular 
diseases: prostate cancer [17], pulmonary arterial hypertension [18], atrial fibrillation and heart failure. Many of these pathologies are associated with a common set of risk factors (e.g. age, hypertension, myocardial ischemia, fibrosis and inflammation).[19, 20, 21] It is important to note that there is a significant controversy regarding he accuracy and validity of commercially available RLX assay kits which casts doubt on the interpretation of the data and some of these studies will have to be revisited with new assays that detect RLX concentrations more reliably.[22]

As shown by Debrah et al, [23, 24, 25] RLX treatment resulted in marked cardiovascular effects: increased cardiac output and arterial compliance, decreased arterial load, reduced collagen expression and beneficial remodeling of the vascular wall.

The primary goal of this introduction is to give an overview of RLX-mediated cardioprotective actions and will only touch briefly on RLX effects in other organs. However, a discussion of RLX's signaling mechanisms will be centered on findings in neonatal and noncardiac cells, since little work has been done in adult primary cardiac cells, a deficit that our lab is trying to remedy. The review will include a discussion of RLX's signaling mechanisms in fibroblasts and myocytes, its anti-arrhythmic properties, and anti-inflammatory properties. The review will also present a brief history of RLX's use in clinical trials for patients with acute decompensated heart failure and recent attempts at developing RLX mimetics. 


\subsection{Relaxin As an Anti-fibrotic}

In response to injury or disease, inflammatory cells are recruited to the site of injury for tissue repair, wound closure or pathogen removal. Pro-fibrotic and inflammatory cytokine levels (e.g. TGF $\beta 1$, Angiotensin II) are increased and activate fibroblasts to their collagen secreting phenotype, the myofibroblast.[26] The inability of the body to resolve the injury or remove the source of infection can cause prolonged activation of fibroblasts resulting in pathological collagen accumulation and myocardial stiffness, impaired systolic and diastolic function and increased arrhythmogenicity. Atrial and ventricular fibrosis may increase the arrhythmia risk due to a direct increase in extracellular resistance and an increase in the tortuosity of the extracellular matrix.

Based on mathematical simulations the effects of fibrosis on conduction velocity are not significant if based on changes in extracellular resistance or extracellular tortuosity.[27] A more significantly effect comes about from the trans-differentiation of fibroblasts to myofibroblasts as the latter express connexin 43 which may form gap-junctions between myofibroblasts and myocytes. Such heterotypic junctions would tend to depolarize the resting potential of myocytes, slow-down the action potential upstroke and conduction velocity of the action potential, resulting in reentrant electrical circuits. Such heterotypic cell-cell coupling could be demonstrated in cocultures of fibroblasts and myocytes [28] but direct evidence of their occurrence in an infarct was lacking. A more recent study demonstrated that in mice with a cryoablated infarct, myocytes formed heterotypic junctions with cells in the myocardial scar.[29] While fibrosis promotes arrhythmia, [30] an important question is what promotes pathological fibrosis. Several factors have been associated with increases in fibrosis: increased cardiac afterload, cell death or cell stretching can lead to release of pro-fibrotic cytokines from cardiomyocytes and fibroblasts, 
resulting in fibroblast activation and pathological collagen deposition.[31] In addition, fibrosis can also be idiopathic with no known instigating trigger.[32]

In addition to the pro-fibrotic cytokines TGF $\beta$ and Ang II, Wnt signaling has emerged as a player in fibroblast activation and fibrosis. In the absence of canonical Wnt ligands, cytosolic $\beta$-catenin is phosphorylated and marked for ubiquitination by the destruction complex comprising GSK3 $\beta$, Adenomatosis Polyposis Coli, casein kinase 1 and Axin. During canonical Wnt signaling, Wnt ligands bind to frizzled receptors and co-receptors, lipoprotein receptorrelated protein (LRP) 5/6, which leads to disassembly of the destruction complex, and translocation of $\beta$-catenin into the nucleus, where it acts as a co-transcription factor with TCF and LEF.[33] Canonical Wnt signaling has multiple native inhibitors, the Dickkopf (DKK) 1-4 proteins, the secreted frizzled related proteins (sFRP) 1-5 and Wnt inhibitor factor (WIF)-1.[34] Studies have shown that canonical Wnt signaling in fibroblasts results in increased collagen expression in human and mouse dermal fibroblasts through increased Wnt1 and decreased DKK1 expression.[32] Similarly, He et al [35] showed that addition of sFRP2 resulted in reduced collagen production by cardiac fibroblasts in 6-week old Sprague Dawley rats, while Kobayashi et al [36] report that sFRP2 results in increased collagen and that sFRP2 null mice show less fibrotic scar after MI. These conflicting sFRP2 results can partially be reconciled in that sFRP2 appears to exhibit a biphasic response and that at low concentrations, as used in the Kobayashi report, sFRP2 is pro-fibrotic and at higher doses, such as used in the He study, sFRP appears to be anti-fibrotic. Further, Blyszczuk et al [37] showed in a model of experimental autoimmune myocarditis, that inflammatory CD133+ cells convert to myofibroblasts in the presence of TGF $\beta$, and that this effect is dependent on active Wnt signaling. They showed that inhibition of Wnt signaling via high dose of sFRP2 blocks activation of fibroblasts, though they do not show 
results with low dose sFRP2. In sum, Wnt signaling appears to be intricately involved in fibroblast activation, collagen secretion and pathological fibrosis, though further work exploring dose dependence and time-course treatments should be performed to better understand the interplay between TGF $\beta$ and Wnt signaling.

Fibrosis is a common component of many cardiovascular diseases including hypertension [11, 14], myocardial infarction [38], cardiac arrhythmogenesis [13, 14] and normal, 'healthy' aging.[8, 39] More recently, RLX has emerged as a potent antifibrotic in cardiac tissue and isolated fibroblasts, as numerous studies showed that RLX reverses cardiac fibrosis in hypertensive, arrhythmogenic and aged rats $[11,13,14,40]$, as well as diabetic cardiomyopathy [41] and myocardial infarction.[38]. In agreement with these findings, RLX-/- mice develop multi-organ fibrosis at an early age compared to RLX+/+ mice, although cardiac fibrosis is sexdependent, being pronounced in RLX-/- male but not female mice.[42]

As mentioned, fibroblast activation is characterized by increased stress fiber formation and elevated $\alpha$-smooth muscle actin and collagen expression, a process shown to be inhibited by RLX.[43] Pioneering studies by Unemori and Amento in dermal [44] and lung fibroblasts showed that RLX (10 and $100 \mathrm{ng} / \mathrm{mL}$ ) significantly increased the expression of pro-collagenase and decreased expression of the procollagenase inhibitor, the tissue inhibitor of metalloproteinases (TIMP), and the expression of collagen mRNA and protein. Further work by Palejwala et al $[45,46]$, using human lower segment uterine fibroblasts from full-term women confirmed the effects of RLX (1 and $10 \mathrm{ng} / \mathrm{mL}$ ) on pro-collagenase expression and an increase in MMP-2 and MMP-3, while downregulating TIMP-1. Interestingly, they showed that RLX significantly increased protein tyrosine phosphorylation and that RLX mediated effects on MMP-1 and MMP-3 were c-Raf (serine/threonine kinase) dependent. 
Studies in recent years have led to partial elucidation of the mechanism by which RLX blocks fibroblast activation and collagen secretion. Samuel et al [43] showed that cardiac fibroblasts from 1-day old Sprague Dawley (SD) rats treated with TGFß1 (1-2 ng/mL) or Angiotensin II (Ang II, 1-5X10-7 M) for 72-hours resulted in a significant increase in collagen expression in atrial and ventricular fibroblasts that was inhibited by RLX (100 ng/mL). In addition, they showed that Ang II stimulated fibroblast proliferation was significantly blocked by RLX treatment.

RLX did not significantly alter baseline levels of collagen yet suppressed pathological collagen, suggesting that RLX blocks the action of certain cytokines that activate fibroblasts rather than altering collagen expression directly. Nevertheless, male 12-month-old RLX-/- mice exhibit significantly higher levels of ventricular fibrosis compared to littermate controls, and RLX treatment (500 $\mu \mathrm{g} / \mathrm{kg} / \mathrm{day}$ for 2-weeks) significantly reduced collagen accumulation (Samuel et al., 2004). Based on a cAMP bioassay, there was no correlation between RLXdependent suppression of fibrosis and cAMP levels in fibroblasts suggesting that RLX regulation of fibrosis was not mediated by an increase in cAMP.[43] RLX's anti-fibrotic mechanism was elucidated further in a study by Heeg et al [47] using two renal fibroblast cell lines. They showed that RLXs anti-fibrotic properties are mediated through an inhibition of TGF $\beta$, and an inhibition of SMAD2 phosphorylation and nuclear translocation. Mookerjee et al [48] confirmed the effects seen by Heeg et al and showed that inhibition of nitric oxide (NO) signaling, primarily nNOS and cGMP, significantly abrogated RLX's anti-fibrotic effects. Sassoli et al [49] expanded these studies by showing that in NIH/3T3 fibroblasts, and in fibroblasts isolated from 1-day old CD1 albino mice, TGF $\beta$ reduced the expression of the Notch-1 receptor and its ligand, Jagged-1, and both effects were reversed by RLX. RLX significantly decreased SMAD3 phosphorylation and 
inhibition of Notch signaling increased pSMAD3 and abrogated RLXs effect. Similar findings were observed in human umbilical vascular endothelial cells where inhibition of Notch-signaling significantly blocked the effects of RLX on cell migration and endothelial-mesenchymal transition (EndoMT).[50]

Besides fibrosis, activation of fibroblasts can result in the expression of ionic channels and currents.[51] The electrical properties of activated fibroblast may have significant consequences on cardiac arrhythmias because these fibroblasts may couple electrically with neighboring cardiomyocytes via functional gap junctions [52, 53], depolarize these cardiomyocytes and alter their electrical activity.[55] A study by Li et al [56] showed that small percentages of human cardiac fibroblasts exhibit $\mathrm{K}+\mathrm{Cl}$ - and $\mathrm{Na}+$ currents, but do not mention if expression of ionic currents correlated with normal fibroblasts or with myofibroblasts. Chatelier et al [57] and Koivumaki et al [58], suggest that $\mathrm{Na}+$ channel proteins and $\mathrm{Na}+$ currents are measurable in myofibroblasts but not in fibroblasts. Squecco et al [51] showed that compared to control, TGF $\beta$ treated fibroblasts had significantly lower (more negative) resting membrane potentials and increased delayed rectifier $\mathrm{K}+$ current $\left(\mathrm{I}_{\mathrm{KDR}}\right)$. In addition, TGF $\beta$ increased the $\mathrm{Ca} 2+$ dependent $\mathrm{K}+$ current (IBK), the inward rectifying $\mathrm{K}+$ current $\left(\mathrm{I}_{\mathrm{Kir}}\right)$ and the slow component of the delayed rectifier $\mathrm{K}+$ current $\left(\mathrm{I}_{\mathrm{Ks}}\right)$ compared to untreated controls. RLX significantly blocked TGF $\beta$-induced increases in each of these currents, suggesting that RLXs anti-fibrotic effects may involve inhibition of fibroblast activation by blocking ion channel expression and current. Further work suggests that RLX blocks TGFß-mediated STAT3 phosphorylation and autophagy [59], and may signal through $\mathrm{PKC} \delta$ to mediate its effects in diabetic cardiomyopathy.[60] The inhibitory mechanisms of RLX on fibroblast activation are summarized in Table 1 and Figure 1. 
Table 1: Mechanisms of Collagen Inhibition by Relaxin.

\begin{tabular}{|c|c|c|c|}
\hline Fibroblast Source & RLX Anti-Fibrotic Effect & RLX Mechanism in Fibroblast & References \\
\hline $\begin{array}{c}\text { Dermal Fibroblasts (human) } \\
\text { Lung Fibroblast (adult } \\
\text { human) }\end{array}$ & $\begin{array}{c}\downarrow \text { TGF } \beta \text { mediated collagen and } \\
\text { fibronectin expression } \\
\downarrow \text { TIMP } \\
\uparrow \text { MMP-1 }\end{array}$ & N/A & $\begin{array}{l}\text { Unemori and Amento. 1990; } \\
\text { Unemori, et al, } 1996\end{array}$ \\
\hline $\begin{array}{l}\text { Lower Uterine Segment } \\
\text { Fibroblasts (full-term human) }\end{array}$ & $\begin{array}{c}\downarrow \text { TIMP } \\
\uparrow \mathrm{MMP}-1, \text { MMP-2, MMP-3 }\end{array}$ & $\begin{array}{c}\text { Tyrosine Phosphorylation } \\
\text { c-Raf dependent } \\
\text { PKC and cAMP independent }\end{array}$ & Palejwala et al. 2001 \\
\hline $\begin{array}{l}\text { Cardiac Fibroblasts (1-day } \\
\text { old, SD Rat) }\end{array}$ & $\begin{array}{c}\downarrow \mathrm{TGF} \beta / \text { Ang II mediated collagen } \\
\text { secretion } \\
\downarrow \text { Autophagy }\end{array}$ & $\begin{array}{c}\text { No change in cAMP } \\
\downarrow \text { STAT3 phosphorylation and } \\
\text { autophagy } \\
\uparrow \mathrm{PKC} \delta \text { expression }\end{array}$ & $\begin{array}{l}\text { Samuel et al. 2004; Yuan et } \\
\text { al. 2017; Wang et al. } 2017\end{array}$ \\
\hline $\begin{array}{l}\text { TK-173, TK188 Renal } \\
\text { Fibroblast Cell Lines }\end{array}$ & $\begin{array}{c}\downarrow \text { TGF } \beta \text {-mediated fibroblast } \\
\text { differentiation }(\downarrow \text { QSMA) } \\
\downarrow \text { Collagen expression } \\
\uparrow \text { MMP- } 2 / 9\end{array}$ & $\begin{array}{c}\uparrow \text { Tyrosine phosphorylation } \\
\text { TGF } \beta \text { receptor binding in unaffected } \\
\downarrow \text { SMAD2 phosphorylation } \\
\downarrow \text { SMAD2 nuclear translocation } \\
\text { No effect on SMAD3/7 translocation } \\
\text { MAP kinases (JNK1/2, ERK and } \\
\text { p38) phosphorylation unaffected }\end{array}$ & Heeg et al. 2005 \\
\hline $\begin{array}{l}\text { Primary Renal Cortical } \\
\text { Fibroblasts (3-days post } \\
\text { unilateral ureteric } \\
\text { obstruction) }\end{array}$ & $\begin{array}{l}\downarrow \text { fibroblast differentiation } \\
\qquad(\downarrow \alpha \text { SMA })\end{array}$ & $\begin{array}{c}\text { RXFP1 necessary for anti-fibrotic } \\
\text { effect } \\
\downarrow \text { SMAD2 phosphorylation } \\
\uparrow \text { ERK1/2 phosphorylation } \\
\text { RLX signals through nNOS-NO- } \\
\text { cGMP } \\
\downarrow \text { SMAD2 phosphorylation blocked } \\
\text { by inhibition of nNOS or cGMP. }\end{array}$ & Mookerjee et al. 2009 \\
\hline $\begin{array}{l}\text { NIH/3T3 and CD1 albino } \\
\text { mouse (1-day old) }\end{array}$ & $\begin{array}{c}\downarrow \text { Stress fiber formation } \\
\downarrow \text { Fibroblast differentiation } \\
(\downarrow \alpha \text { SMA }) \\
\downarrow \text { TGF } \beta \text { mediated collagen } \\
\text { secretion } \\
\uparrow \text { MMP- } 2 / 9 \\
\downarrow \text { TIMP2 } \\
\end{array}$ & $\begin{array}{c}\text { Notch signaling negatively regulates } \\
\text { TGF } \beta / \text { SMAD3 } \\
\downarrow \text { Notch Signaling by TGF } \beta \\
\uparrow \text { Notch signaling by RLX } \\
\downarrow \mathrm{K}^{+} \text {currents by RLX }\end{array}$ & $\begin{array}{c}\text { Sassoli et al. 2013; Squecco et } \\
\text { al. } 2015\end{array}$ \\
\hline
\end{tabular}


Note that the studies outlined above were performed with non-cardiac fibroblasts, or neonate cardiac fibroblasts, and none studied RLX's effect on adult cardiac fibroblasts. It is therefore unclear that the results seen in neonates or immortalized cell lines will translate to adult cardiac cells, and further work should be performed in this area.

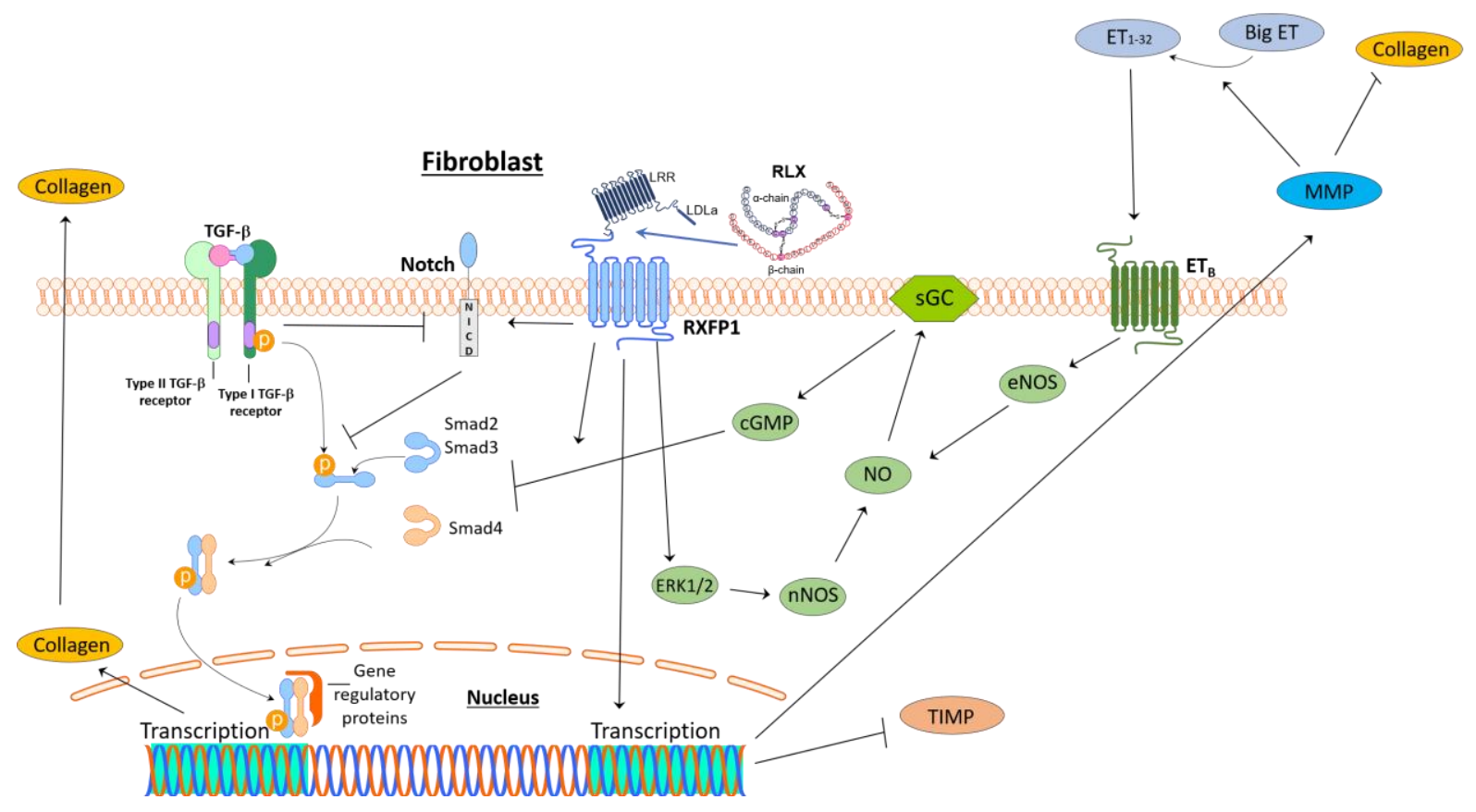

Figure 1: Relaxin Signaling Pathways in Fibroblasts. Collagen suppressing actions of Relaxin in fibroblasts. Relaxin inhibits TGF $\beta$ induced collagen overexpression through inhibition of SMAD2 phosphorylation and activation of the Notch signaling pathway. Further, relaxin has been shown to increase matrix metalloproteinase (MMP) expression to cleave big Endothelin (ET) to ET1-32, leading to increased nitric oxide (NO) signaling and suppressing collagen expression. Inhibition of NO signaling has been shown to significantly impede relaxin's collagen suppressive effects. RLX: relaxin. RXFP1: relaxin family peptide receptor 1. LRR: leucine rich repeat. LDLa: low density lipoprotein A module. NICD: activated intracellular Notch-1; TIMP. Tissue inhibitor of metalloproteinase.

\subsection{Relaxin As an Anti-Arrhythmic}

Atrial fibrillation (AF) is the most common cardiac arrhythmia, increases with age and is characterized by uncoordinated contractions of the atria, resulting in blood clot formation and 
stroke[13, 14, 62]During normal sinus rhythm, the pacemaker action potential is initiated in the sinoatrial (SA) node, propagates across the right and left atria, is delayed at the atrioventricular node, and propagates through the specialized conduction system then throughout the right and left ventricles. In each cell, the action potential controls the myocyte contraction and except for SA nodal cells, normal, healthy myocytes do not fire action potentials spontaneously.[62] Supraventricular arrhythmias, like atrial fibrillation, impede ventricular contractions and ejection fraction slightly but result in a high-risk for stroke, and if untreated increase the vulnerability to heart failure. Electrical (e.g. ion channel expression) and structural (e.g. extracellular matrix) remodeling in aging, $\mathrm{AF}$ and $\mathrm{HF}$ alter the cardiac substrate and promote spontaneous APs that are out-of-phase with the intrinsic beat controlled by the SA node. Extra or premature APs can propagate as they encounter excitable tissue created by anatomical (MI) and/or dynamic blocks due to gradients of repolarization. These conditions lead to triggered activity and reentrant circuits that sustain fibrillation. Several factors can initiate arrhythmias [63] including spontaneous $\mathrm{Ca} 2+$ release from the sarcoplasmic reticulum resulting in delayed after depolarizations, $[64,65]$ through hyper-phosphorylation of ryanodine receptors by protein kinase A (PKA) [66] or Ca/calmodulin-dependent kinase II [67, 68], AP prolongation via increased $\mathrm{Ca} 2+$ influx and decreased $\mathrm{K}+$ currents $[69,70]$, gap junction remodeling and reduced sodium current $\left(\mathrm{I}_{\mathrm{Na}}\right)$ causing reduced AP conduction velocity $(\mathrm{CV})[63,71,72]$, and cardiac fibrosis resulting in AP conduction block.[62]

The first studies looking at RLXs effects on cardiac electrophysiology came from observations of RLXs chronotropic and ionotropic effects.[73] Han et al [73] used isolated cells from rabbit sinoatrial pacemaker cells to show that RLX $(80 \mathrm{nmol} / \mathrm{L})$ caused a significant increase in $\mathrm{I}_{\mathrm{Ca}, \mathrm{L}}$ in a PKA dependent manner. 
In two studies of rat atrial cell electrophysiology, Piedras-Renteria et al $[74,75]$ showed that RLX (100 ng/mL) acutely inhibited the transient outward potassium current ( $\left.\mathrm{I}_{\mathrm{TO}}\right)$ in a PKA dependent manner causing a prolongation of AP duration (APD) and stronger contractions presumably due to an increase of $\mathrm{Ca} 2+$ influx due to the prolonged APD.

Spontaneously hypertensive rats (SHR) are known to have a high susceptibility to AF than normotensive controls; Parikh et al [14] showed that RLX (500 $\mu \mathrm{g} / \mathrm{kg} /$ day) dramatically reduced AF susceptibility in SHR hearts. They demonstrated that the interplay of several mechanisms mediated by RLX contributed to AF suppression, including: decreases in fibrosis and cellular hypertrophy and increased conduction velocity (CV) through an increase in voltagegated $\mathrm{Na}+$ current, INa and Connexin 43 phosphorylation. Henry et al [13] extended these findings to aged rat hearts (F-344 Brown/Norway rats). In isolated cardiac myocytes derived from human induced pluripotent stem cells (hiPS-CMs), Parikh et al showed that RLX increased INa after 24 hours, and Henry et al obtained a similar result in adult rat atrial myocytes under voltage-clamp. The higher $\mathrm{I}_{\mathrm{Na}}$ was associated with an increase in SCN5A (gene coding for Nav1.5) mRNA and Nav1.5, the primary subunit of the voltage-gated sodium channel protein, suggesting that RLX acted via a genomic mechanism rather than a post-translational modification of activation/inactivation kinetics of the $\mathrm{Na}+$ channel. In aged rats, this hypothesis was strengthened by RNA-sequencing data [8] showing reduced SCN5A in aging and increased SCN5A expression by RLX in male and female LV [8] (GSE106377). Many of these findings have been confirmed in two studies in a murine myocardial infarction (MI) model [76, 77] showing a significant reduction in AF by RLX $(75 \mu \mathrm{g} / \mathrm{kg} /$ day $)$ after MI compared to control mice, significantly reduced fibrosis in the RLX group and reduced inflammatory markers In a rat model of MI, Wang et al [78] showed that RLX (500 $\mu \mathrm{g} / \mathrm{kg} /$ day $)$ significantly blunted the 
reduction of $\mathrm{Cx} 43$ in the infarcted myocardium and suppressed ventricular tachyarrhythmia. Studies by Bani et al [79] and Nistri et al [80] showed in a rat and swine model of ischemiareperfusion (I/R) injury, respectively, that RLX (200 ng/ml [79] and 2 or $5 \mu \mathrm{g} / \mathrm{kg} /$ body weight [80]) significantly blocked cardiac mast cell activation, histamine release, neutrophil accumulation and reduced oxidative damage. They hypothesize these effects may have contributed to the anti-arrhythmic properties of RLX noted in these I/R models.

While we have shown that RLX significantly increased Nav1.5 expression, $\mathrm{I}_{\mathrm{Na}}$, and Cx43 phosphorylation, the mechanisms underlying these effects remain unknown. A body of recent work suggests that Wnt signaling may play a pivotal role in ion channel and gap junction protein expression and ionic current regulation. Using NRVM cultured with Wnt1 or Wnt4, Ai et al [81] showed that Wnt1 significantly increased Cx43 protein and mRNA and $\beta$-catenin protein, while Wnt4 increased only $\mathrm{Cx} 43$ protein expression, having no effect on $\mathrm{Cx} 43 \mathrm{mRNA}$ or $\beta$-catenin protein.

Work from Liang et al [82] showed that NRVM stimulated with Wnt3a or the Wnt signaling activator, CHIR-99021 (GSK3 $\beta$ inhibitor), resulted in a significant decrease in SCN5a mRNA expression (gene encoding Nav1.5), Nav1.5 protein and $\mathrm{I}_{\mathrm{Na}}$. Interestingly, Kir2.1 mRNA, protein and current were not affected by Wnt3a, nor were currents through L-type Ca2+ channels, or their associated mRNA or protein. The effect of Wnt signaling on Nav1.5 and INa is not certain; however, Asimaki et al [83] showed that activation of canonical Wnt signaling (inhibition of GSK3 $\beta$ with SB216763) prevented reduction of INa and IK1, and normalized expression and localization of the intercalated disk (ID) proteins plakoglobin and Cx43, in zebrafish and NRVM models of arrhythmogenic cardiomyopathy (ACM). These latter findings parallel the phenotype of ACM patients [83]. Murine models of ACM at age 3-weeks and 6- 
months, treated with SB216763 showed marked improvement in fibrosis, inflammation and apoptosis, as well as protection from ventricular ectopy and increased levels of plakoglobin and Cx43 at IDs compared to non-treated ACM mice.[84]

Ongoing work in our lab suggests that a close interplay between RLX and Wnt/ $\beta$ catenin signaling accounts for the higher expression of Nav1.5 and Cx43 trafficking to the ID in adult (9months) and aged (24-months) rat hearts.

\subsection{Relaxin Signaling Pathways}

GPCR-cAMP: Early studies proposed that the RLX receptor could be a tyrosine kinase receptor, due to its effects on protein tyrosine phosphorylation and cAMP accumulation.[46, 85, 86] Hsu et al [3] first reported the leucine rich GPCRs LGR7 (RXFP1) and LGR8 (RXFP2) as the primary RLX receptors. Using 293T cells, they showed that RLX caused this rise in cAMP only when the cells were transfected with LGR7 or LGR8, and further showed that these receptors were present in brain, uterus, ovaries, prostate and the heart, among other organs. Early studies by Nguyen et al $[87,88]$ showed that RLX increased cAMP accumulation in a biphasic manner; the early increase in cAMP (1-2 minutes) is probably due to Gas activation, and a later (10-20 minutes) rise in cAMP was due to phosphoinositide 3-kinase (PI3K) actions on PKC $\zeta$. Halls et al expanded this model to show that the G- $\beta \gamma$ subunit was involved in the delayed activation of PI3K-PKC activity in RXFP1 expressing cells, but not RXFP2 cells.[89] It has been proposed that the RLX-cAMP signaling pathway differs at low RLX concentrations (attomolar vs. nanomolar) normally found in the blood, and involves a signalosome including AKAP97, PKA, PDE4D3 and $\beta$-arrestin-2.[90] Moreover, RLX signaling is cell dependent; for example, 
cAMP does not appear to be involved in RLX mediated fibroblast signaling compared to other cell types studied (e.g. HUVSMC, HEK-293T).[89, 91] Further different cell types exhibit different cAMP responses to RLX and these responses as a function of RLX-dose can be bellshaped, or sigmoidal shaped responses.[91] A further complexity of RLX signaling is that RLX signaling differs in various cell types because it is dependent on the various combinations of different $\mathrm{G}$ proteins in a particular cell.[92, 93] In sum, RLX signaling through GPCRs is highly complex, dependent on cell type and organ specific.

Nitric Oxide and Inflammation: Studies have shown that RLX increases coronary blood flow, is potent vasodilator, and that the nitric oxide (NO)-guanylyl cyclase-cGMP pathway is necessary for various actions of RLX.[94, 95]. Inhibition of NO signaling in a guinea pig model of ischemia-reperfusion and rat and guinea pig mast cells, blocked RLXs beneficial effects on coronary flow, NO production, histamine release, cell damage due to oxidative stress and cardiac contractions.[95, 96]. In bovine aortic smooth muscle cells, RLX was shown to increase inducible nitric oxide synthase (iNOS), cGMP and reduce intracellular calcium concentration.[97]. In addition, inhibition of NF-kB inhibited RLXs effect on NOS expression.[98] RLX blunted the adhesive nature of neutrophils to rat coronary endothelial cells through an NO-dependent mechanism by reducing the adhesion molecules P-selectin and VCAM-1, an effect blocked by NO inhibition [99] and RLX blocked the activation and migration of human neutrophils, and reduced superoxide anion expression in an NO dependent manner.[100]. RLX reduced ET-1 expression, increased the ETB receptor, and cleaved big ET to ET1-32 via a MMP-2 and MMP-9 dependent mechanism, which can activate NO signaling.[101] In addition, RLX increased the ETB receptor in a cell specific manner (e.g. endothelial and epithelial cells, but not smooth muscle cells) [102], though these findings of RLX on ETB 
expression have been challenged.[103] NO has also been implicated in RLX-mediated inhibition of collagen secretion [48] through reductions in TGF $\beta$ signaling and inhibition of fibroblast to myofibroblast transition and increase MMP activity through and nNOS-NO mechanism.[78, 104]

Not long after the discovery of RXFP1 and RXFP2 as RLX receptors by Hsu et al [3], it was shown by Dschietzig et al [105] that RLX can signal through the glucocorticoid receptor (GR) to blunt the secretion of pro-inflammatory cytokines in an RXFP independent manner.[106] They showed that endotoxin stimulated THP-1 monocytes treated with RLX (5-10 $\mathrm{nmol} / \mathrm{L}$ ) secreted significantly less IL-1, IL-6 and TNF $\alpha$, a response that was significantly reduced by the GR antagonist, RU-486. Moreover, since the medium was steroid free, they suggested that RLX interacts with the GR signaling pathway independently of any endogenous glucocorticoid steroids. RLX can blunt the NLRP3 inflammasome formation after ischemia and reperfusion via an endothelial NOS (eNOS) mechanism [107], and blocks neutrophil adhesion in rat coronary endothelial cells in an NO dependent fashion.[99]. Finally, we have shown that RLX can reverse the age associated inflammatory response in aged F-344 Brown/Norway rats through inhibition of various inflammatory pathways and antigen presentation, and that RLX blocks macrophage accumulation in the aged LV, and that these effects are sex-dependent.[8]

\subsection{Relaxin and Heart Failure}

Due to its proven vasodilatory properties, RLX has shown promise in treating patients with heart Failure.[108] A recent review has succinctly outlined the recent clinical trials involving the human recombinant form of RLX, termed Serelaxin [108], and will therefore be considered only briefly here. Early RLX experiments in human trials were interested in safety and tolerability of 
RLX treatment, and Dschietzig et al [16] showed no serious adverse effects of RLX treatment delivered intravenously (IV) for 24 hours in concentrations up to $960 \mu \mathrm{g} / \mathrm{kg} / \mathrm{day}$ in 16 patients, resulting in increased cardiac output, cardiac index, and reduced systemic vascular resistance and NT-pro-BNP. These effects lasted for at least 8 hours post-infusion. A phase II RLX study of more than 230 patients received RLX at 10, 30, 100 and $250 \mu \mathrm{g} / \mathrm{kg} /$ day, IV for 48-hours.[109] The study showed the greatest benefits at $30 \mu \mathrm{g} / \mathrm{kg} /$ day in length of hospital stay, worsening heart failure, dyspnea relief, 60-day readmission and 180-day cardiovascular mortality. Interestingly, in this study, patients receiving $250 \mu \mathrm{g} / \mathrm{kg} /$ day RLX began to show impaired renal function. Based on these studies, a larger phase III trial chose to use $30 \mu \mathrm{g} / \mathrm{kg} / \mathrm{day}$ dosing to study the effects of RLX in acute heart failure in over 1100 heart failure patients.[110] This phase III study showed comparable results seen in phase II trial, though interestingly, showed no benefit in 60-day readmission seen in the phase II trial, but did show reduction in 180-day allcause mortality. Notably, in both the phase II and III trials, RLX led to a drop in systolic blood pressure that led to either stop of treatment or dose readjustment in some patients. Multiple posthoc analyses have been performed to understand which subgroups of patients benefit most from RLX treatment. Metra et al [111] suggests that the greatest benefits are seen in reduced cardiovascular events associated with age ( $>75$ years), lymphocytes at baseline, glomerular filtration rate at baseline, hospital stay in the past year for heart failure and use of beta-blockers. Felker et al [112] showed that RLX had the greatest benefit by reducing cardiovascular deaths not associated with heart failure including reduced sudden cardiac death and stroke. Importantly, Filippatos et al [113] looked at the effects of RLX on the subgroups of heart failure with reduced ejection fraction compared to heart failure with preserved ejection fraction, for which no clinically effective therapy exists. RLX showed no difference in efficacy between the groups on 
improved dyspnea up to 5 days post treatment, heart or renal failure at day 60 and cardiovascular death up to 180 -days.

In contrast to these results, a recent phase III clinical trial in acute heart failure showed no 180-day benefit compared to placebo (www.Novarits.com, March 2017). Much has been made of this failed clinical trial as well as criticism of treating heart failure, a chronic disease, with acute therapies.

McCullough [114] summarizes heart failure clinical trials within the past 15 years with similar treatment durations as the RLX trials and shows that these trials failed to meet their primary endpoints. He contends that a 48-hour treatment is unlikely to resolve phenotypes of heart failure which have been acting to alter organ and tissue substrates for an unspecified amount of time but in the range of years, and that, in contrast to quick hemodynamic improvements from drug therapies, adaptations at the organ and tissue level take longer to reverse, rendering short-term treatments likely to fail in long-term endpoints.

It seems clear that RLX has potential to be a therapy for heart failure patients, yet no studies have been performed to determine the long-term effects of its anti-fibrotic or antiarrhythmic properties, nor have there been studies to determine repeat dosing strategies to obtain the greatest benefit.

\subsection{Relaxin Mimetics}

As detailed above, RLX has shown diverse protective effects on the cardiovascular and other organ systems. However, the full-chain RLX is costly to make, and has normally been associated with a short half-life (0.5-4 hrs) [22] in the blood.[115] Recent work has therefore led 
to the development of RLX mimetics to obtain the beneficial anti-fibrotic characteristics of RLX by more economical means with potentially longer-lasting effects. Once such molecule is comprised only of residues 7-33 of the B-chain of RLX (B7-33).[116] B7-33 was shown to have a suppressed effect on cAMP compared to RLX, but increased phosphorylation of ERK1/2 and expression of MMP-2 in myofibroblasts to a level comparable to RLX. In addition, B7-33 reduced collagen expression due to myocardial infarction or isoproterenol, and decreased lung fibrosis due to ovalbumin. A further study of B7-33 showed the mimetics ability to replicate RLXs effects in mesenteric arteries and to prevent endothelial dysfunction.[117]

The small molecule, ML290, has been described as an RXFP1 agonist, resulting in increased cAMP levels and reduced collagen I gene expression in chronic liver disease.[118] In addition, ML290 has been shown to have similar signaling responses as RLX (e.g. increased cAMP through certain G-coupled proteins, p38MAPK, MMP-2, pSMAD2 and cGMP), while they differed in active p-ERK1/2 levels, though, interestingly, priming with ML290 enhanced RLXs effect on active p-ERK1/2. Moreover, ML290 did not appear to compete with RLX for RXFP1 binding suggesting an allosteric mechanism of action. ML290 appears to be an RXPF1 agonist in humans, monkey's and pigs, and an antagonist in mice, suggesting species bias in ML290 signaling.[118, 119, 120] However, studies with mouse RXFP1 replaced with humanized RXFP1 have shown that animal models can be developed to study the effects of ML290 in smaller rodents.[121] In contrast to RLX, ML290 has been shown to have a half-life in the heart and plasma of $\sim 8$ hours, opening the possibility of longer lasting effects with less treatment time.[119] An apparent limitation of RLX studies is the wide range of reported RLX half-life values in the plasma (reported from 10 minutes to 7-8 hours).[22, 119, 122] Duration or method 
of administration, and whether RLX concentrations reach steady state in the blood may be a determinant and should be monitored closely.

In sum, these data provide compelling evidence that RLX mimetics can re-create many of the positive effects of RLX with quicker production times and lower cost, and thus, while more testing is vital, suggest alternative RLX therapies for myriad of cardiovascular, renal, lung and other organ diseases.

\subsection{Introduction Conclusion}

Relaxin (RLX) is a hormone of pregnancy with a pleiotropy of effects in the heart, vasculature, lung, kidney and liver. RLX increases arterial compliance, reduces vascular resistance and increases cardiac output, with the vasodilatory properties largely mediated via nitric oxide. RLX inhibits pathological collagen expression from fibroblasts in response to pathological stimuli, without effecting basal levels of collagen, and can suppress cardiac arrhythmias through increased Nav1.5 protein expression, sodium current and altered Cx43 phosphorylation and localization. To fully appreciate RLX's pleotropic effects, it is important to note that different actions occur on a different time-scale. For instance, Piedras-Renteria et al [74, 75] showed that RLX reduces ITO within minutes after its addition to atrial muscle. Similarly, Han et al [73] showed that RLX acts acutely on Ca2+ currents in rabbit sinoatrial node pacemaker cells. In contrast, several reports indicated that RLX changed the genomics of cells and tissues to increase the expression of cardiac ion channel protein and to decrease the

expression of collagen; processes that occur over a period of 24-72 hours.[13, 14, 43] Still other actions of RLX such as the reversal of fibrosis require approximately 2-weeks of RLX 
treatment. $[11,13,14]$ These findings point to the need to design a treatment protocol that is suitable to an experimental or clinical objective.

The RELAX-AHF clinical trial tested RLX as a therapy for acute decompensated heart failure at a dose of $30 \mu \mathrm{g} / \mathrm{kg} /$ day for 2-days delivered intravenously.[123] RLX was well tolerated, safe and significantly reduced 180-day mortality.[123] In a subsequent trial (see www.Novartis.com), RLX had no beneficial effects compared to standard of care perhaps due to an inadequate treatment protocol. Little is known regarding the dynamics of endogenous RLX in normal males and non-pregnant females and there is the possibility that the hormone levels are at picomolar or sub-picomolar levels and pulsatile in the blood stream. Future studies should elucidate the molecular mechanisms that stimulate RLX synthesis, the cells that secrete RLX and the normal range of RLX in the blood stream. With a better understanding of RLX biology and the development of long-lasting stable RLX mimetics, this hormone could be central to several new and effective therapies. 


\subsection{Hypotheses}

Based on work by Liang et al [82] suggesting that canonical Wnt signaling could regulate expression of the voltage gated sodium channel, Nav1.5, and its associated current, $\mathrm{I}_{\mathrm{Na}}$, we hypothesized that RLX would inhibit genomic expression of canonical Wnts and block Wnt signaling (see also sections 3.1 and 5.1). Surprisingly, we discovered that RLX activates canonical Wnt signaling, and therefore tested the hypothesis that canonical Wnt signaling was necessary for the increased expression of Nav1.5 in a rat model of aging (see also sections 3.2 and 5.2). Finally, based on RLX's anti-arrhythmic and anti-fibrotic properties, we hypothesized that RLX would be a beneficial therapy in a rat model of pulmonary arterial hypertension, to reduce fibrosis and suppress ventricular arrhythmias (see also sections 3.3 and 5.3). 


\subsection{Specific Aims}

Aim 1. To test the hypothesis that aging altered and relaxin reversed gene expression which could lead to fibrosis and action potential conduction alterations, RNA-sequencing was performed on young and aged rats with or without RLX treatment (see section 5.1).

Aim 2. Based on data from Liang et al [82], Asimaki et al [83, 124] and Chelko et al [84], showing Wnt signaling altered Nav1.5, Cx43 and $\beta$-catenin expression, we tested the hypothesis that RLX modulates Wnt signaling to mediate increased Nav1.5 expression in aged rats (see section 5.2).

Aim 3. Based on RLX's anti-fibrotic and anti-arrhythmic actions, we tested the hypothesis that RLX would be a beneficial therapy to rats with pulmonary arterial hypertension (which suffer from ventricular arrhythmias, asystole and pulmonary and cardiac fibrosis) through improved action potential conduction velocity, suppression of ventricular arrhythmias and reduced cardiovascular and pulmonary fibrosis (see section 5.3). 


\subsection{Methods}

\section{Animal models}

Young (9-month-old) and aged (24-month-old) male and female F-344/Brown Norway rats were obtained from the National Institute of Aging (Chapter 5.1 and 5.2). Rats were treated with vehicle (sodium acetate) or RLX (400 $\mu \mathrm{g} / \mathrm{kg} /$ day) for 14 days via subcutaneous osmotic mini pumps. Left ventricular tissue was snap frozen in liquid nitrogen and stored at -80 degrees Celsius until use. Studies were performed in accordance with the Guide for the Care and Use of Laboratory Animals and were approved by the Institutional Animal Care and Use Committee at the University of Pittsburgh.

A total of 36 male Sprague Dawley rats (Chapter 5.3; mean weight 300 g, Charles Rivers) were included in the study. A subset of animals $(n=27)$ received the VEGFR2 antagonist SU5416 (Sigma). SU5416 was dissolved in CMC buffer (0.5\% sodium carboxymethyl cellulose, $0.4 \%$ polysorbate $80,0.9 \%$ sodium chloride, and $0.9 \%$ benzyl alcohol) and was given as a single $20 \mathrm{mg} / \mathrm{kg}$ subcutaneous injection. The control animals $(\mathrm{n}=9)$ received subcutaneous injection of vehicle $(2 \mathrm{ml} / \mathrm{kg})$. Next, animals were implanted with osmotic mini-pumps (Alzet Cupertino, CA, Model 2ML4) on day zero and were replaced on day 28 to deliver: the vehicle (Na-acetate, $n=11), 30(n=8)$ or $400 \mu \mathrm{g} / \mathrm{kg} /$ day $(\mathrm{n}=11)$ of RLX for 6weeks. The rats were placed in a hypoxic chamber $\left(10 \% \mathrm{O}_{2}\right)$ for 3-weeks followed by 3-weeks in a normoxic environment $\left(21 \% \mathrm{O}_{2}\right)$. Blood samples were taken from each rat at the beginning (day zero) and the end of the experiments (day 42) and tested for levels of RLX using an ELISA assay (ELISA Kit, R\&D 
Systems, DRL200-Human Relaxin-2 Quantikine). Relaxin levels in the blood stream were below detectable levels for control rats at day 42 . At day 42 , RLX levels were $2.6 \pm 1 \mathrm{ng} / \mathrm{mL}$ and $15 \pm 12$ $\mathrm{ng} / \mathrm{mL}$, respectively for rats treated with the low or high-dose of RLX.

All animals received humane care in housing facilities at the University of Pittsburgh in accordance with the Guide for the Care and Use of Laboratory Animals published by the National

Institutes of Health (publication 85-23, revised 1985). The study protocol was approved by the Institutional Animal Care and Use Committee of the University of Pittsburgh. Relaxin was the generous gift Dr. Mario Bigazzi and Dr. Daniele Bani and the Foundation for Research on Relaxin in Cardiovascular and Other Diseases.

RNA-sequencing

Three animals were analyzed from male groups and four animals from female groups (24-month-old \pm RLX, 9-month-old \pm RLX). A total of $1 \mu \mathrm{g}$ of RNA was sent to the University of Pittsburgh Genomics Core for sequencing and library preparation. Library preparation was performed using the TruSEQ Stranded Total RNA Sample Preparation Kit (Illumina, San Diego, CA) according to manufacturer's instructions. Following removal of ribosomal sequences, RNA was fragmented for 8 minutes and reverse transcription performed. Double stranded cDNA was subjected to 3' adenylation and ligation of sequencing adapters. Sequencing was carried out on a NextSeq 500 (Illumina) for $2 \times 75$ bp paired end reads. Loading concentration was 1.4 pM for females and $1.2 \mathrm{pM}$ for males.

RNA-sequencing analysis

Raw transcript data was imported into Biomedical Genomics Workbench 3.0.1 and reads were mapped to the rat reference genome. Differentially expressed genes (DEGs) used in 
pathway analysis were determined between old and young rats with and without RLX using filters to select genes with Reads Per Kilobase of transcript per Million, RPKM 1, absolute fold change (FC) > 1.5 using total gene count and a false discovery rate (FDR) 0.05. Fisher's exact test was used to test for pathway significance. Activation (z-score $>2$, orange bars) or inhibition (z-score < 2, blue bars) of each pathway was determined by Ingenuity Pathway Analysis (IPA) and is a measure of experimentally determined gene expression changes reported in the literature. For reporting of individual genes, data are presented as FC with pvalue and FDR. DEGs filtered for pathway analysis were imported into Ingenuity Pathway Analysis and MetaCore to determine signaling pathways and upstream regulators affected by aging or RLX treatment. Aging studies were carried out by comparing RNA-seq data from 24month old untreated (UNT) vs. 9-month old UNT rats. RLXs effect was measured by comparing 24-month-old + RLX vs. 24-month-old UNT rats. The data from this publication have been deposited in NCBI's Gene Expression Omnibus (GEO), and are accessible via GEO series number GSE106377 at https://www.ncbi.nlm.nih.gov/geo/query/acc.cgi?acc=GSE106377.

\section{Immunofluorescence}

Male ( $n=3-4 /$ group) and Female ( $n=5 /$ group) left ventricular tissue were thawed and fixed in 2\% PFA at room temperature and left in $30 \%$ sucrose for 24 hours at 4 degrees Celsius. LV tissue sections $(7 \mu \mathrm{m})$ were placed in $0.1 \%$ Triton $100-\mathrm{x}$ for 10 minutes. Sections were blocked with 2\% BSA for 30 minutes followed by a 90-minute incubation with primary antibodies, rabbit-anti-F4/80 polyclonal (Thermo Fischer, PA5-21399), mouse-anti-CD4 monoclonal (BioRad, MCA55GA), and mouse-anti ANP monoclonal (Santa Cruz Biotechnology, sc515701), Guinea Pig and Rabbit anti-Nav1.5 (AGP-008, Alomone Labs, 1:200 
in tissue and ab56240, Abcam, 1:200 in cells), rabbit anti- $\beta$-catenin (ab32572, Abcam, 1:250), mouse anti-Cx43 (sc-13558, Santa Cruz Biotechnology, Inc, 1:100), rabbit anti-collagen I (ab34710, Abcam, 1:200), mouse anti-Wnt1 (10C8, Thermo Fisher, 1:200). Sections were washed 3x 5minutes and incubated with secondary antibodies for $45 \mathrm{~min}$ and another wash. Finally, sections were stained for 1 minute with DAPI and coverslips mounted with gelvatol. Images were taken on an Olympus Fluoview inverted confocal microscope at 60x magnification. Image parameters were obtained for one image and kept constant throughout imaging. Images were analyzed using ImageJ software and only circular staining with a clear association with a nucleus were included in the macrophage and T-cell analysis. ANOVA was used to determine statistically significant differences between groups with Tukey's post hoc analysis. Data is presented as mean \pm SEM. $\mathrm{P}<0.05$ was considered statistically significant.

\section{Plasma relaxin concentration:}

Circulating levels of RLX in the aged rats were determined using a pre-packaged Quantikine ELISA kit (R\&D Systems, DRL200) according to manufacturer instructions.

\section{Optical Mapping:}

The optical mapping apparatus was described in detail in several reports $[125,126]$. Briefly, hearts were excised and perfused on a Langendorff apparatus with Tyrode's solution containing (in mM): $\mathrm{NaCl}$ (130), $\mathrm{KCl}$ (4.5), KH2PO4 (0.6), Na2HP04 (0.6), MgSO4 (1.2), HEPES (10), NaHCO3 (24), Glucose (50), gassed with 95\% O2 and 5\% CO2, pH 7.0 at 37 oC. Hearts were placed in a custom-designed chamber to abate motion artifacts and blebbistatin (5-7 $\mu \mathrm{M})$ briefly added to the perfusate to minimize movement artifacts. Bolus injections of voltage (RH $23750 \mu \mathrm{l}$ of $1 \mathrm{mg} / \mathrm{mL}$ dimethyl sulfoxide (DMSO)) and Ca2+-indicator dye (Rhod-2/AM, $80 \mu \mathrm{l}$ of $1 \mathrm{mg} / \mathrm{mL}$ DMSO) were made in the air-trap above the aortic cannula. Fluorescence from 
the epicardium was collected with a camera lens, split with a $570 \mathrm{~nm}$ dichroic mirror and focused on two CMOS cameras (Sci-Media UltimaOne) capturing at the fluorescence emission at 570$595 \mathrm{~nm}$ for cytosolic $\mathrm{Ca} 2+$ and $610-750 \mathrm{~nm}$ wavelengths for voltage. The hearts were paced at various cycle lengths (CL) and with programmed stimulation, starting with a baseline CL for 10 beats at $\mathrm{S} 1-\mathrm{S} 1=250 \mathrm{~ms}$ followed by a single $\mathrm{S} 2$ pulse delivered at decreasing $\mathrm{S} 1-\mathrm{S} 2$ intervals. Pacing protocols were used to measure CV at various stable CLs (S1-S1) and CV restitution kinetics was measured through the $\mathrm{CV}$ of the premature impulse delivered at decreasing $\mathrm{S} 1-\mathrm{S} 2$, until the premature impulse failed to capture or triggered an arrhythmia.

\section{Immunohistochemistry:}

Tissue sections $(7 \mu \mathrm{m})$ were treated with $0.1 \%$ Triton $\mathrm{X}-100$ followed by block with $2 \%$ BSA. Primary antibody was added for 1 hour at room temperature. antibodies were used to measure protein expression. Secondary antibodies were applied for 1 hour at room temperature. Immunofluorescence imaging was performed using an Olympus Fluoview inverted confocal microscope or an Olympus Provis light microscope. Isolated cardiomyocytes and fibroblasts were stained in a similar manner after fixation with $4 \%$ paraformaldehyde. ImageJ was used for image analysis.

\section{Myocyte Isolation:}

Excised hearts were placed on a Langendorff apparatus and perfused for 4 minutes with Perfusion Buffer containing (in $\mathrm{mM}$ ): $\mathrm{NaCl}$ (130), $\mathrm{KCl}$ (14.7), $\mathrm{KH} 2 \mathrm{PO} 4$ (0.6), Na2HP04 (0.6), MgSO4 (1.2), HEPES (10), NaHCO3 (4.6), Taurine (30), BDM (10), Glucose (5.5), pH 7.0. Heart was perfused for 11-14 minutes with digestion buffer containing: $50 \mathrm{~mL}$ Perfusion Buffer with $2 \mathrm{mg} / \mathrm{mL}$ Collagenase Type II. Heart was placed in small glass beaker with $3 \mathrm{~mL}$ digestion buffer and minced into small pieces with surgical scissors. Additional mincing was conducted by 
cutting plastic transfer pipettes at 45 degrees at largest diameter, slowing reducing pipette diameter size as tissue becomes solubilized. Stopping buffer $(10 \mathrm{~mL})$ was added containing: 45 mL Perfusion Buffer, $5 \mathrm{~mL}$ of $10 \%$ FBS and $12.5 \mu \mathrm{M} \mathrm{CaCl2}$. Myocytes were transferred to a 50 $\mathrm{mL}$ tube through a cell strainer primed with stopping buffer and allowed to pellet ( 20 minutes). Myocytes were washed with Calcium re-introduction solutions $(100 \mu \mathrm{M}, 400 \mu \mathrm{M}$ and $900 \mu \mathrm{M}$ : diluted in stopping buffer) with pelleting allowed between washes. Cells were mixed with plating medium containing: 10\% FBS, Blebbistatin $(25 \mu \mathrm{M})$, HEPEPS $(10 \mathrm{mM})$, ATP $(2 \mathrm{mM})$ and PrimocinTM (diluted $50 \mathrm{mg}$ to $500 \mathrm{~mL}$ MEM, Invivogen, San Diego, CA. USA). Cells were placed on laminin coated coverslips, in 24 well plates and incubated at 37 oC for 2 hours. Plating medium was replaced by culture medium containing: $0.1 \%$ BSA, Blebbistatin (25 uM), HEPES (10 mM), ITS (250 uL of 100x stock) and PrimocinTM.

\section{Picro-Sirius Red Stain:}

Left ventricular sections $(7 \mu \mathrm{m})$ were washed in Xylene followed by washes in $100 \%$ and 95\% EtOH. Tissue was placed in Hematoxylin, followed by tap water wash and Picro-Sirius Red application. This was followed by washes with acidic water, $100 \% \mathrm{EtOH}$ and Xylene. Finally, coverslips were mounted onto slides with Permount. Imaging was performed using an Olympus Provis Light Microscope at 10x magnification. Data is reported as collagen:tissue ratio.

\section{Fibroblast Culture:}

Primary cardiac fibroblasts isolated from the male F-344 rat model were grown to 80$90 \%$ confluence and washed with HEPES BSS $(100 \mu \mathrm{L} / \mathrm{cm} 2)$, followed by Trypsin/EDTA $(100 \mu \mathrm{L} / \mathrm{cm} 2)$ for less than 5 minutes, and Trypsin Neutralization Solution $(100 \mu \mathrm{L} / \mathrm{cm} 2)$. Solution was then spun down for 3 minutes at $220 \mathrm{x}$ g followed by resuspension in pre-warmed growth media. Cells were counted and stored in Cryo-SFM media (PromoCell, C-29910) at $-80{ }^{\circ} \mathrm{C}$ for 
slow freezing for 24 hours and moved to $-120{ }^{\circ} \mathrm{C}$ for long term storage. Frozen fibroblasts were thawed at $37^{\circ} \mathrm{C}$ until no ice remained in the vial ( $\sim 2$ minutes). Fibroblasts were seeded between 5,000-10,000 cell/cm2. Media (PromoCell, C-23130) was replaced after 24 hours and every 2 days thereafter until $90 \%$ confluency was reached. Fibroblasts were treated with RLX (25 nM), TGF $\beta(2 \mathrm{ng} / \mathrm{mL}), \operatorname{Dkk}(0.1 \mu \mathrm{g} / \mathrm{mL}), \mathrm{Wnt} 1(0.1 \mu \mathrm{g} / \mathrm{mL})$ and Wnt3a $(0.1 \mu \mathrm{g} / \mathrm{mL})$.

\section{RT-PCR Analysis}

RNA was isolated (RNAEasy, Qiagen) and copied to cDNA (High Capacity Reverse Transcription kit, Applied Biosystems) according to manufacturer protocols. A Syber-greenbased formulation (Absolute Sybr-Green, Thermo Fischer Scientific, Waltham, MA) was utilized for fluorescence-based kinetic real-time PCR using an Applied Biosystems model 7000 detection system (Applied Biosystems Inc., Foster City, CA). Expression levels of RNAs of interest were normalized to that of GAPDH using the $\triangle \Delta \mathrm{Ct}$ method, and reported relative to the mean of the WTV group. Primer pair sequences (forward and reverse for each target, listed 5' to 3') used for RT-PCR are as follows:

MMP-2: gcaccaccgaggattatgac, cacccacagtggacatagca;

MMP-9: cctctgcatgaagacgacataa, ggtcaggtttagagccacga;

aSMA: tggctgatggagtact-tc, gatagagaagccaggatg;

Wnt1: cctgcacctgcgactacag, ggttcatgaggaagcgtagg

GAPDH: agctggtcatcaatgggaa, atttgatgttagcgggatc.

\section{Western Blot}

Protein from aged rat lysates \pm RLX ( $n=3$ /group) was separated using pre-cast MiniPROTEAN TGX 7.5\% polyacrylamide gels and transferred to a PVDF membrane. Membranes were then probed for mouse anti-DKK1 (1:500, Santa Cruz sc374574) and rabbit anti- $\alpha$-tubulin 
(1:1000, Abcam, ab4047). Membranes were developed using Pierce ECL Western Blotting Substrate (ThermoScientific, \#32109) according to manufacturer instructions and developed by autoradiography. Quantification of the optical density of DKK1 or tubulin bands were performed using ImageJ software and DKK1 expression was normalized to $\alpha$-tubulin.

\section{Right ventricular pressure and Doppler Flow Measurements}

Right ventricular hemodynamics were assessed from simultaneously collected Doppler flow (DSPW, Indus Instruments, Webster, TX) and direct right and left ventricular pressurevolume measurements (Transonic, Scisence and IOX2, EMKA). Briefly, rats were anesthetized using isoflurane and intubated before opening the thorax to expose the heart and lungs. The admittance pressurevolume catheter tip was inserted into the RV to measure RV pressure. Pulmonary artery Doppler flow velocities were simultaneously measured as PA pressure and RV pressure. All signals were stored, and post-process analysis was performed using a combination of commercial analysis software and custom Matlab programs (R2014a, MathWorks, MA).

Pulmonary artery Doppler flow velocity envelopes were traced and used to calculate mean velocity. Stroke distance was determined from the integral of the velocity envelope and converted to stroke volume assuming a $2.5 \mathrm{~mm}$ diameter of the pulmonary artery (stroke volume $=$ stroke distance $\mathrm{x}$ cross sectional area). Cardiac output was calculated as the product of stroke volume and heart rate.

\section{Programmed Stimulation}

Arrhythmia vulnerability was tested by pacing each heart from the LV or RV using a programmed stimulation protocol consisting of 10-20 S1 pulses at 250 ms cycle length (CL) followed by a premature S2 pulse with progressively shorter S1-S2 interval steps: from CLs of 250 to $100 \mathrm{~ms}, \mathrm{~S} 1-\mathrm{S} 2$ decreased in $20 \mathrm{~ms}$ steps; from 100 to $60 \mathrm{~ms}$ in $10 \mathrm{~ms}$ steps and 60 to $35 \mathrm{~ms}$ 
in 5ms steps, until loss of capture or the initiation of an arrhythmia. APD Restitution Kinetics (RK) curves were generated by plotting mean $\mathrm{APD}_{75}$ (from a minimum of 100 pixels on $\mathrm{RV}$ or LV versus S1-S2 interval in milliseconds. CV RK curves were generated by plotting the mean CV vs. S1-S2 interval in milliseconds. Transient ventricular arrhythmia lasted 5-15 seconds and self-terminated, whereas sustained ventricular arrhythmia lasted $>3$ minutes and could be terminated with a bolus injection of $1 \mathrm{M} \mathrm{KCl}$.

\section{$R V$ hypertrophy: Fulton Index and RV/Tibia ratio}

$\mathrm{RV}$ weights normalized to tibia length were used as indexes of RV mass. Fulton index (weight of RV/weight of LV + septum) was used as an index of RV hypertrophy.

\section{Histology (PAH rats)}

$\mathrm{RV}$ and LV tissues ( $\mathrm{n}>4$ /group) were fixed in $2 \%$ PFA, equilibrated in $30 \%$ sucrose and flash frozen. Frozen sections (5- $\mu \mathrm{m})$ were stained with Mason's Trichrome and imaged with an Olympus Provis AX70 microscope at 10x magnification. Four random images were taken from each tissue section for analysis. Once optimal imaging parameters were determined, all subsequent images were obtained using the same conditions. Analysis was performed using ImageJ software. Collagen I to tissue area ratio was calculated as previously described.[23] Comparison of groups to control or SuHx was done using ANOVA in GraphPad Prism, and $\mathrm{p}<0.05$ was considered statistically significant.

The right bronchus was ligated, and the right lung was stored at $-80^{\circ} \mathrm{C}$. The left lung was inflated and fixed with $10 \%$ buffered formalin at a height of $25 \mathrm{~cm}$ and immersed in formalin for at least $72 \mathrm{~h}$ before being embedded. Serial tissue sections $(5-\mu \mathrm{m})$ from paraffin-embedded lungs were deparaffinized and stained with hematoxylin and eosin (H\&E), Mason's trichrome, and Verhoeff's Van Gieson (VVG) for assessment of occlusive and plexiform lesions and 
perivascular fibrosis. Lungs from control, SuHx, low-dose (RLX LD), and high dose (RLX HD) $(\mathrm{n}=4$ per group) rats were embedded in paraffin, sectioned $(5-\mu \mathrm{m})$ and stained with Mason's Trichrome. Collagen Images were taken with an Olympus Provis AX70 microscope at 10x magnification so that the entire surface area of each tissue was imaged. Fibrosis was analyzed as the collagen-to-tissue ratio using ImageJ software. Airspaces in the tissue were excluded in the analysis. The statistical significance of average collagen difference between groups was evaluated using ANOVA in GraphPad Prism. $\mathrm{P}<0.05$ was considered statistically significant. Pulmonary media vascular remodeling was assessed by staining pulmonary arterial wall smooth muscle cells with a mouse monoclonal and anti-smooth muscle-alpha actin antibody at a dilution of 1/100 (Laboratory Vision, Fremont, CA). The Aperio ScanScope XT system (Aperio Inc, Vista, CA, USA) was used to scan entire glass slides at 20x or 40x magnification and Aperio ImageScope software was used to analyze digital slides. On average, between 70-110 small size pulmonary arteries $(<50 \mu \mathrm{M})$ per animal were analyzed for pulmonary vascular remodeling (neomuscularization), incidence of occlusive vascular lesion, and presence of plexiform lesions (PLX) and expressed as number of PLX per slide or normalized by number of examined small size arteries.

\section{Statistical Analysis:}

Comparisons between two groups were done using an unpaired, 2-tailed t-test, or the Mann-Whitney $\mathrm{U}$ test. Comparisons of three or more groups were done using ANOVA or Kruskal-Wallis tests. Nuclear $\beta$-catenin was compared using Chi-squared. All data is presented as mean \pm SEM, unless otherwise noted. Statistical comparisons were performed using Graphpad Prism software. A value of $\mathrm{P}<0.05$ was considered to be statistically significant. 


\subsection{Results}

In order to more fully understand the long-term beneficial effects of RLX in HF, AF and fibrosis, as described in Chapter 1, we investigated the underlying effects of RLX on gene expression using RNA-sequencing in our aged rat model of disease.

Aging is a major risk factor for atrial fibrillation (AF) [127] and heart failure (HF), [1료 $]$ which may reach pandemic proportions due to an increasingly aging population, estimated to reach 70 million people over the age of 65 in the United States by 2030.[129] HF is defined as the inability of the heart muscle to pump enough blood to meet the energy demands of the body [13], and aging is associated with multiple maladaptive vascular and cardiac structural, electrical and functional changes that increase the susceptibly to $\mathrm{HF}, \mathrm{AF}$ and other cardiovascular diseases (CDs). [13, 129] Structural remodeling in aging includes: increases in vascular stiffness, left ventricular (LV) and aortic wall thickness [129], fibrosis [131] and myocyte hypertrophy that promote diastolic dysfunction, coronary heart disease, [129] AF [132] and HF. [131] In sum, age-related maladaptive responses lower the threshold for CD development. [131] Recent evidence posits that aging brings-on the gradual development of a chronic, low-grade inflammation, termed "inflammaging" which is possibly the greatest risk factor for many agerelated diseases. [133] Chronic inflammation can result from failed resolution of an injury by the body or stimuli resulting in asymptomatic responses.[134] Inflammation has been linked to multiple CDs including myocardial infarction, resulting in increased leukocyte infiltration that 
leads to pathological left ventricular (LV) remodeling.[135] In addition, complement cascade activity can be activated in the presence of pathogens or tissue injury and has been linked to higher hospitalizations and mortality in ischemia/reperfusion studies, [134, 136] and to inflammation associated with insulin resistance and type II diabetes. [137] Interleukin (IL)-1 and IL-6 have been linked to cardiac hypertrophy, reduced contractility, increased arrhythmia susceptibility, increased C-reactive protein, coronary artery disease [138] and raise stroke risk, while tumor necrosis factor alpha $(\mathrm{TNF} \alpha)$ is associated with LV dilatation and dysfunction and HF progression. [139-141] In addition to inflammatory markers of disease, multiple markers are used clinically to determine HF progression and prognosis. Brain natriuretic peptide (BNP, gene: $N P P B)$ is a marker of $\mathrm{HF}$, is indicative of hemodynamic wall stress and is a risk factor for AF and HF. [140, 142, 143] Like BNP, atrial natriuretic peptide (ANP, gene: NPPA) is a marker of congestive HF [144] and growth differentiation factor (GDF)-15 is implicated in HF and inflammatory and oxidative stress. $[\underline{143}, \underline{145}]$ Clinical trials that suppressed the inflammatoryrelated cytokine, TNF $\alpha$, have yielded disappointing results in chronic HF patients. TNF $\alpha$ chelation had no effect on primary endpoints of hospitalization and death, leading investigators to hypothesize a large degree of redundancy in the immune response related to HF, so that targeting a single cytokine may prove to be ineffective. [136] Studies in patients with rheumatoid arthritis using tocilizumab to block IL-6 signaling through the IL-6 receptor have shown beneficial effects, though resulted in side-effects including pro-atherogenic dyslipidemia and increased low density lipoprotein (LDL), [138] which is associated with increased risk of coronary artery disease. [146]

Although age is a major risk factor for inflammation, AF and HF, $[\underline{133}, \underline{147}, \underline{148}]$ with few exceptions [148-150] there is a noticeable paucity of genetic studies on how global gene 
expression is altered in normal, 'healthy' cardiac aging. Many were done in mice despite the challenge of comparing age-related gene expression patterns in mice and man. Yet, differences in mice may be due to a non-uniform rate of aging in mice.[149] since other studies identified aging gene expression signatures that are conserved in mice, rats and humans, namely, inflammation and the immune response. [148]. This suggests that genomic studies of inflammation in rodents may be relevant to the development of therapies for disorders related to age and inflammation. Furthermore, the challenges of using mouse models of aging are largely dispelled through the use of the F-344 Brown/Norway rat model from the National Institute of Aging, which has been developed and used extensively to study the effects of aging due to its homogeneous and linear rate of aging, increased cardiac collagen production and increased susceptibility to atrial fibrillation and systolic and diastolic dysfunction; all hallmarks of human aging. $[132, \underline{151}, \underline{152}]$

Relaxin (RLX), a hormone of pregnancy, has shown significant cardiovascular benefits including a significant reduction in AF susceptibility in aged [13] and hypertensive [14] rats, primarily through a reversal of fibrosis and an increase in voltage gated sodium channels, Nav1.5, and its current, $\mathrm{I}_{\text {Na. }}$ In phase II clinical trials, RLX reduced 180-day cardiovascular and all-cause mortality in acute decompensated HF patients after 48-hour RLX infusion; [110, 112] however, recent Phase III clinical trials failed to meet the primary endpoint of reduced mortality in HF patients (https://www.novartis.com/news release, March 2017), though details are yet to be published. In spite of these results, RLX has been shown to be a potent anti-fibrotic through regulation of fibroblast activation, collagen secretion and reductions in pro-fibrotic transcripts, $[13, \underline{43}]$ a suppressor of AF through a combination of structural and electrical cardiac remodeling, $[13,14]$ and to increase vascular compliance and reduce systemic vascular 
resistance.[24, 43] In addition, we report here RLX's anti-inflammatory actions, suggesting that RLX acts on multiple signaling pathways and may be a therapy for various diseases associated with these pathological conditions. Despite growing evidence of RLX's beneficial effects, [4, 11, $\underline{13}, \underline{14}, \underline{153]}$ the mechanisms underlying these effects remain unclear. In RELAX-AHF trials, RLX serum concentrations rose rapidly, levelled off after 4 hours of infusion, and decreased quickly post-infusion.[108] The short lifespan of RLX ( 2hrs) suggests that the long-term benefits of RLX stem from long-lasting genomic effects rather than an improvement of hemodynamics at the time of infusion.[110] Here, we test the hypothesis that RLX acts at the genomic level to modify HF and AF related genes and signaling pathways that are activated or inhibited in 'healthy/normal' aging. We show that aging significantly increases gene transcription of multiple pro-inflammatory and HF related genes and signaling pathways. Significantly, we show that RLX reverses the effects of aging in both genders through genetic regulation of these pathways and that aging increases macrophage accumulation in females, but not male left ventricles, which is reversed by RLX treatment. These data provide evidence in support of RLX as a therapy for a plethora of inflammatory diseases through its regulation of multiple cytokines and signaling pathways. 


\subsection{Relaxin Reverses Cardiac Inflammation}

\section{Effects of aging and relaxin on biomarkers of AF and HF}

In the clinical setting, several markers have been associated with the severity and/or mortality of AF and HF patients. In a proteomics study of AF, Lind et al [140] reported an association of multiple proteins with AF using two cohorts. The primary cohort included both genders and a validation cohort included only males. It is interesting to note that they reported thirteen $\mathrm{AF}$ associated proteins in the mixed gender cohort, but merely 5 in the male only cohort, suggesting marked gender differences. Table 2 lists age and RLX-dependent transcription changes of multiple markers thought to be involved in AF and HF development. [139-143, 154157] RNA-sequencing (RNA-seq) analysis showed that aging and aging plus RLX-treatment altered with high significance (FDR $<0.05)$ more than 7 genes associated with $\mathrm{HF}$ and AF in the clinical setting. In Table 2, these genes are highlighted in bold and dark shading and genes exhibiting highly significant changes have double-check marks. In females, aging or RLX altered atrial natriuretic peptide (NPPA), interferon gamma (INFG) and the LDL receptor (LDLR). In males, aging or RLX altered IL-1 $\beta$, IL-6, NPPB, and GDF-15. In addition, several genes exhibited $>1.5$ absolute fold changes with $\mathrm{p}<0.05$, but do not reach the more stringent criterion of FDR $<0.05$ and are labeled with a single check mark. RLX significantly reversed 
multiple transcripts associated with $\mathrm{HF}$ and $\mathrm{AF}$ and therefore appears to not only stop the progression but to reverses the pathological expression of many cytokines.

Atrial natriuretic peptide (ANP), the most differentially expressed gene in female rats, was selected for further protein expression analysis to confirm that the effects of RLX on gene expression had functional consequences. In female rats, 9-month-old controls (Fig 2A) exhibited significantly less ANP than in aged ventricles (Fig 2B). RLX treatment (Fig 2C) significantly reversed aging's effect on ANP expression. In male rats, there was no significant change in ANP expression in the aged versus the young controls ( $\underline{\text { Fig } 2 \mathrm{E}, 2 \mathrm{~F} \text { and } 2 \mathrm{H}})$, though there was a reduction in ANP with RLX treatment ( $\underline{\text { Fig } 2 \mathrm{G} \text { and } 2 \mathrm{H}})$, that did not reach statistical significance. These data match closely the gene expression data in Table 2 (NPPA) for ANP expression.

Upstream analysis (Table 3) searches for differentially expressed genes (DEGs) to predict activation or inhibition of transcription regulators and to determine which molecules can explain gene expression alterations observed in the data sets. Observed DEGs are compared to a database of known interactions with transcriptional regulators kept by Ingenuity, with transcripts grouped together under common regulators.

The quantity of genes controlled by a given transcriptional regulator and the agreement of direction (+/-) of fold change of each DEG to that reported in the literature, leads to a prediction of activation or inhibition of those transcriptional regulators. 
Table 2. RNA-Sequencing Analysis of Cardiovascular Disease Related Genes in Aging. FDR: False Discovery Rate. Multiple test correction technique used in RNA-sequencing data analysis. Underscores indicated gene isoform. Bold: at least one group shows FDR < 0.05 . ns: not significant. Yellow, $\checkmark \checkmark$ : FDR $<0.05$. Blue,$\sqrt{ }$ : Fold Change $>1.5, \mathrm{p}<0.05$.

\begin{tabular}{|c|c|c|c|c|}
\hline \multirow[b]{2}{*}{ Gene } & \multicolumn{2}{|c|}{$\begin{array}{c}\text { Female } \\
\text { Fold Change (p-value, FDR) }\end{array}$} & \multicolumn{2}{|c|}{$\begin{array}{c}\text { Male } \\
\text { Fold Change (p-value, FDR) }\end{array}$} \\
\hline & Aging & RLX & Aging & RLX \\
\hline IL-1及 & $-1.24(\mathrm{~ns})$ & $1.48(\mathrm{~ns})$ & $-1.69(0.02, \mathrm{~ns})$ & $-3.54(<0.0001,<0.01) \checkmark \checkmark$ \\
\hline IL-6 & $1.82(0.03, \mathrm{~ns}) \checkmark$ & $-1.81(0.04, \mathrm{~ns}) \checkmark$ & $1.15(\mathrm{~ns})$ & $-4.35(<0.0001,0.01) \checkmark \checkmark$ \\
\hline IL-6RA & $1.09(\mathrm{~ns})$ & $-1.20(\mathrm{~ns})$ & $-1.10(\mathrm{~ns})$ & $1.21(\mathrm{~ns})$ \\
\hline CRP & Undetected & Undetected & Undetected & Undetected \\
\hline $\mathrm{TNF} \alpha$ & $-1.63(\mathrm{~ns}) \checkmark$ & $2.67(<0.01, \mathrm{~ns}) \checkmark$ & $-1.87(<0.01, \mathrm{~ns}) \checkmark$ & $-1.82(0.03, \mathrm{~ns}) \checkmark$ \\
\hline NPPB & $1.00(\mathrm{~ns})$ & $1.28(\mathrm{~ns})$ & $1.43(0.04, \mathrm{~ns}) \checkmark$ & $-1.84(<0.0001,0.01) \checkmark \checkmark$ \\
\hline NPPA & $10.48(<0.0001,<0.0001) \checkmark \checkmark$ & $-2.95(0.01, \mathrm{~ns}) \checkmark$ & $1.37(\mathrm{~ns})$ & $-1.36(\mathrm{~ns})$ \\
\hline GDF-15 & $-1.45(0.07, \mathrm{~ns})$ & $1.37(\mathrm{~ns})$ & $1.40(\mathrm{~ns})$ & $-3.58(<0.0001,0.03) \checkmark \checkmark$ \\
\hline SPP1 & $2.64(<0.01, \mathrm{~ns}) \checkmark$ & $-2.24(<0.01, \mathrm{~ns}) \checkmark$ & $-1.10(\mathrm{~ns})$ & $-3.42(<0.01, \mathrm{~ns}) \checkmark$ \\
\hline CST3 & $1.35(0.01, \mathrm{~ns})$ & $-1.47(0.04, \mathrm{~ns})$ & $1.02(\mathrm{~ns})$ & $-1.46(<0.01, \mathrm{~ns})$ \\
\hline IFNG & $6.10(<0.0001,<0.01) \checkmark \checkmark$ & $-3.25(<0.01, \mathrm{~ns}) \checkmark$ & $-2.48(0.03, \mathrm{~ns}) \checkmark$ & $1.09(\mathrm{~ns})$ \\
\hline FGF-23 & $1.41(\mathrm{~ns})$ & $-1.14(\mathrm{~ns})$ & $-4.44(<0.01, \mathrm{~ns}) \checkmark$ & $1.28(\mathrm{~ns})$ \\
\hline FABP4 & $1.25(\mathrm{~ns})$ & $-1.09(\mathrm{~ns})$ & $1.81(<0.01, \mathrm{~ns}) \checkmark$ & $-1.03(\mathrm{~ns})$ \\
\hline CSTB & $1.28(0.02, \mathrm{~ns})$ & $-1.18(\mathrm{~ns})$ & $1.39(\mathrm{~ns})$ & $-1.47(\mathrm{~ns})$ \\
\hline IL-1RA & $1.78(<0.01, \mathrm{~ns}) \checkmark$ & $-1.32(\mathrm{~ns})$ & $2.11(0.04, \mathrm{~ns}) \checkmark$ & $-2.12(0.03, \mathrm{~ns}) \checkmark$ \\
\hline TNF-R1 & $-1.34(0.04, \mathrm{~ns})$ & $1.14(\mathrm{~ns})$ & $-1.16(\mathrm{~ns})$ & $-1.16(\mathrm{~ns})$ \\
\hline TNF-R2 & $1.05(\mathrm{~ns})$ & $-1.17(\mathrm{~ns})$ & $-1.19(\mathrm{~ns})$ & $1.18(\mathrm{~ns})$ \\
\hline Plaur_1 & $1.2(\mathrm{~ns})$ & $-1.6(0.02, \mathrm{~ns}) \checkmark$ & $-1.21(\mathrm{~ns})$ & $-1.39(\mathrm{~ns})$ \\
\hline Plaur_2 & $1.77(\mathrm{~ns})$ & $-1.76(\mathrm{~ns})$ & $1.85(\mathrm{~ns})$ & $-5.56(<0.01, \mathrm{~ns}) \checkmark$ \\
\hline Plaur_3 & $2.65(0.01, \mathrm{~ns}) \checkmark$ & $-3.55(<0.01, \mathrm{~ns}) \checkmark$ & $-1.2(\mathrm{~ns})$ & $-5.29(0.01, \mathrm{~ns}) \checkmark$ \\
\hline CSF-1 & $1.4(<0.01, \mathrm{~ns})$ & $-1.18(\mathrm{~ns})$ & $1.23(\mathrm{~ns})$ & $1.21(\mathrm{~ns})$ \\
\hline $\mathrm{AM}$ & $1.42(0.01, \mathrm{~ns})$ & $-1.25(\mathrm{~ns})$ & $1.06(\mathrm{~ns})$ & $-1.14(\mathrm{~ns})$ \\
\hline TNNT2_1 & $-1.05(\mathrm{~ns})$ & $1.21(\mathrm{~ns})$ & $1.3(\mathrm{~ns})$ & $-1.53(<0.01, \mathrm{~ns}) \checkmark$ \\
\hline TNNT2_2 & $-1.13(\mathrm{~ns})$ & $1.2(\mathrm{~ns})$ & $1.26(\mathrm{~ns})$ & $-1.26(\mathrm{~ns})$ \\
\hline LDLR & $-1.91(<0.01,0.05) \checkmark \checkmark$ & $1.56(0.01, \mathrm{~ns}) \checkmark$ & $-2.52(<0.01, \mathrm{~ns}) \checkmark$ & $1.32(\mathrm{~ns})$ \\
\hline
\end{tabular}



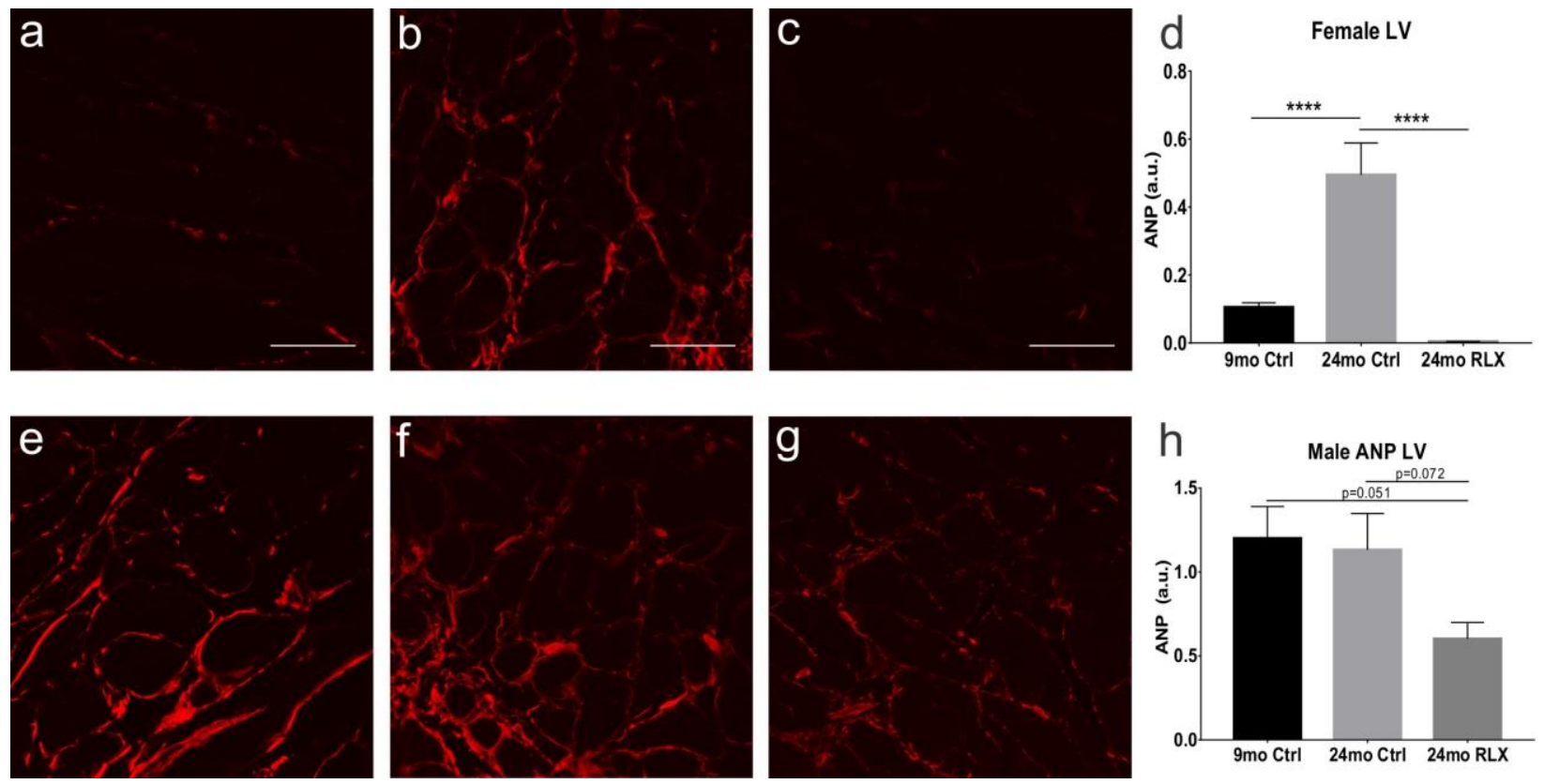

Fig 2. Protein Expression of ANP in Female and Male Hearts. A-D. Female. Female aging (B) resulted in upregulation of ANP expression compared to young controls (A) and was reversed by RLX (C). D. Quantification of ANP expression in female LV. E-H. Male. In male rats, aging (F) did not increase ANP expression compared to young controls (E), though there was a reduction in ANP with RLX treatment (G) though this did not reach significance compared to young or aged males. H. Quantification of ANP expression in male LV. Red: ANP. : p <0.0001. Scale bar: $50 \mu \mathrm{m}$

Table 3. Upstream Analysis of RNA-sequencing Gene Expression Data

\begin{tabular}{|c|c|c|c|c|}
\hline \multirow[b]{2}{*}{ Upstream regulator } & \multicolumn{2}{|c|}{ Female } & \multicolumn{2}{|c|}{ Male } \\
\hline & Aging & RLX & Aging & RLX \\
\hline Hydrogen Peroxide & & Inhibited & & Inhibited \\
\hline NFkB (complex) & Activated & Inhibited & & Inhibited \\
\hline TLR4 & Activated & & & Inhibited \\
\hline IFNG & Activated & Inhibited & & Inhibited \\
\hline IL6 & Activated & Inhibited & & Inhibited \\
\hline LPS & Activated & Inhibited & & Inhibited \\
\hline Sirolimus & Inhibited & & & Activated \\
\hline TGF $\beta 1$ & Activated & Inhibited & & Inhibited \\
\hline SMAD7 & Inhibited & Activated & Activated & \\
\hline
\end{tabular}


Table 4. Chemokine and Chemokine Receptor Transcripts. FDR: False Discovery Rate. Multiple test correction technique used in RNAsequencing data analysis. Bold: at least one group shows FDR $<0.05$. NS: not significant. Yellow, $\checkmark \checkmark$ : FDR $<0.05$. Blue, $\sqrt{ }: \mathrm{p}<0.05$.

\begin{tabular}{|c|c|c|c|c|}
\hline \multirow[b]{2}{*}{ Gene } & \multicolumn{2}{|c|}{$\begin{array}{c}\text { Female } \\
\text { Fold change (p-value, FDR) }\end{array}$} & \multicolumn{2}{|c|}{$\begin{array}{c}\text { Male } \\
\text { Fold change (p-value, FDR) }\end{array}$} \\
\hline & Aging & RLX & Aging & RLX \\
\hline CCL1 & $-1.02(\mathrm{~ns})$ & $1.46(\mathrm{~ns})$ & $-3.64(<0.0001,0.03) \checkmark \checkmark$ & $-1.21(\mathrm{~ns})$ \\
\hline CCL2 & $1.23(\mathrm{~ns})$ & $1.23(\mathrm{~ns})$ & $1.43(\mathrm{~ns})$ & $-3.26(<0.0001,0.04) \checkmark \checkmark$ \\
\hline CCL3 & $1.23(\mathrm{~ns})$ & $-2.62(<0.01, \mathrm{~ns}) \checkmark$ & $-1.15(\mathrm{~ns})$ & $1.89(0.02, \mathrm{~ns}) \checkmark$ \\
\hline CCL5 & $1.79(<0.0001,<0.001) \checkmark \checkmark$ & $-1.54(<0.01, \mathrm{~ns})$ & $-1.49(<0.05, \mathrm{~ns})$ & $1.24(\mathrm{~ns})$ \\
\hline CCL6 & $1.61(<0.0001,<0.05) \checkmark \checkmark$ & $-1.27(0.02, \mathrm{~ns})$ & $-1.56(0.05, \mathrm{~ns})$ & $1.11(\mathrm{~ns})$ \\
\hline CCL19 & $2.35(<0.001,0.01) \checkmark \checkmark$ & $-1.87(<0.01, \mathrm{~ns}) \checkmark$ & $-1.34(\mathrm{~ns})$ & $-2.77(<0.01, \mathrm{~ns}) \checkmark$ \\
\hline CCL20 & $1.67(\mathrm{~ns})$ & $-1.35(\mathrm{~ns})$ & $3.07(<0.01, \mathrm{~ns}) \checkmark$ & $-1.37(\mathrm{~ns})$ \\
\hline CCL21 & $-1.02(\mathrm{~ns})$ & $-1.48(<0.01, \mathrm{~ns})$ & $-2.55(<0.0001,0.01) \checkmark \checkmark$ & $-1.26(\mathrm{~ns})$ \\
\hline CXCL9 & $1.55(<0.01,0.02) \checkmark \checkmark$ & $-1.67(<0.01,0.03) \checkmark \checkmark$ & $-1.10(\mathrm{~ns})$ & $-1.09(\mathrm{~ns})$ \\
\hline CXCL13 & $2.24(<0.0001,<0.0001) \checkmark \checkmark$ & $-1.52(<0.01, \mathrm{~ns})$ & $-1.07(\mathrm{~ns})$ & 1.29 (ns) \\
\hline CXCL16 & $1.60(<0.0001,<0.001) \checkmark \checkmark$ & $-1.27(0.03, \mathrm{~ns})$ & $1.22(\mathrm{~ns})$ & $-1.14(\mathrm{~ns})$ \\
\hline CCR1 & $1.90(<0.01, \mathrm{~ns}) \checkmark$ & $1.00(\mathrm{~ns})$ & $-2.01(<0.01, \mathrm{~ns}) \checkmark$ & $1.13(\mathrm{~ns})$ \\
\hline CCR5 & $1.30(\mathrm{~ns})$ & $-1.17(\mathrm{~ns})$ & $-1.34(\mathrm{~ns})$ & $1.18(\mathrm{~ns})$ \\
\hline
\end{tabular}

Consistent with the effects of aging shown in Table 2, NFkB signaling, a crucial pathway regulating many inflammatory genes, is upregulated in female aging and inhibited by RLX in both female and male rats. In female rats, IFN $\gamma$, IL-6, lipopolysaccharide (LPS) and transforming growth factor (TGF) $\beta 1$ are activated in aging and inhibited by RLX. Interestingly, SMAD7, an inhibitor of TGF $\beta 1$ and fibrosis, is inhibited with aging and activated by RLX; consistent with the anti-fibrosis properties of RLX. In aged male rats, upstream analysis failed to detect an activation of the genes that were activated in aged female ventricles, yet RLX still inhibited many of them in aged male ventricles. Note that RLX inhibited several upstream regulators: hydrogen peroxide, NFkB, TLR4, IFNG, IL-6 and LPS in males and females (Table 3). 
Besides classic inflammatory gene expression that change in HF, and aging \pm RLX, RLX altered chemo-attractant genes involved in recruiting inflammatory and immune cells to sites of injury (Table 4).

\section{Signaling pathway analysis}

To identify signaling pathways that are affected by aging and RLX treatment, differentially expressed genes (DEGs) were filtered as transcripts whose absolute expression was altered by at least 1.5-fold, had expression values with RPKM (reads per kilobase of transcript per million) 1, and had a false discovery rate (FDR) $<0.05$ (Fig 3).

\section{Female rats}

Application of Ingenuity Pathway Analysis (IPA) software to DEGs showed that female rats exhibit significant activation of inflammatory and immune signaling pathways with aging (Fig. 4A) which are reversed by RLX treatment (Fig 4B). These pathways include: 1) Calciuminduced T lymphocyte apoptosis pathway, which acts to counter excessive inflammatory response [158, 159], though may be harmful in some cases [160], 2) PKC $\theta$ signaling in $\mathrm{T}$ Lymphocytes, involved in T-cell activation and NF- $\mathrm{BB}$ signaling $[161,162], 3)$ iCOS-iCOSL signaling in T-helper cells, resulting in activation and migration of lymphocytes and secretion of both antiand pro-inflammatory cytokines [163], 4) NFAT regulation of the immune response, involved in cytokine production in leukocytes [164], 5) TH1 signaling, resulting in proinflammatory response, compared to the anti-inflammatory $\mathrm{TH} 2$ signaling [165] and 6) dendritic cell maturation, which acts as a link between innate and adaptive immunity [164]. 

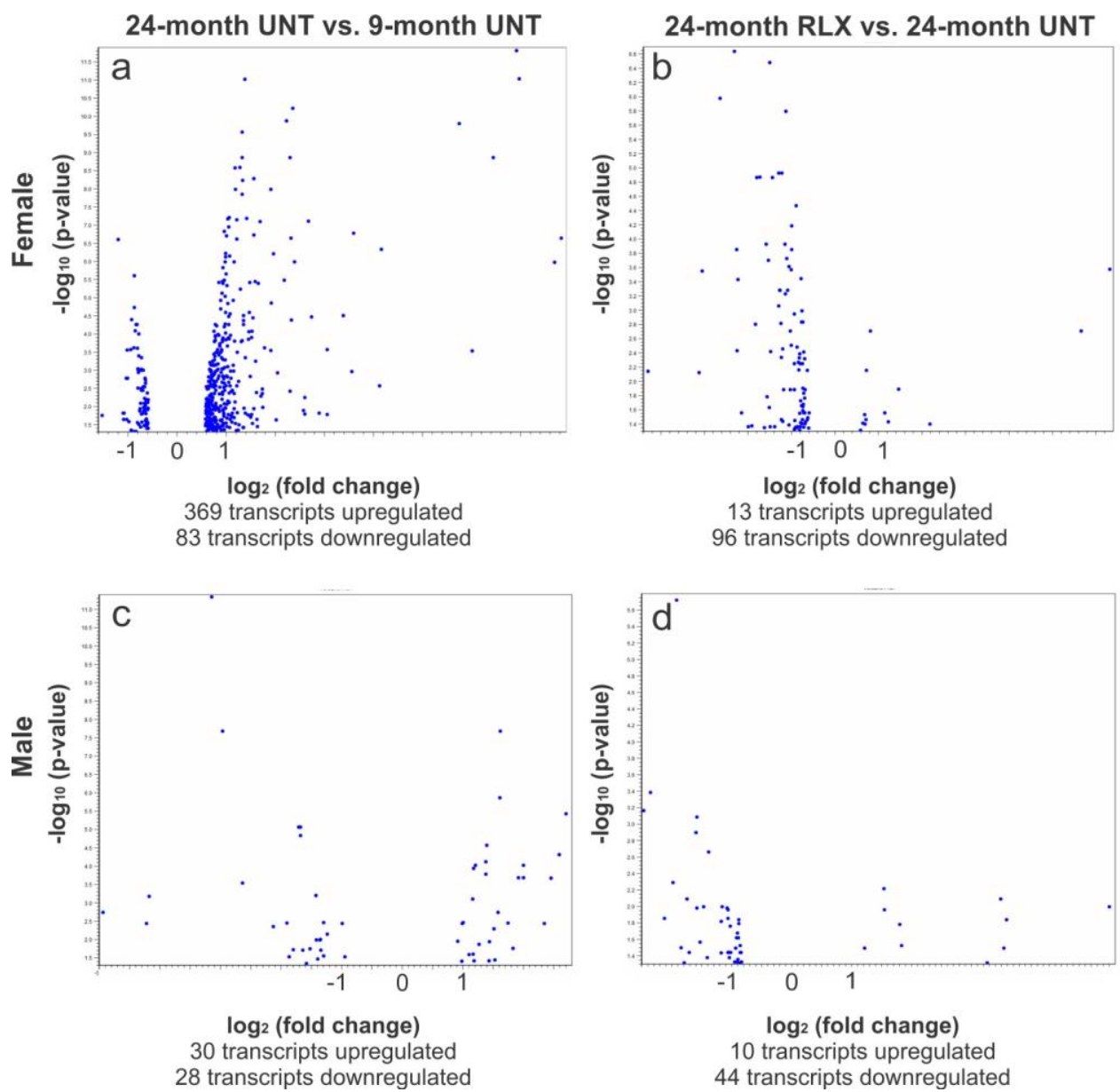

Fig 3. Volcano Plots of Differentially Expressed Genes in Aging \pm RLX treatment. A. Female aging resulted in an overall upregulation of transcripts, with fewer transcripts being downregulated. B. RLX treatment in females caused a marked suppression of most transcripts with few being upregulated. C. Transcript expression changes in males were muted compared to females during aging, with an even number of transcripts being up and downregulated. D. RLX treatment in males reflects that seen in females, with a substantial proportion of transcript alterations being suppressed compared to upregulated. Blue dots represent individual transcripts. 
Aging increased and RLX decreased expression of a common set of genes, namely those associated with the major histocompatibility complex (MHC) which is involved in immune system signaling (Table 5). This was supported by MetaCore (Thomson Reuters) analysis that showed activation of antigen presentation by MHC class I \& II in aging and inhibition with RLX treatment (data not shown). Analysis of DEGs by IPA to predict which cellular functions would be altered in aging or by RLX, showed that aged female rats exhibit increased gene expression involved in migration

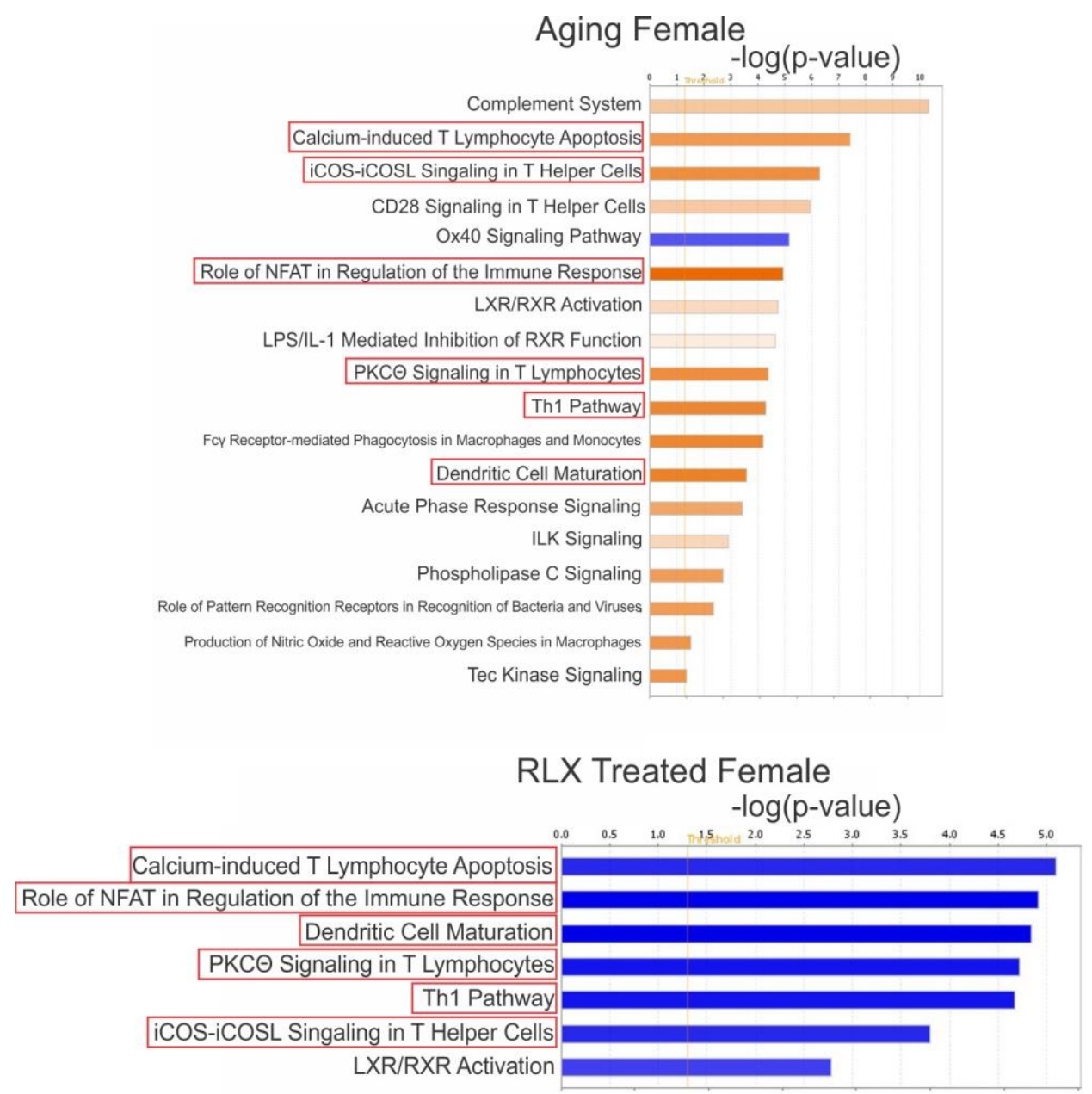

Fig 4. Pathway Analysis: Female. (a) Pathway analysis of female rats suggest that multiple inflammatory signaling pathways are activated (orange) in aging and (b) RLX treatment inhibited (blue) many of these signaling pathways. Due to the substantial number of pathways altered in female aging (93 pathways) and RLX treatment (58 pathways), only those pathways whose activation or inhibition was predicted by IPA (orange or blue colors, respectively) are shown. 
Table 5. Common genes altered by relaxin: Female. Right column indicates which genes are common between aging and RLX groups which RLX reverses compared to the aging group. Arrows indicated direction of change by RLX. FCGR3A/B: Encodes receptor for Fc portion of immunoglobin G. HLA: human leukocyte antigen; gene complex which forms the major histocompatibility complex. LOC: indicates genes with uncertain functions.

\begin{tabular}{|c|c|c|c|}
\hline Signaling pathway & Aging & RLX & RLXs Effect \\
\hline $\begin{array}{l}\text { Role of NFAT in Regulation of the } \\
\text { Immune } \\
\text { Response }\end{array}$ & Activates & Inhibits & \multirow[t]{2}{*}{$\begin{array}{l}\text { FCGR3A/FCGR3B(\#), HLA-DQA1(\#), HLA-DRA(\#), HLA-DRB5(\#), } \\
\text { LOC1009009593/RT } \\
(\#)\end{array}$} \\
\hline Dendritic Cell Maturation & Activates & Inhibits & \\
\hline $\begin{array}{l}\text { Calcium-induced T Lymphocyte } \\
\text { Apoptosis }\end{array}$ & Activates & Inhibits & \multirow[t]{4}{*}{ HLA-DQA1(\#), HLA-DRA(\#), HLA-DRB5(\#), LOC1009009593/RT(\#) } \\
\hline iCOS-iCOSL Signaling in T Helper Cells & Activates & Inhibits & \\
\hline TH1 Pathway & Activates & Inhibits & \\
\hline PKC $\theta$ Signaling in T Lymphocytes & Activates & Inhibits & \\
\hline
\end{tabular}



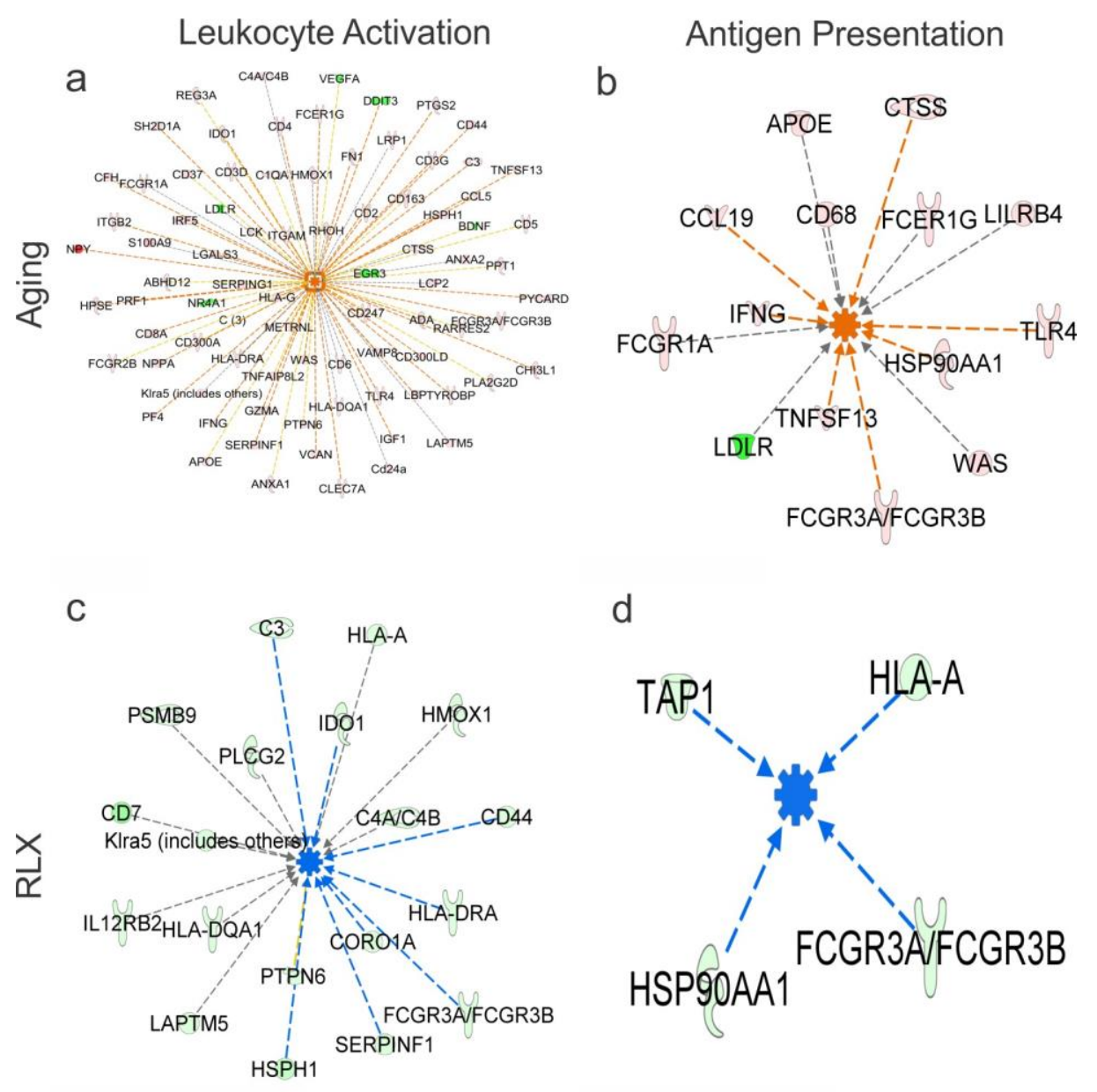

d

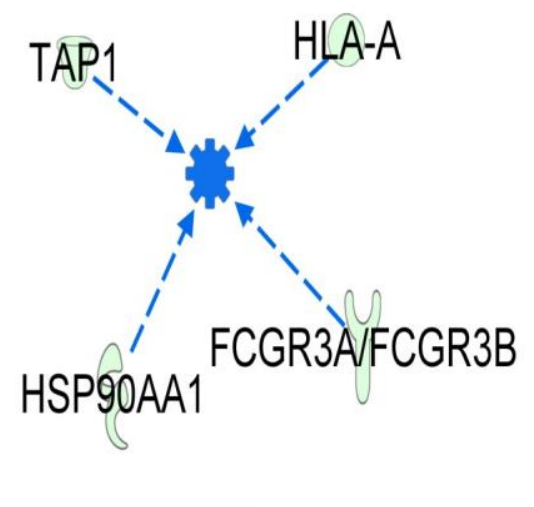

Fig 5. Cellular Function Prediction from Differentially Expressed Female Genes. IPA shows that aging leads to extensive alterations of gene expression resulting in leukocyte activation (panel a) and antigen presentation (panel b). RLX significantly reduced the expression of multiple genes associated with immune activation (panel c) and antigen presentation (panel d); that is, RLX largely reversed the effect of aging on the immune cell response. Orange centers denote activated function. Blue centers denote inhibited function. Green genes indicate reduced expression. Red genes indicated increases in expression. Orange, Blue and Yellow lines: the altered genes lead to activation, inhibition of function or is acting in a way opposite to the current literature, respectively. Brown lines: the function of the gene is unclear in the literature. 
and activation of leukocytes and increased antigen presentation (Fig 5A and 5B), and these functions are significantly reversed by RLX (Fig 5C and 5D), supporting RLXs role in MHC regulation.

In addition to the signaling pathways that are reversed by RLX based on IPA, MetaCore analysis also showed that the classical complement cascade is significantly activated in aging (Fig 6A) and reversed by RLX treatment (Fig 6B) in female rats.

\section{Male rats}

IPA pathway analysis of male rats ( $\underline{\text { Fig } 7 \mathrm{~A} \text { and } 7 \mathrm{~B})}$ show a significant decrease in dendritic cell maturation with age (Fig 7A) and inhibition of EIF2 and IL-6 signaling with RLX treatment $(\underline{\text { Fig } 7 \mathrm{~B}})$. These data, again, support the notion that there are major differences in the regulation of gene expression in male and female rats with aging and RLX treatment. MetaCore analysis in male rats suggest that multiple pathways are inhibited by RLX: IL-1, CCR1, CCL2, HMGB1/RAGE and LPS signaling, though these are not necessarily activated by aging (not shown). IPA analysis in male rats, did not show an activation of immune cell recruitment in aging, though RLX significantly repressed immune cell activity, recruitment and accumulation (Fig 7C).

These data and Tables $\underline{2}-\underline{4}$ indicate that gender plays a vital role in terms of which genes are altered in aging and disease, and that treatments can affect males and females in different ways. 

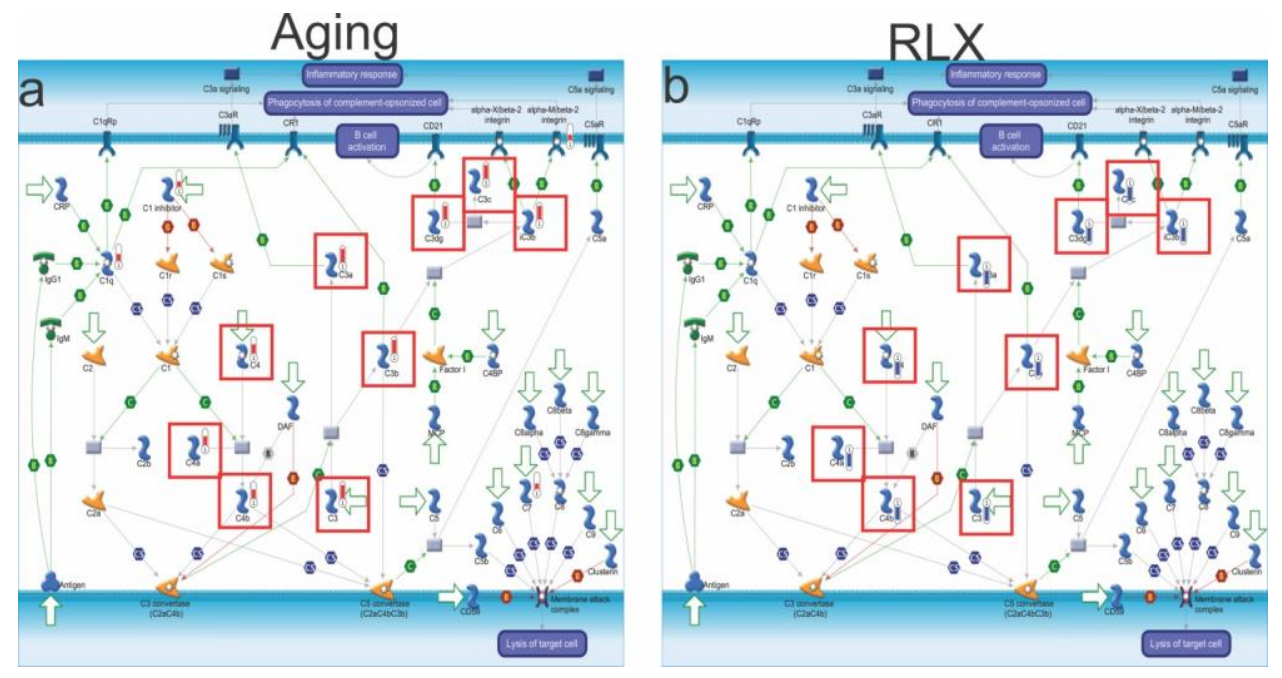

Fig 6. Complement Cascade Activity with Aging and Relaxin: Female. The classical complement cascade can result in an opsonization, or marking of cells for phagocytosis, and lysis of bacteria. It can also result in an augmented inflammatory response. A. Effect of aging on complement component activation. B. Effect of RLX on reversal of complement activity. Red boxes indicate significant alterations of specific complement components. Red and blue thermometer markers next to specific components indicate increased and decreased component expression, respectively. For key describing complement components, see https://portal.genego.com/help/MC legend.pdf.

\section{Macrophage accumulation}

Based on higher IFN $\gamma$, IL-6, MHC gene expression, increased complement activation, and prediction of increased inflammatory/immunce cell activity by IPA in aging female but not male rats, we first investigated the effects of aging on macrophage and T-cell recruitment in female ventricular tissue by immunofluorescence (Fig 8A-8C). Tissue macrophage infiltration measured with the marker F4/80 [166], increased significantly in aging (ii 8B) compared to the young controls (Fig 8A) and was dramatically decreased with RLX treatment (Fig 8C). Next, we tested the male LV for macrophage accumulation (Fig 8D-8F). Male rats had significantly less macrophages that did the corresponding female rats in each group. Moreover, male aging (Fig 8E) did not significantly increase macrophage accumulation compared to the young male rats (Fig 8D), and RLX did not cause a significant change compared to young or aged male controls 
(Fig 8F). Although RLX slightly increased and decreased T-cell accumulation in females and males, respectively, the significance is unclear because T-cells were sparse $(<6 /$ high power field) in all groups (data not shown).

Our data show that aging promotes an inflammatory response in females, but not males, and RLX reverses these effects in females, and suppresses many inflammatory genes in males.

One may reasonably conclude that RLX is acting at a genomic level to suppress inflammatory and immune responses in male and female rat heats. Others studies have bemoaned that only $10 \%$ of immunology studies consider sex as an important variable [167]; and the current findings re-enforce this concern by showing that females and males exhibit drastically different gene expression patterns and signaling pathway regulation, not only in aging, but also by relaxin therapy.

Reductions in IL-6 have been associated with beneficial effects in rheumatoid arthritis, though with increased LDL in the plasma.[138] Here, we showed that aging increased IL-6 transcription in females, while also significantly lowering the LDL receptor necessary to reduce LDL concentration in the plasma. However, RLX reduced IL-6 and increased LDLR, suggesting more LDL receptors and a greater efficacy at removing excess LDL cholesterol from the plasma. These results suggest RLX may prove to be a beneficial therapy for patients suffering from rheumatoid arthritis or coronary artery disease. In addition, IL-6 has been associated with type II diabetes development, and as such, RLX may prove to be a beneficial therapy in diabetes management [타. 


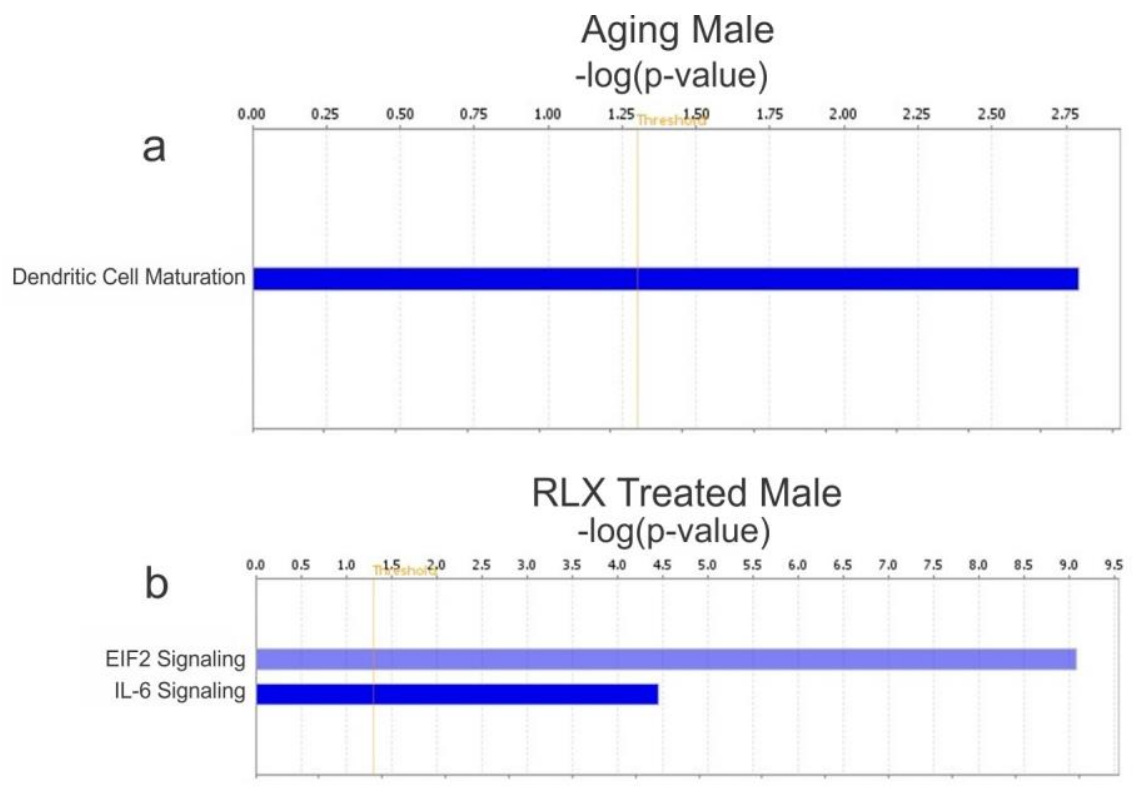

\section{Function Analysis - Male}

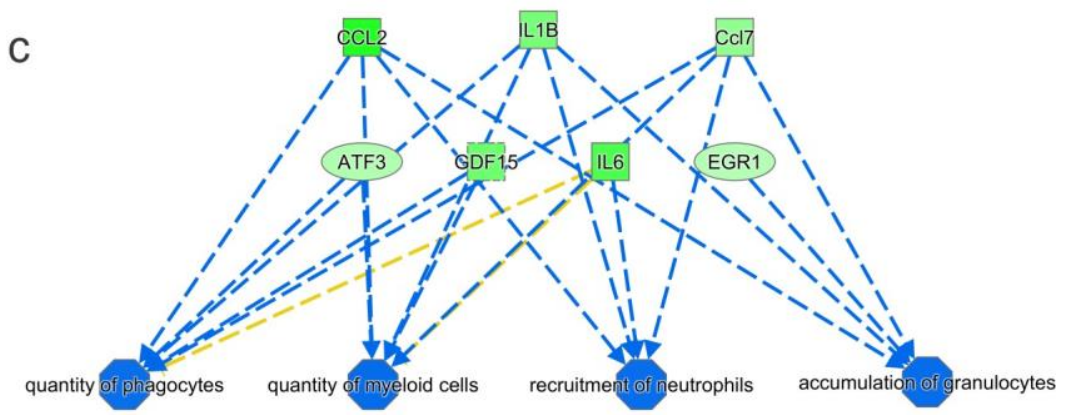

Fig 7. Pathway Analysis: Male. Male rats had negligible pathway alterations (a) with aging or (b) RLX treatment. Blue bars indicate pathway inhibition. IPA in males (c) showed that quantity, recruitment and accumulation of various immune cells were inhibited (green) by RLX. In males, aging and RLX treatment altered 24 and 46 pathways, respectively; hence only pathways whose activation or inhibition was predicted by IPA (orange or blue colors, respectively) are shown here. 
The classical complement cascades converge on C3 components, which are necessary for C5 convertase formation and lead to inflammation (the anaphylatoxins C3a, C4a and C5a components) or bacteria lysis (C5b) [134]. Our data (Fig 6) shows that the C3a and C4a complement components are indeed two pieces, upregulated by aging and reversed by RLX. The anaphylatoxins can also increase activation of macrophages, resulting in increased release of inflammatory cytokines and stimulate the release of proteolytic enzymes which contribute to ongoing tissue damage and increase endothelial surface thrombogenicity. Moreover, clinical trials have shown that inhibition of C3 convertase attenuates both leukocyte attraction and mortality in cardiopulmonary bypass patients [134]. Taken together, our data suggest that relaxin could be a potent therapy to regulate aberrant complement activity.
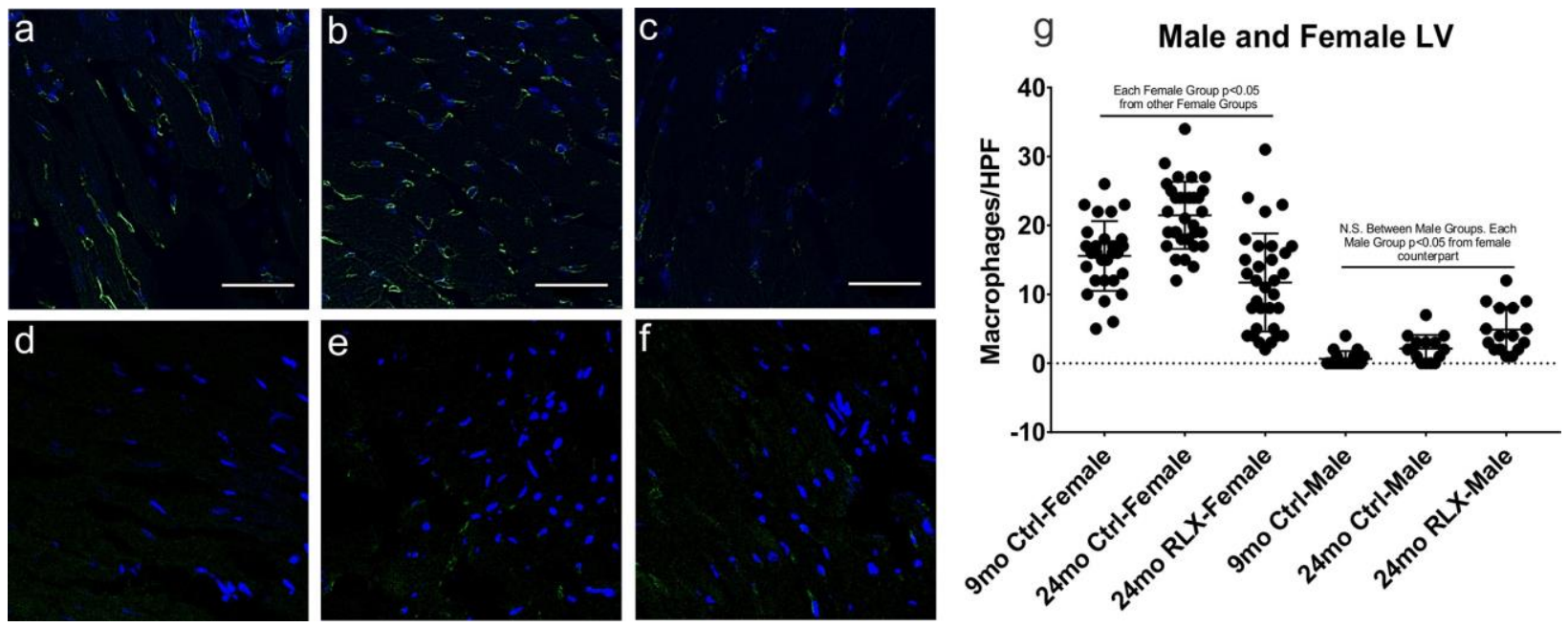

Fig 8. Effect of Aging and Relaxin on Macrophage Accumulation in the Heart. A-C. Female. Aged female left ventricles had significant macrophage accumulation (B. 21.47 \pm 0.86 macrophages/hpf) compared to young controls (A. 15.57 \pm 0.96 ) and RLX reversed this aged-dependent macrophage accumulation (C. 11.72 \pm 1.26 ). D-F. Male. Male left ventricles showed no change in macrophage accumulation in aging (E.

$2.13 \pm 0.51)$ or with RLX treatment (4.88 \pm 0.84$)$ compared to young $(0.65 \pm 0.27)$ male rats, and had less macrophages than females in each group. G. Quantification of macrophage accumulation. Blue: DAPI; Green: F4/80 macrophage marker; HPF: high power field. Data is reported as mean \pm SEM. N.S.: Not significant. 60x magnification. Scale bar: $50 \mu \mathrm{m}$.

Hofmann and Frantz [136] suggested that targeting specific cytokines in pre-clinical studies and clinical trials have failed to produce beneficial results due to the redundant nature of 
the immune system. Our study shows that RLX significantly alters multiple cytokines and signaling pathways in both genders, which could lead to improved suppression of inflammatory related pathophysiology that rely on these redundant immune effects.

Franceschi and Campisi [154] report that there are at least eight possible mechanisms for “inflammaging”: 1) persistent leukocyte production of reactive molecules, 2) cytokine production from damaged non-immune cells, 3) alterations in anabolic signaling, (e.g. TNF $\alpha$ and IL6 signaling) altering insulin production, 4) "self-debris" from cell injury that accumulates over time, activating the inflammatory response, 5) reduced ability of the aged body to sequester infiltrated bacteria or viruses, 6) cellular senescence, 7) release of cytokines from damaged mitochondria and 8) dysregulation of the complement system. Our data supports reasons 1, 3 and 7 in that aging increases cytokine transcript production and RLX reduces cytokine transcript expression and accumulation of macrophages in LV tissue in females. Needless-to say, further studies are needed on all possible mechanisms. Inflammation has been implicated in many aspects of cardiovascular disease including LV remodeling [169], hypertrophy [141], stroke [140], HF [139], and AF [170]. The suppression of inflammatory responses by RLX warrants further investigation considering its possible beneficial actions towards a therapy for such inflammatory related diseases as AF, diabetes, obesity and HF.

A limitation of this study is the lack of a general consensus for quantification and analysis of RNA-seq data [171]. The cut-off for filtering by RPKM is arbitrary and varies greatly in the literature, from 0.1 [172] to greater than 5 [173]. To determine which RPKM value to use for gene filtering, we obtained a freely available RNA-seq dataset analyzed using TopHat software [174], and compared results using our current analysis software. An RPKM 1 showed good agreement among the results between the two software packages. Another limitation is that 
RNA-seq was performed only on LV tissue, and it is unknown if atria or RV behave similarly. Finally, the experiments cannot discriminate whether RLX is removing the inflammatory stimulus resulting in downregulation of inflammatory markers, or is regulating transcription of the inflammatory markers themselves. The long-term effects following a short-term administration of RLX in clinical trials suggests a direct effect on transcription, and the broad reduction in multiple genes suggests a common nexus of action. Evidence suggests that multiple proinflammatory cytokines are controlled at the transcriptional level through histone modifications or phosphorylation.[175] Our lab showed that RLX can regulate phosphorylation of proteins [14], hence future studies should look into RLX's ability to regulate histone modification and phosphorylation of genes. 


\subsection{Relaxin Acts Through Wnt Signaling}

As discussed above, aging is associated with structural and functional changes of the cardiovascular system and is a major risk factor for a number of pathologies, including atrial fibrillation (AF) and heart failure (HF).[8,129,176] The prevalence of age-associated cardiovascular disease $(\mathrm{CD})$ is expected to increase steadily, with estimates reaching 70 million people in the United States by 2030.[129] Structural and electrical remodeling during aging includes excess fibrosis, cellular hypertrophy and vascular stiffening leading to hypertension, reduced myocyte contractility, diastolic and/or systolic dysfunction and HF.[129,176,177] Agedependent electrical remodeling involves ion channel and gap junction dysregulation which result in increased arrhythmia susceptibility.[13,14,177,178] We previously showed that the hormone relaxin (RLX) suppressed AF in aged (24-months old) rats by increasing conduction velocity (CV) of atrial action potentials.[13] These effects were linked to increased expression of the voltage-gated sodium channel Nav1.5, increased current (INa), and a marked decrease in fibrosis.[13] However, the mechanisms whereby RLX acts to increase Nav1.5 and reduce fibrosis are largely unknown, though studies indicate that RLX suppresses TGF $\beta$ and matrix metalloproteases in fibrosis regulation. $[13,14,43]$ We reported that RLX-treatment of young (9months) and aged (24-months) rat (F-344 strain) ventricles resulted in a reversal of inflammatory and immune responses and an overall rejuvenation of cardiac properties.[8]

Canonical Wnt signaling is a master controller in cardiac embryogenesis and development, but it is thought to be quiescent after birth. [82,178] In the absence of Wnt 
signaling, $\beta$-catenin is phosphorylated, marked for ubiquitination and removed by the proteasome. During canonical Wnt-signaling, Wnt binding to Frizzled and the co-receptors, LRP5/6, results in the dissociation of the $\beta$-catenin destruction complex causing the accumulation of $\beta$-catenin, which enters the nucleus to regulate the activity of the TCF/LEF family of transcription factors. Exogenous Wnt3a is routinely added to stimulate the differentiation of human induced pluripotent or embryonic stem cells into cardiac myocytes and conversely, blockade of Wnt-signaling with Dickkopf-1 (Dkk1) inhibits cardiac differentiation.[179] Interestingly, late antagonism of endogenous Wnts enhances cardiogenesis, indicating a biphasic role in human cardiac differentiation.[180,181] Besides the regulation of the contractile apparatus, Wnt-signaling regulates the expression of the voltage-gated $\mathrm{Na}+$ channel Nav1.5 in neonate rat ventricular cardiomyocytes (NRVM) [82] and HL-1 cells.[182] Puzzlingly, Wnt3a suppressed Nav1.5 expression in NRVM,[82] whereas activation of canonical Wnt signaling by other means increased Nav1.5 and Connexin-43 (Cx43), and reduced ventricular ectopic beats in NRVM, 3-week and 6-month old mouse models of arrhythmogenic cardiomyopathy.[81,84,183]

Here, we tested the hypothesis that the effects of RLX on Nav1.5 and fibrosis are mediated by canonical Wnt signaling. While RLX and Wnt signaling have been investigated in models of cancer [184-186], there is no work on the interactions of the RLX and Wnt pathways in adult heart and 'healthy' aging as a precursor of cardiac diseases. We focused on hearts from 9-month (adult) and 24-month-old (aged) Fischer 344/Brown Norway F1 (F-344) male rats obtained from the National Institute of Aging. F-344 rats have been extensively studied and characterized as a model of aging because of their linear aging, and because, like humans, they exhibit an age-dependent excess of cardiac fibrosis and susceptibility to atrial 
fibrillation.[13,132] Rats were treated with RLX $(400 \mu \mathrm{g} / \mathrm{kg} / \mathrm{day})$ or vehicle (sodium acetate) via subcutaneous osmotic mini-pumps for 14 days, resulting in an increase in the circulating plasma concentration of RLX from $0.017 \pm 0.027 \mathrm{ng} / \mathrm{mL}$ to $23.76 \pm 10.60 \mathrm{ng} / \mathrm{mL}$, as determined by an ELISA assay.

\section{Relaxin improves ventricular function}

Hearts were perfused in a Langendorff apparatus for optical mapping of atrial and ventricular action potentials to calculate conduction velocity (CV).[13,14] Aged ventricles treated with RLX showed a marked increase of CV, an effect that was particularly pronounced at fast pacing rates compared to aged control ventricles (Fig. 9Aa). CV restitution kinetics measured by programmed stimulation revealed that premature stimuli elicited depolarizing waves in control aged rats with considerably slower CV than in RLX treated aged-rats (Fig. $9 \mathrm{Ab})$. It is interesting to note that when paced at cycle lengths below $150 \mathrm{~ms}$, control aged ventricles failed to capture and propagate or maintain a steady state CV whereas RLX-treated ventricles could be paced at those faster rates without significant decrement of CV (Fig. 9Aa). In ventricles of RLX treated rats, the slope of the restitution kinetics curve becomes less steep than for untreated animals which suggests a decrease in arrhythmia vulnerability (Fig. 9Ab).

A hallmark of advancing age is an escalation of excess fibrosis. Increased collagen deposits are associated with cardiac dysfunction due to disruption of cell-cell coupling and a depolarization of the resting membrane potential.[187] Collagen content was measured by staining left ventricular (LV) sections with Picro-Sirius Red and by calculating the collagen to tissue ratio in RLX treated and control animals. RLX treatment significantly reduced the collagen to tissue ratio (Fig. 9Ba-c). Most important, RLX reversed age-associated fibrosis to levels like those measured in 11-month-old rats.[152] 
In addition, reductions in Nav1.5 expression contribute to arrhythmia and other heart pathologies by slowing CV.[22] RLX significantly increased Nav1.5 expression in LV sections compared to control (Fig. 9Ca-c).

Further, $\beta$-catenin was reduced in LV sections of aged (Fig. 10d) compared to adult (Fig. 10a) rat hearts and was diffusely distributed in the cytosol with relatively little localization at intercalated discs (IDs) while Cx43 was localized to the lateral membranes in aged vs adult LV (Fig. 10e vs. 10b). RLX shifted the localization of Cx43 from the lateral membranes to IDs, increased the levels of $\beta$-catenin, and $\beta$-catenin became co-localized with $\mathrm{Cx} 43$ at IDs (Fig. 10gf). In general, LV of aged-rats with RLX-treatment resembled the LV of adult rats (Fig. 10a-c). This strongly suggested that RLX treatment reversed some of the aging-induced changes observed in 24-month-old rats. Similar findings were seen in aged atria (Fig. 11A).

\section{Relaxin acts via Wnt-signaling in aged rats}

Based on previous work of Wnt signaling effects on Nav1.5 and ID proteins, we tested the role of Wnt-signaling in RLX-mediated upregulation of Nav1.5 in adult and aged animals. RNA from aged atria \pm RLX-treatment followed by RT-PCR showed the expected reduction of fibrosis mRNA biomarkers (Metalloproteinases (MMPs: MMP-2 and 9, $\alpha$-smooth muscle actin) but also showed a marked increase in Wnt1 expression (Fig.11-Table). Likewise, RLX-treatment increased Wnt1 expression in LV sections (Fig. 11B). Next, tissue from aged LV \pm RLX was tested for Dickkopf-1 (Dkk1) expression, an intrinsic inhibitor of Wnt signaling. Dkk1 has been shown to bind LRP6 and blocks canonical signaling of all Wnt ligands.[188] RLX-treated LV showed a significant reduction of Dkk1 compared to control LV (Fig. 12). These data suggest that RLX acts to activate Wnt signaling by two complementary mechanisms: increasing Wnt1 expression and reducing Dkk1 and Wnt inhibition. 

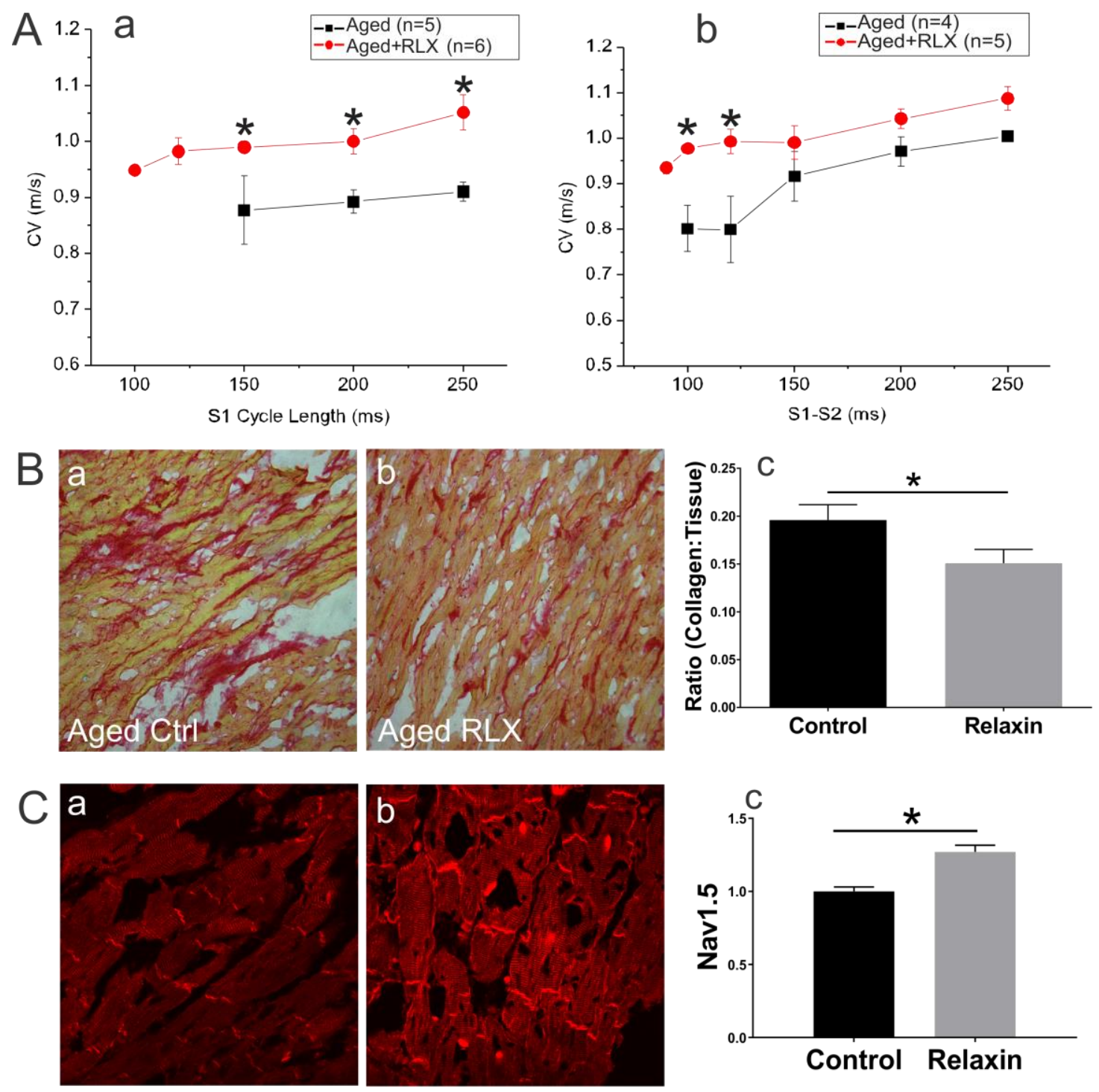

Figure 9: Relaxin Effects on Conduction Velocity and Collagen Expression in Aged Hearts. A: a. RLX treatment resulted in a significant increase in CV during steady pacing (S1:S1). b. CV restitution kinetics measured by programmed stimulation with premature pulses (S2 beats) was significantly increased by RLX, particularly during fast pacing times. * indicates $\mathrm{P}<0.05$. 20x magnification $\mathrm{B}$ : LV tissue sections were stained with Picro-sirius red and imaged on an Olympus Provis Light Microscope. The collagen to tissue ratio was significantly reduced in RLX $(\mathrm{Bb}, \mathrm{n}=6)$ treated animals compared to control $(\mathrm{Ba}, \mathrm{n}=5)$ by $23 \%$. C. LV tissue stained for Nav1.5 showed that $\mathrm{RLX}(\mathrm{Cb}, \mathrm{n}=6)$ significantly increased Nav1.5 expression compared to control hearts $(\mathrm{Ca}, \mathrm{n}=5)$. 60x magnification. 

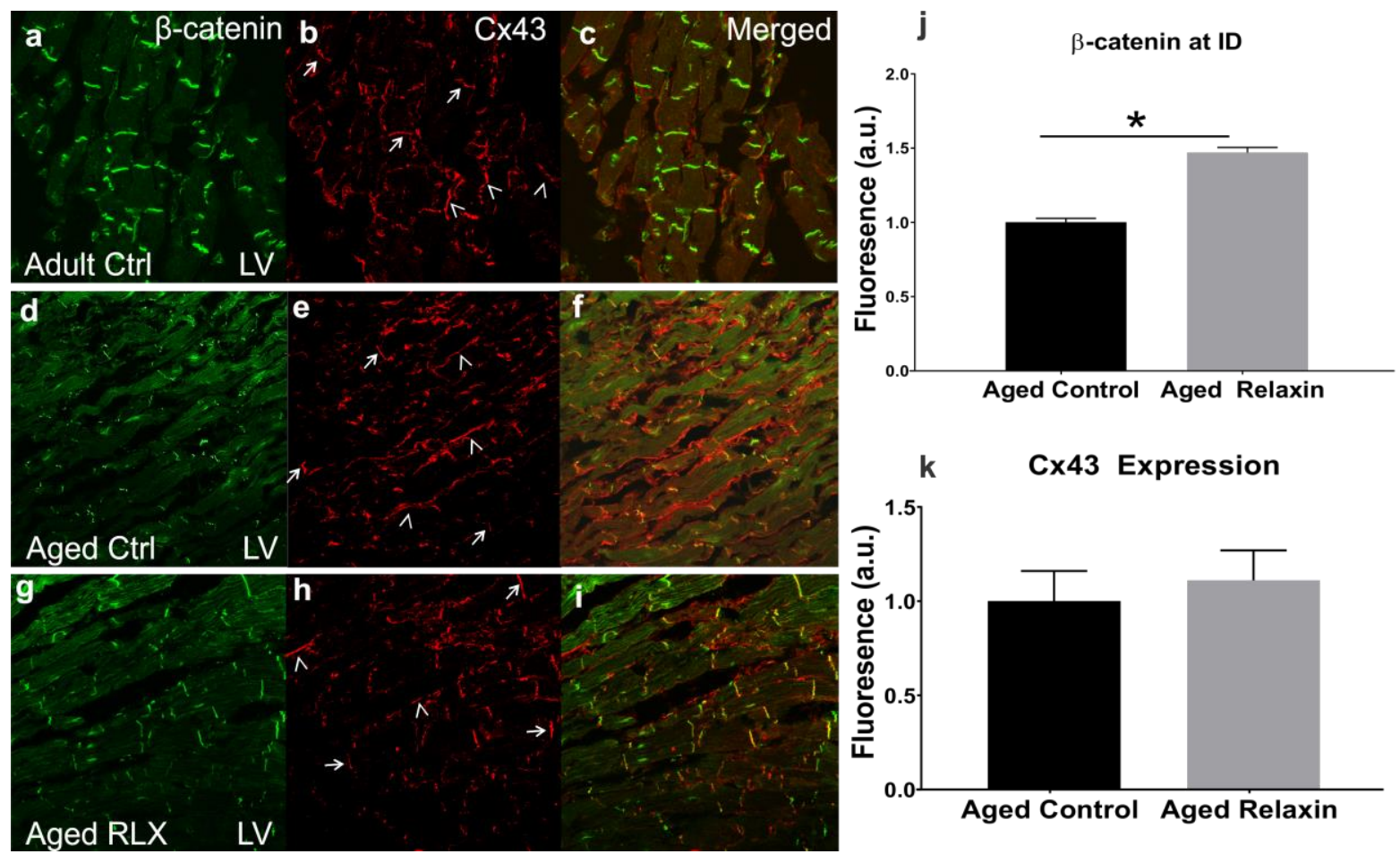

Figure 10: Relaxin Effects on Intercalated Disk Proteins in Left Ventricle. In adult (9-months, $\mathrm{n}=4)$ F-344 rats (Aa-c), $\beta$-catenin and Cx43 localized to the intercalated disks (arrows), with minimal movement of $\mathrm{Cx} 43$ to the lateral membranes (arrow heads). Untreated aged (24-months, $\mathrm{n}=4$ ) rats (Ad-f) exhibited a marked reduction in $\beta$-catenin and translocation of $\mathrm{Cx} 43$ to the lateral membranes. RLX treatment ( $\mathrm{n}=4)$ of aged animals (Ag-f) showed that RLX can reverse the effects of aging on $\beta$-catenin expression and results in trafficking of Cx43 to the intercalated disk, matching closely that seen in 9-month-old rats. Aj-k. Quantification of $\beta$-catenin/Cx43 expression. 60x magnification. 

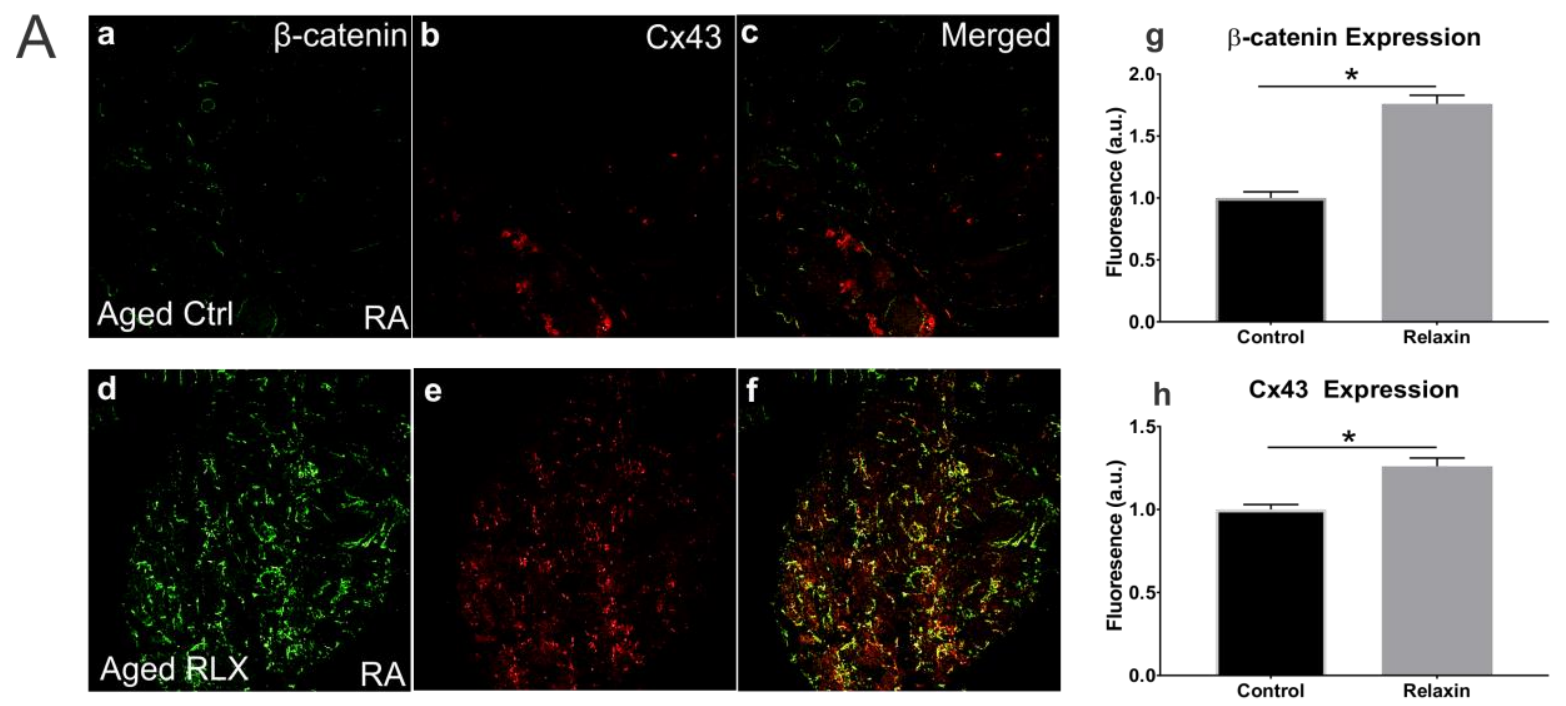

Table. Fibrosis and Wnt-related gene expression for RLX treated aged atria. " Relative to aged control.

\begin{tabular}{c|cc}
\hline Protein & Gene & Expression* $^{*}$ \\
\hline MMP-2 & MMP2 & $\downarrow 43 \%$ \\
MMP-9 & MMP9 & $\downarrow 41 \%$ \\
$\alpha S M A$ & ACTA2 & $\downarrow 30 \%$ \\
Wnt1 & WNT1 & $\uparrow 80 \%$ \\
\hline
\end{tabular}
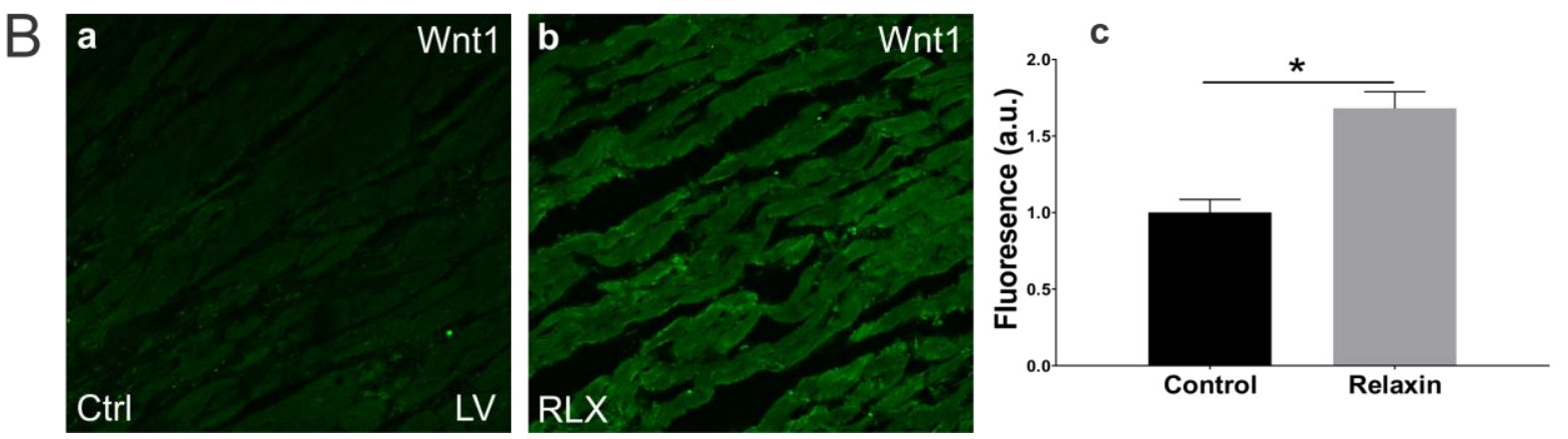

Figure 11: Relaxin Effects on Intercalated Disk Proteins in Atria. Aa-c. Aged right atrial tissue (Aa-c) expressed significantly less $\beta$-catenin and Cx43 than RLX treated animals (Ad-f). Ag-h. Quantification of atrial $\beta$-catenin and Cx43 expression (n=5/group). 60x magnification. Table 1: Relaxin altered expression of mRNA relating to fibrotic and Wnt related genes. B. RLX (Bb) increased Wnt1 expression in LV compared to control (Ba). Bc. Quantification of Wnt1 expression in LV sections. 60x. 


\section{RLX-Wnt interaction in isolated cardiomyocytes}

The effects of RLX were studied in isolated adult left ventricular myocytes by culturing the cells in 96 well plates with various doses (0-100 nM) of RLX for 2-days (Fig. 13a-b). After treatment, the myocytes were fixed, labeled with Nav1.5 antibody and fluorescein-conjugated secondary antibody and fluorescent phalloidin-labeled actin used as a counterstain for normalization purposes. Fluorescence intensities showed that RLX up-regulated Nav1.5 with an EC50 of $1.3 \mathrm{nM}$ (Fig 13c-e), which is consistent with the reported affinity of RLX for its cognate receptor, RXFP1.24
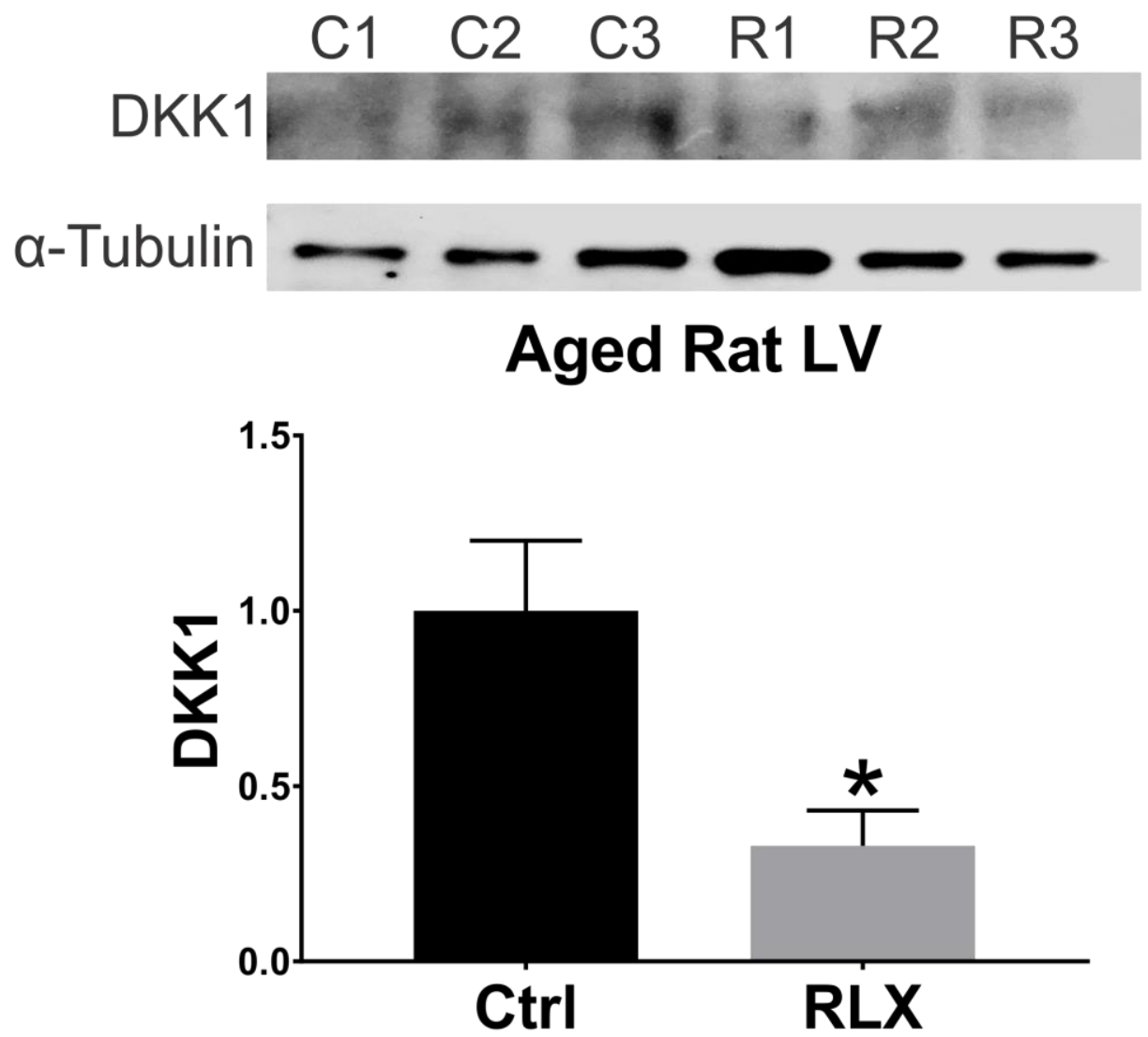

Figure 12: Relaxin Effects on Dikkpof-1 Expression: Whole Left Ventricle. Relaxin significantly reduced DKK1 expression compared to control hearts. $\mathrm{C}=$ control. $\mathrm{R}=$ Relaxin. $\mathrm{n}=3$ /group. 


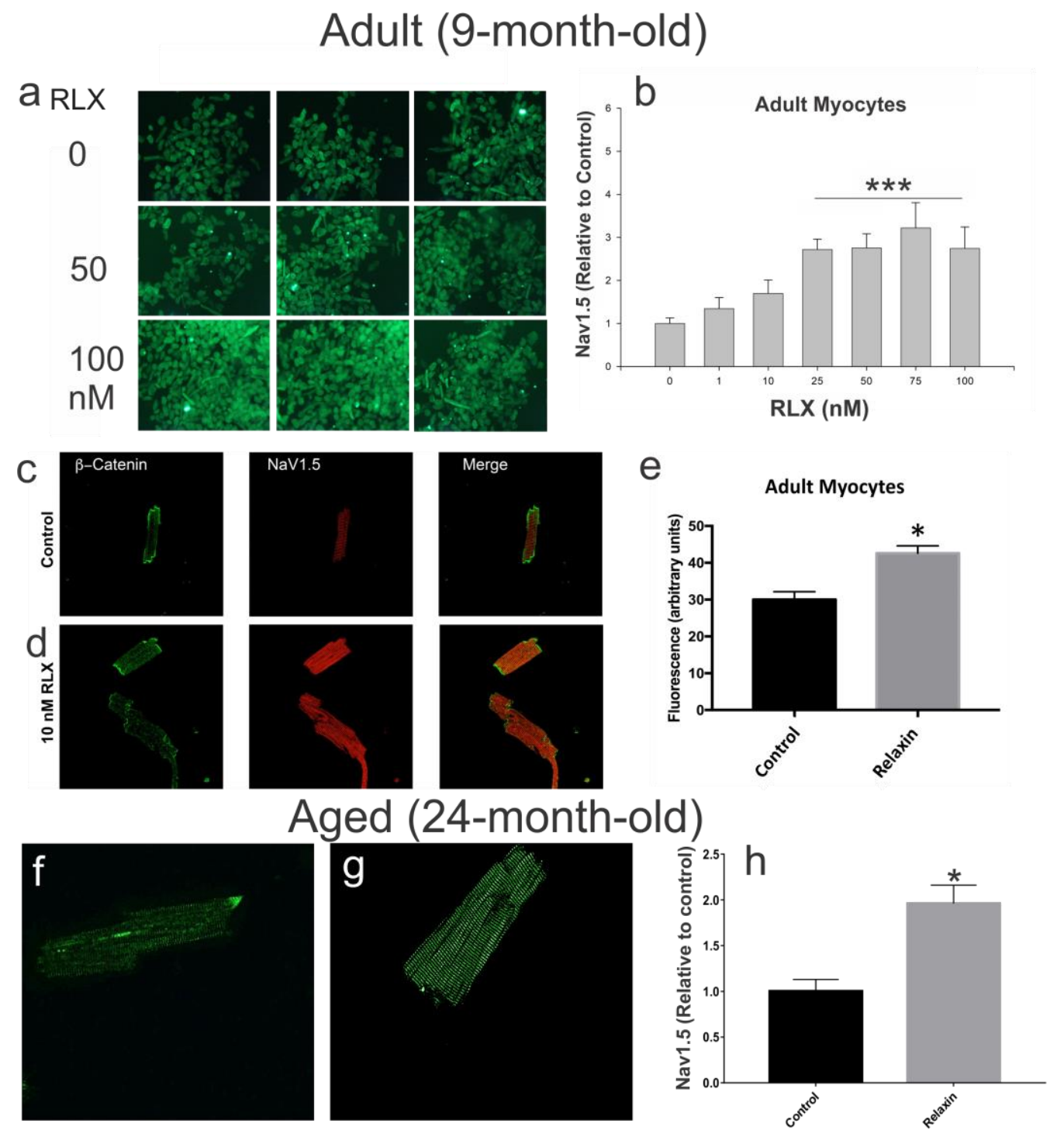

Figure 13: Relaxin Effects on Nav1.5 in Left Ventricular Cardiomyocytes. A-B: Rat ventricular myocytes treated with RLX in a graded manner followed by fixation and labeling with Nav1.5 Ab show that Nav1.5 fluorescence visibly increases with RLX treatment. Histogram: The ratio of Nav1.5 to phalloidin vs. [RLX] showed that Nav1.5 increased 3-fold at $25 \mathrm{nM} \mathrm{RLX;} \mathrm{EC50} \mathrm{1.3} \mathrm{nM,} \mathrm{(n=6} \mathrm{wells/bar,} \mathrm{P<0.01).} \mathrm{C-D.} \mathrm{RLX}$ significantly increased Nav1.5 expression in isolated myocytes compared to control by $30 \%$ ( $\mathrm{n}=10-12$ cells/group). 60x magnification. 
As shown in Figure 10, RLX increased $\beta$-catenin and Cx43 trafficking to the ID. To determine whether RLX also acted via a Wnt-related mechanism in isolated myocytes, we incubated freshly isolated ventricular myocytes with RLX (25 nM; 24-48 hours) or vehicle (Na+ acetate) and then double stained with DAPI (nuclear stain) and a $\beta$-catenin antibody to measure the number of myocytes that expressed nuclear $\beta$-catenin. Control myocytes had a considerably smaller fraction of $\beta$-catenin-containing nuclei (Fig. 14Aa,c) compared to RLX treated myocytes (Fig. 14Ab,c), suggesting that RLX is acting in part by activating canonical Wnt signaling in isolated cardiomyocytes.

To determine if Wnt signaling is directly involved in Nav1.5 regulation, myocytes were treated with either CHIR-99021 (a GSK3 $\beta$ inhibitor, $3 \mu \mathrm{g} / \mathrm{mL})$, RLX (25 nM) \pm Dkk1 (0.1 $\mu \mathrm{g} / \mathrm{mL})$ or Wnt1 $(0.1 \mu \mathrm{g} / \mathrm{mL}) \pm$ Dkk1 for 24 hours. Activation of Wnt signaling by the GSK3 $\beta$ inhibitor, CHIR-99021 resulted in a significant increase in Nav1.5 expression to the same extent as RLX alone and compared to control myocytes. The simultaneous treatment of myocytes with CHIR-99021 and RLX did not result in a further increase of Nav1.5 expression (Fig. 14Ba-e). Inhibition of Wnt signaling by Dkk1 significantly blocked RLX (Fig. 14f-j) and Wnt1's (Fig 14Bk-o) effects on Nav1.5 expression. These results strongly suggest that, contrary to the effects reported in NRVM, canonical Wnt signaling in adult cardiomyocytes increases Nav1.5 expression.

The effect of RLX on Dkk1 seen in rats treated with the hormone was also studied in isolated cardiomyocytes by treating the cultured myocytes with Na-acetate (control) or RLX for 2-days followed by labeling with an anti-Dkk1 antibody. In control myocytes, Dkk1 protein appeared as broadly distributed punctate spots inside the cells (Fig. 15 arrows).25 In contrast, RLX treatment significantly reduced the expression of Dkk1 in cardiomyocytes (Fig. 15). Thus, 
at least in part, RLX activates Wnt signaling in both cardiomyocytes and LV tissue through a downregulation of constitutively expressed DKK1.

\section{Interplay between RLX and Wnt in fibroblasts}

Fibrosis is a major contributor to impaired cardiovascular function and diastolic heart failure and has been shown to increase with age.[152] We have shown that RLX significantly reduces fibrosis in the atria [13] and ventricles of aged rats (Fig. 9). TGF $\beta$ significantly increased the differentiation of fibroblasts into myofibroblasts as determined by the increased levels of collagen I and F-actin, and RLX blocked these effects (Fig 16). The suppression of the differentiation of fibroblasts to myofibroblasts by RLX has been reported in neonate or immortalized cell lines $[43,187]$ but these cells do not necessarily respond to RLX in the same manner as adult primary cardiac fibroblasts and cannot be used as evidence of the anti-fibrotic actions of RLX in animals. To determine the role of Wnt signaling on the effects of RLX in cardiac fibroblasts, adult F-344 cardiac fibroblasts were isolated, cultured with RLX and/or TGF $\beta$ and were treated with recombinant Dkk1 for 72 hours, after which, collagen levels were measured by immunofluorescence. TGF $\beta$ significantly increased collagen compared to control (Fig. 17a,b) and RLX blocked TGF $\beta$ 's effects (Fig. 17b). The addition of Dkk1 resulted in a significant reversal of the effects of RLX on collagen expression (Fig 17g). Dkk1 had no effects on RLX alone (Fig. 17f). These data are summarized in the bar graph shown in Fig 17d. These findings are consistent with RLX's action in ventricular myocardium. Furthermore, RLX increased the expression of Wnt1 in cardiac fibroblasts (Fig. 18). These data strongly support our hypothesis: RLX increases activation of canonical Wnt signaling, and the effects of RLX on gene expression and TGF $\beta$ signaling are significantly inhibited by the canonical Wnt signaling inhibitor Dkk1. 

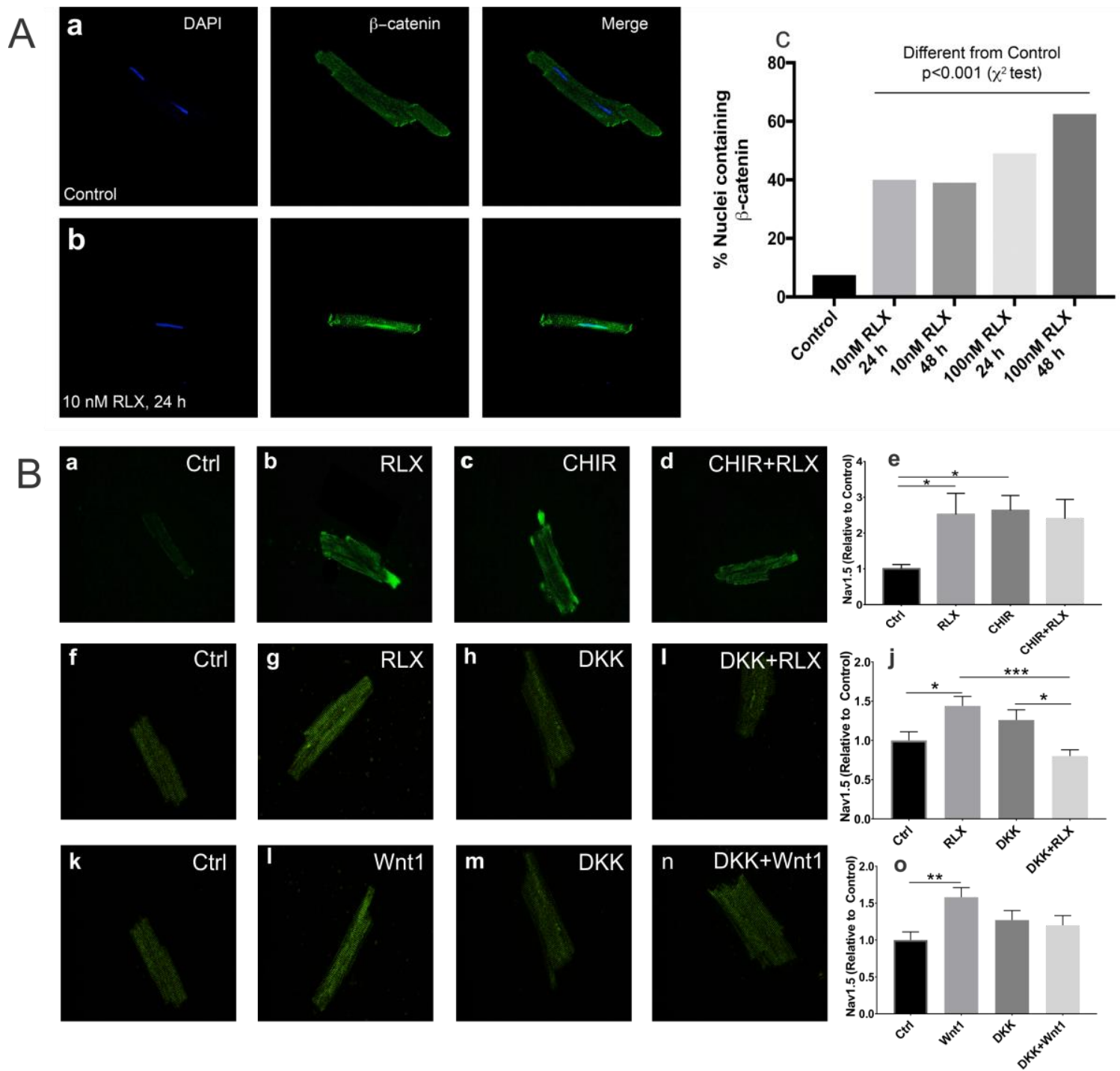

Figure 14: Relaxin Signals Through Wnt Signaling to Increase Nav1.5. A. Less than $10 \%$ of untreated myocytes exhibit nuclear $\beta$-catenin (Aa,c); however, cells treated with RLX for 24 or 48 hours showed a significant increase in cells positive for nuclear $\beta$-catenin (Ab-c). 60x magnification. B. Cells treated with RLX or CHIR, an inhibitor of GSK3 $\beta$ and Wnt pathway activator, significantly increased Nav1.5 by more than 2-fold (Ba-e, $\mathrm{n} \geq 8$ cells/group). Inhibition of canonical Wnt signaling by DKK1 blocked the effects of RLX $9 \mathrm{n} \geq 24$ cells/group) and Wnt1 $(\mathrm{n} \geq 17$ cells/group) on Nav1.5 expression (Bf-o). 60x magnification $*$ indicates $\mathrm{p}<0.05$ compared to controls. $*$ indicates $\mathrm{p}<0.05$. $* *$ indicates $\mathrm{p}<0.01$. $* * *$ indicates $\mathrm{p}<0.001$. 

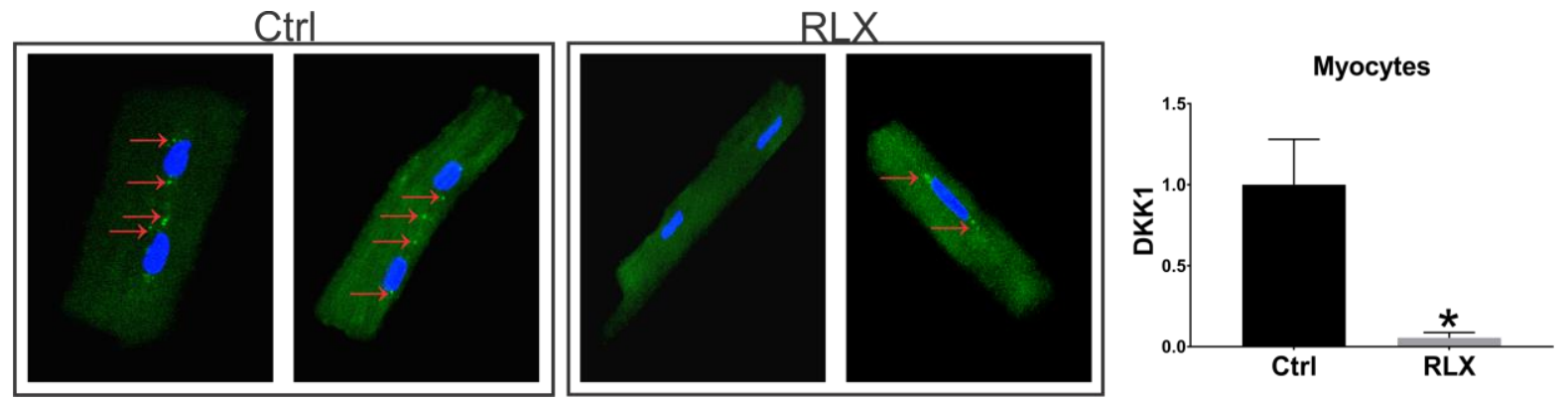

Figure 15: Relaxin Effects on Dikkopf-1 Expression: Isolated Cardiomyocytes. Relaxin treatment for 2-days significantly reduced expression of DKK1 in isolated cardiomyocytes compared to control cells ( $\mathrm{n} \geq 14$ cells/group). * indicates $\mathrm{p}<0.05$. 60x magnification.
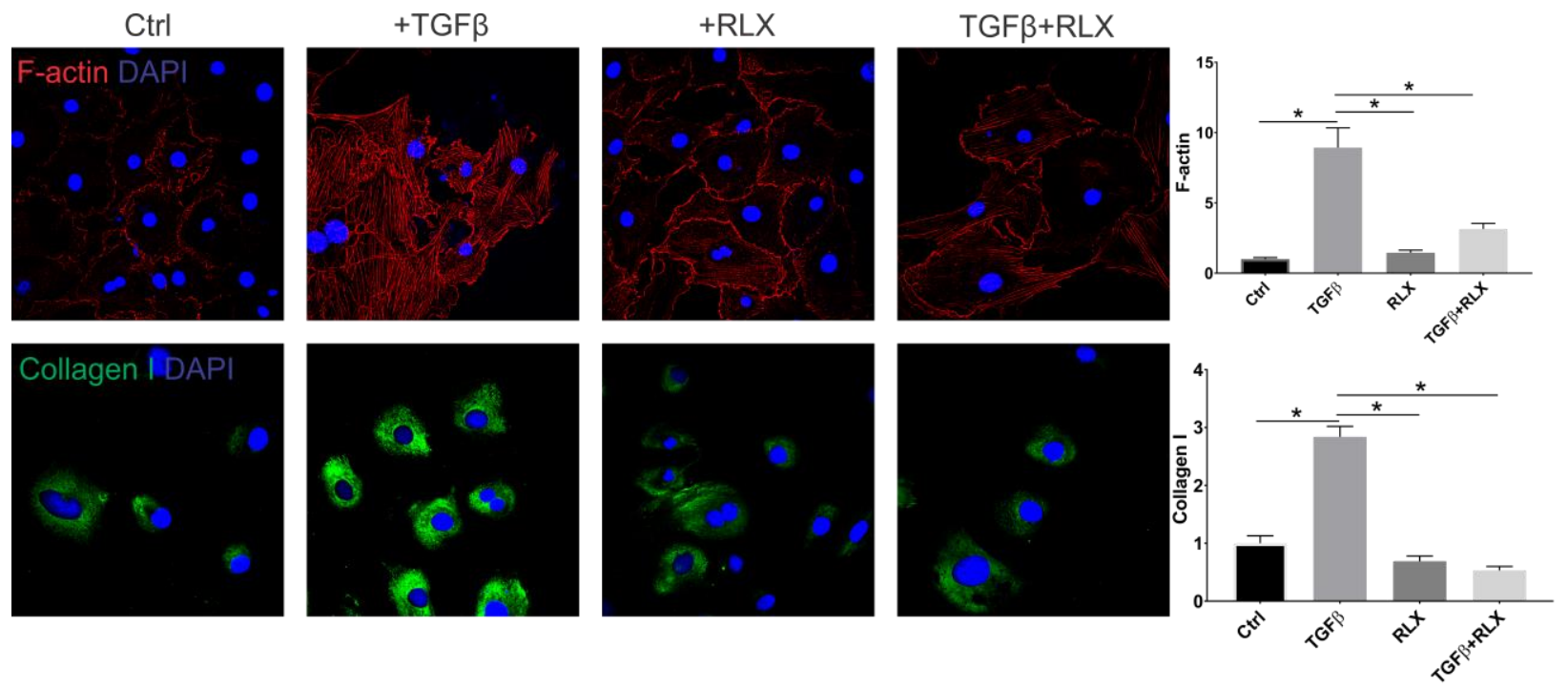

Figure 16. Relaxin Effects on Collagen Expression from Fibroblasts. Fibroblasts treated with TGF $\beta$ showed a marked increase in F-actin (top, $\mathrm{n} \geq 53$ cells/group) and collagen I (bottom, $\mathrm{n} \geq 41$ cells/group) expression that was dramatically inhibited by RLX. *indicates $\mathrm{p}<0.05$. $40 \mathrm{x}$ magnification. 

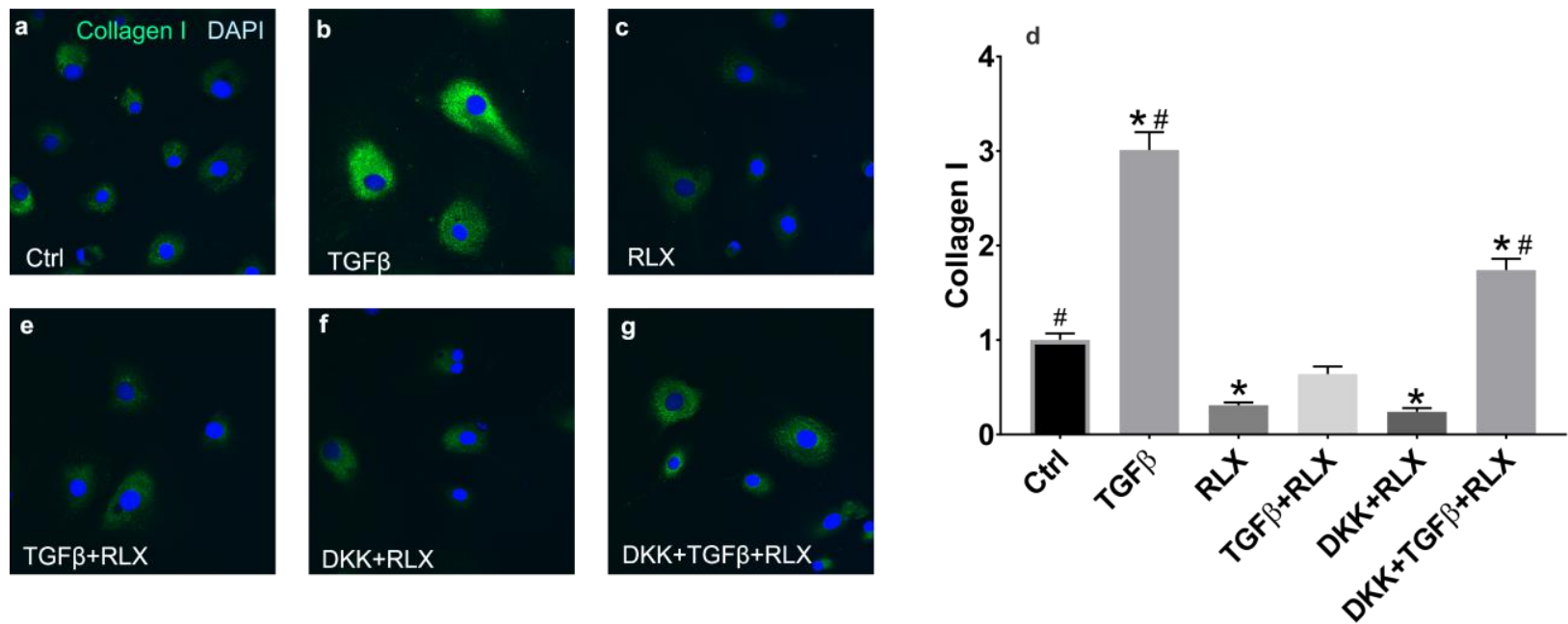

Figure 17. Relaxin-Wnt Interplay modulates Collagen Expression from Fibroblasts. Fibroblasts treated with TGF $\beta$ showed a 3 -fold increase in collagen expression that was dramatically inhibited by RLX to $31 \%$ of control expression (a-d). DKK1 blocked RLX's inhibitory effects of TGF $\beta$ mediated collagen expression ( $\mathrm{d}-\mathrm{g}) ; \mathrm{n} \geq 43$ cells/group. *indicates $\mathrm{p}<0.05$ compared to control. \# indicates $\mathrm{p}<0.05$ compared to RLX. $40 \mathrm{x}$ magnification.
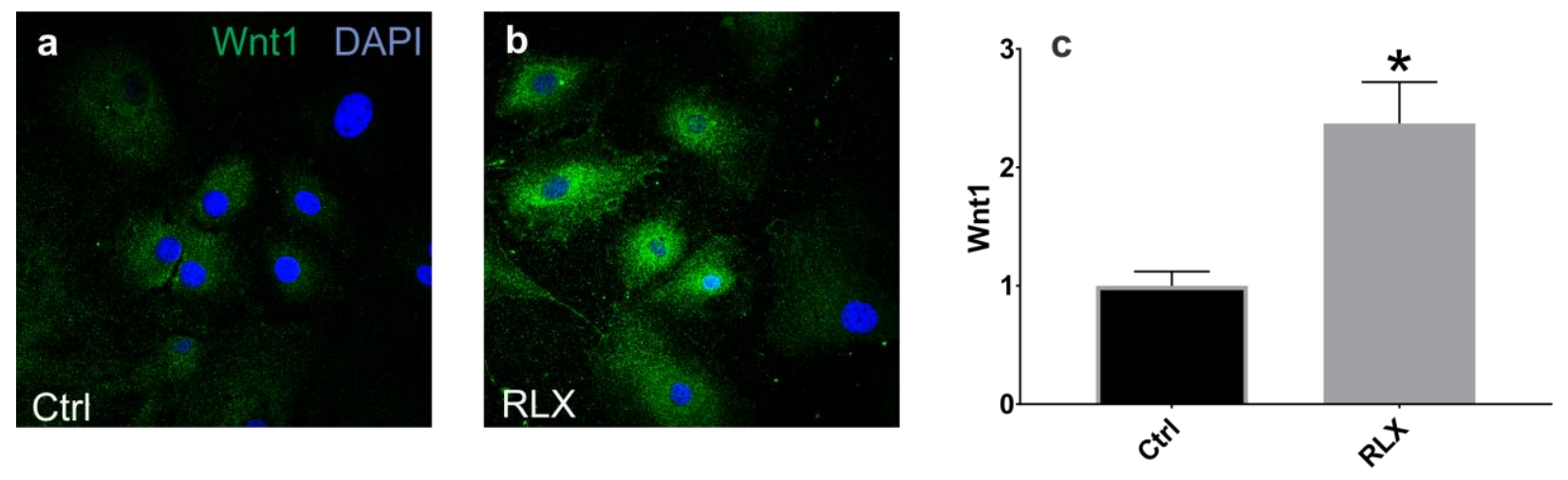

Figure 18. Relaxin Effects on Wnt1 Expression from Fibroblasts. Relaxin significantly increased expression of Wnt1 in cardiac fibroblasts. *indicates $\mathrm{p}<0.05 . \mathrm{n} \geq 46$ cells/group. 40x magnification. 
Based on intriguing clinical and pre-clinical data, RLX engendered significant enthusiasm as a potential therapy for cardiopulmonary diseases. In the acute heart failure trial RELAX-AHF, RLX treatment (30 $\mu \mathrm{g} / \mathrm{kg} /$ day for 2-days, i.v.) improved patient survival by a remarkable 37\% in 6 months.[109] These exciting results led the FDA to declare RLX as a "break-through" therapy made all the more significant because the trial included patients with systolic and diastolic HF. Unfortunately, the reduced mortality benefits were not duplicated in a subsequent larger clinical trial (www.novartis.com). Detailed analysis of the larger trial has not been reported and the failure of RLX to significantly reduce mortality is not fully understood. A possible explanation is that the control group of patients receiving standard of care for $\mathrm{HF}$ fared considerably better than in earlier trials but another problem has been the design methodology of a 2-days treatment which has justifiably received substantial criticism.[114] Our previous studies on the effects of RLX in experimental animals provide compelling evidence of significant beneficial effects of the hormone in cardiac physiology. We reported that RLX suppressed AF in aged (24-months old) rats by increasing conduction velocity (CV) of atrial action potentials.[13] These effects were linked to increased expression of the voltage-gated sodium channel (Nav1.5) and current (INa), and a marked decrease in fibrosis5, both effects confirmed here in ventricles. At the cellular level, Relaxin was shown to upregulate INa in 24-hours but the reversal of fibrosis required more than a week due to the slow turn-over of collagen in the extracellular matrix.[14] Besides electrical and ECM remodeling, we reported that Relaxin acted as a potent anti-immune and anti-inflammatory agent in the ventricles of aged animals.[8] Here we examine the mechanisms responsible for RLX's functions in the heart. Our results show that RLX's effects are largely mediated by the modulation of canonical Wnt signaling which can act as a master controller of gene expression in heart and other organs. 
This conclusion is based on several major lines of evidence: a) GSK3 $\beta$ inhibitors and exogenous Wnt1 protein mimic the effects of RLX in cardiomyocytes; b) recombinant Dkk1, a specific natural inhibitor of canonical Wnt signaling, blocks the effects of RLX on Nav1.5 and collagen I expression; c) Dkk1 expression is reduced by RLX in LV tissue and isolated cardiomyocytes; and d) RLX increases the expression of the Wnt1 ligand in cardiac fibroblasts. The integration of RLX and Wnt signaling was previously suggested by studies in prostate cancer18, but the mechanisms described here are very different. Whereas in prostate cancer RLX promotes tumor progression via its effects on the protocadherin PCDHY [184], the effects we described are mediated, at least in part, by increased expression of Wnt1 and reduced expression of Dkk1.

Wnt signaling in the heart is complex, and different Wnt ligands have distinct effects. Previous studies of the actions of Wnt-signaling focused on isolated neonate rat ventricular myocytes (NRVM) and non-cardiac fibroblasts. In these earlier studies, treatment with Wnt1 and inhibition of GSK3 $\beta$ increased Cx43 expression and trafficking to the ID [81,84], results that are consistent with those reported here. The role of Wnt signaling in the regulation of Nav1.5 is more controversial. Liang et al. reported that incubation of NRVM with Wnt3a or CHIR-99021 down-regulated Nav1.5 [82], results that were confirmed using the immortalized cardiomyocyte cell line HL-1 [182]. In contrast, findings by Asimaki et al [182], Chelko et al [84] and data presented here, show that canonical Wnt signaling and GSK3 $\beta$ inhibition increase Nav1.5 expression in adult, aged, and diseased cardiomyocytes, suggesting that, like Wnt-signaling, RLX signaling is highly dependent on cell type and age. 


\subsection{Relaxin As A Therapy For PAH}

Pulmonary arterial hypertension (PAH) is a progressive disease of the pulmonary vasculature which primarily affects the small and intermediate pulmonary arterioles. PAH is the result of a complex pathological process which consists of elements of endothelial dysfunction, increased pulmonary artery contractility, proliferation and remodeling of endothelial and smooth muscle cells and in situ thrombosis.[189] The pathological processes lead to progressive narrowing of the pulmonary arterioles, resistance to blood flow and an increase in PA pressures resulting in pulmonary hypertension.[190] The etiology of PAH can be idiopathic, genetic, associated with other medical conditions, drug-induced or a consequence of pulmonary venoocclusive disease. PAH is a specific classification within the broader disease of pulmonary hypertension which can also be secondary to left ventricular heart failure, chronic lung disease, chronic thromboembolic disease or other unclear/multifactorial mechanisms.[191] As PAH progresses, there is an increase in pulmonary vascular resistance and afterload, and the right ventricle (RV) begins to undergo compensatory hypertrophy and fibrosis to maintain systolic function and cardiac output. Eventually, these compensatory mechanisms fail in the face of chronically escalating pulmonary pressures leading to RV dilation and failure. Sudden death can occur in severe RV failure resulting from inadequate left ventricular (LV) filling, loss of systemic blood flow, followed by cardiac asystole.[190] 
Although cardiac arrhythmias play an important role in the morbidity and mortality of PAH patients, [192] little is known about the arrhythmia phenotypes in the RV, especially compared to existing knowledge of the nature and structure of arrhythmias in LV dysfunction and disease.[125, 193-197] Right-sided cardiac remodeling in response to chronic pressure and volume overload produce an arrhythmogenic substrate in patients with PAH. Factors contributing to arrhythmogenic remodeling include, modulation in autonomic activity, delayed cardiac repolarization and RV myocardial ischemia.[192, 198, 199] Given the paucity of data and understanding of RV arrhythmogenicity in PAH, we investigated the electrophysiological properties of the RV in an animal model of PAH. The sugen-hypoxia (SuHx) rat model of PAH creates sustained severe pulmonary hypertension which is accompanied by the formation of intrapulmonary plexiform lesions with many histopathological features of pulmonary arteriopathy seen in human PAH.[200, 201] Although this model recapitulates the histological and hemodynamic effects of PAH, it has not been characterized from an electrophysiological perspective. In addition, the model offers a unique opportunity to explore the effects of putative therapies for PAH by measuring changes in the histology and hemodynamics of the SuHx rat.

Relaxin (RLX) is a 53 amino acid pleiotropic hormone first characterized for its role in reproduction and pregnancy, but has more recently been shown to act in multiple organ systems, including the heart.[202] The effects of RLX on pulmonary fibrosis was previously investigated in rat hypoxia and murine bleomycin models of pulmonary hypertension.[61,203] In both models, RLX-treatment was found to reverse pulmonary fibrosis, collagen deposition and alveolar thickening.[61,203] In RLX deficient mice $\left(R L X^{-/}\right)$, there is an age-dependent progression of lung fibrosis associated with increased tissue weight, collagen content and alveolar congestion which were reversed by treating $R L X^{-/}$mice with exogenous relaxin.[204] In 
addition, relaxin has been shown to increase arterial compliance, cardiac output and renal blood flow.[153,205,206] Moreover, our group previously showed that RLX suppresses atrial fibrillation (AF) and reverses fibrosis in spontaneously hypertensive rats (SHR) [14] and in aged (24 months-old F-344 strain) rats.[13] Relaxin suppressed AF by increasing atrial conduction velocity through reversal of fibrosis and cellular hypertrophy, increasing $\mathrm{Na}^{+}$channel expression, $\mathrm{Na}^{+}$current density and connexin-43 phosphorylation. Importantly, human cardiomyocytes from induced pluripotent stem cells treated with RLX showed a 2-fold increase in $\mathrm{Na}^{+}$current density.

The beneficial cardiovascular effects of RLX formed the rationale for human trials, which have met with mixed results. Phase 1 and 2 trials achieved a primary endpoint of greater dyspnea relief but more interestingly, there was a significant decrease in mortality at 6 months, primarily due to reductions in stroke and sudden cardiac death, $[110,112]$ though a recent trial focused on reduced mortality failed to achieve its primary end points (https://www.novartis.com/ News Release of March 2017).

Given the promising data in clinical and pre-clinical studies, we now examine the ability of RLX to ameliorate the deleterious cardiac effects of SuHx-induced PAH on the heart. We demonstrate that RLX treatment suppressed RV arrhythmias and asystole which are typically observed in PAH rats. The protective effects were primarily due to an increase in $\mathrm{CV}$, pulmonary compliance, reduction of pulmonary occlusion of pre-artery vessels and decreases in cardiac and inter-vascular lung fibrosis. These data suggest that RLX may improve survival of PAH patients.

\section{Animal Protocols}

Figure 19 summarizes the experimental protocol; starting with in situ Doppler-flow measurements, isolated Langendorff perfused hearts for arrhythmia phenotype measurements by optical mapping and ending with histology of cardiac and lung tissues. 


\section{Experimental Design}

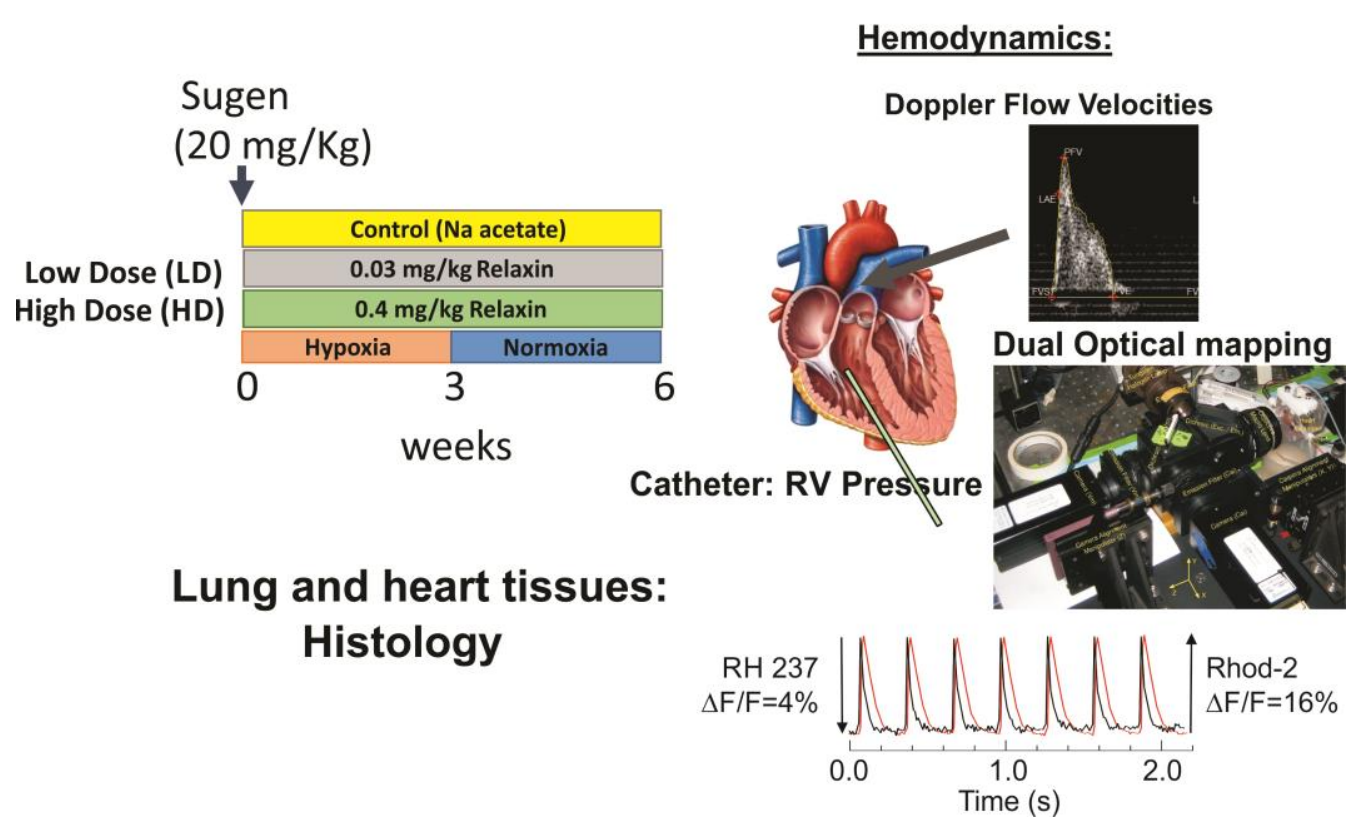

Figure 19. Experimental Protocol of Pulmonary Arterial Hypertension Study. Rats were treated with Sugen (20 mg/kg) on day 0 and were placed in a hypobaric chamber (hypoxia: $10 \%$ oxygen) for 3 -weeks then in $21 \%$ oxygen (normoxia) for another 3 -weeks. Rats were implanted with mini-osmotic pumps to deliver $\mathrm{Na}$ acetate $(\mathrm{SuHx})$, a low $(0.03 \mathrm{mg} / \mathrm{kg} / \mathrm{day})$ or a high $(0.4 \mathrm{mg} / \mathrm{kg} / \mathrm{day}) \mathrm{dose}$ of Relaxin. The three groups: SuHx treated rats with Na-acetate, low dose (LD) or high dose (HD) of Relaxin treatment were also compared to control non-PAH rats that did not receive the SuHx treatment. At the end of the 6-weeks, pressure and Doppler flow catheters were used to measure hemodynamics in anesthetized rats followed by Langendorff perfusion of hearts for optical mapping of the arrhythmia phenotype and isolation of lung and heart tissues for histology.

\section{Sugen-hypoxia PAH in Rats}

Rats treated with SuHX develop pulmonary arterial hypertension as evident by a significant increase in RV hypertrophy (Fulton Index), RV to Tibia length ratio, systolic RV pressure and pulmonary vascular resistance compared to controls (not shown). There were no significant differences in heart rate between the groups (not shown). Neither RLX LD or RLX HD had any significant effect on pulmonary pressure or RV hypertrophy. (Figure 20). Flow 
velocity profiles in rats treated with SuHx had mid-systolic notching indicative of significant pulmonary vascular remodeling (Figure 20A, n=6 hearts/group). The notching pattern was reduced in rats treated with a high dose but not a low dose of RLX. Heart rate did not significantly change among the 4 groups (Figure 20B). SuHx rats had no significant changes in mean velocity, stroke distance, cardiac output or stroke volume compared to controls (Figure 20C-F). In the rats treated with RLX LD, there was a significant decrease in mean velocity, stroke distance, cardiac output and stroke volume compared to the rats treated with RLX HD.

\section{Arrhythmia Vulnerability}

After catheterization, hearts were explanted and perfused for optical mapping of APs and CaTs. The intrinsic heart rate was monitored under sinus rhythm followed by programmed stimulation to test the arrhythmia phenotype. All control hearts $(n=9)$ were in sinus rhythm and no spontaneous arrhythmias or ectopic beats were noted. Simultaneous recordings of APs and CaTs were similar among the 4 groups (Control, SuHx, SuHx+ RLX LD and SuHx + RLX HD) during sinus rhythm with no observable dysfunction in CaTs (not shown). Control, SuHx and SuHx + RLX treated hearts underwent programmed stimulation to evaluate their arrhythmia vulnerability. In control hearts, pro-arrhythmic pacing induced a sustained VF in 1 out of 9 hearts. In SuHx PAH hearts (n=11), two types of electrical dysfunctions were observed. 1) One phenotype of SuHx hearts $(n=4 / 11)$ had severe bradycardia relative to controls (SuHx heart rate was $23 \pm 31$ versus controls $137 \pm 48$ beats per minute, $\mathrm{p}=0.001$ ) (Figure 21Ai). These hearts could be paced at faster rates for shorts intervals (5-10 s) and became asystolic. Electrical stimulation of hearts in asystole failed to capture or elicit APs and CaTs (Figure 21ii-iii). 2) The

second phenotype of SuHx hearts had normal heart rates and spontaneous episodes of VF of 
various durations $(2-50 \mathrm{~s})(\mathrm{n}=5 / 11)$ that progressed to sustained VF, (see supplemental movie) $(n=3 / 11)$ (Figure 21B).

Treatment of SuHx PAH rats with RLX LD ( $n=8)$ abrogated both types of electrical dysfunctions: 1) sinus bradycardia and arrest and 2) spontaneous self-terminating VF and sustained VF. As shown in Figure 21C, programmed stimulation with premature impulses at S1$\mathrm{S} 2=85$ and $70 \mathrm{~ms}$ (Figure 21Ci, ii) did not elicit arrhythmias and burst pacing elicited a few beats of transient VF ( $\mathrm{n}=4 / 8)$ before recovering to sinus rhythm (Figure 21Ciii). In rats treated with RLX HD ( $\mathrm{n}=8)$, programmed stimulation with $\mathrm{S} 1-\mathrm{S} 2=60$ and $55 \mathrm{~ms}$ failed to induce an arrhythmia (n=0/8; Figure 21Di-ii) and burst pacing also failed to elicit even a brief arrhythmia (Figure 21Diii, n=1/8). Thus, RLX HD rescued PAH hearts from bradycardia and asystole as well as sustained arrhythmias elicited either spontaneously, by programmed stimulation or by burst pacing $(\mathrm{n}=0 / 8)$. Figure $21 \mathrm{E}$ and $\mathrm{D}$ summarize the arrhythmia phenotype observed in PAH hearts without and with treatments with RLX LD or HD.

Besides RLX treatment, several pharmacological and ionic interventions were attempted to rescue PAH hearts from arrest. All failed except for adding dithiothreitol (DTT =1 mM) to the perfusate. Figure 22A illustrates a voltage (blue) and a calcium (red) signal from a pixel on the RV of a PAH heart. Pacing the heart failed to elicit APs and CaTs despite testing a wide range of stimulation conditions and a rare spontaneous doublet of depolarization occurred which suggests a potentially functional RV (Figure 22A). 


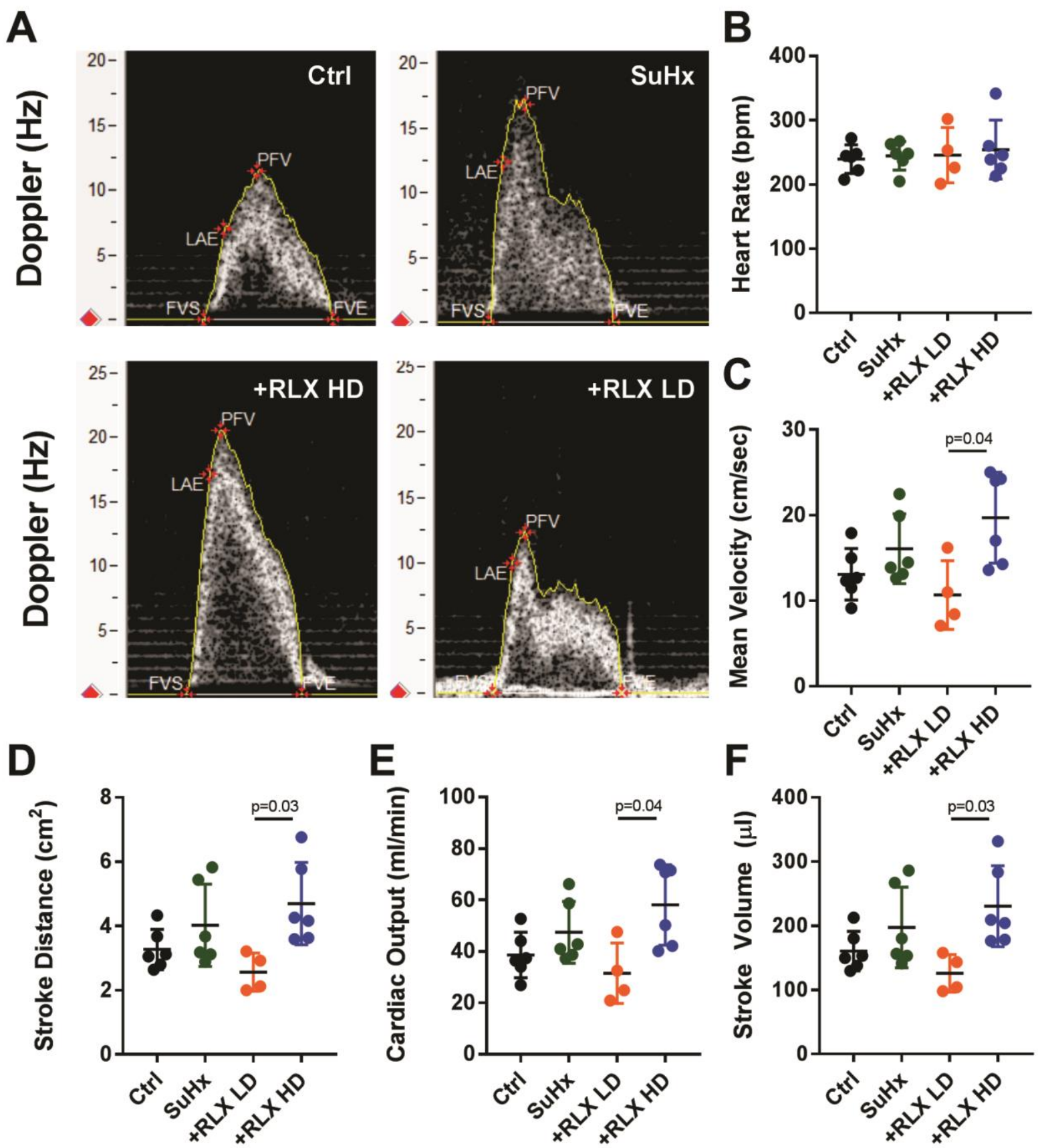

Figure 20. Relaxin/Sugen Effects on Doppler Flow Patterns. A) Representative Doppler flow velocities at the right ventricular outflow track show significant midsystolic notching in SuHx rats compared to controls (Ctrl). A high dose of Relaxin (+RLX HD) reduced the notching where there was no effect of the low dose of Relaxin (+RLX LD). B) All Doppler measurements were taken at similar heart rates. SuHx rats had no significant changes in C) mean Velocity, D) Stroke Distance, E) Cardiac Output or F) Stroke Volume compared to controls. A high dose of Relaxin significantly increased mean velocity, stroke distance, cardiac output and stroke volume compared to a low dose of Relaxin. 
A.

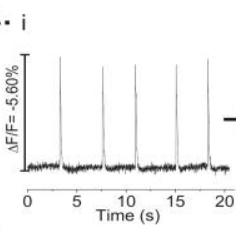

ii

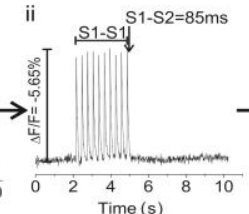

iii

B.

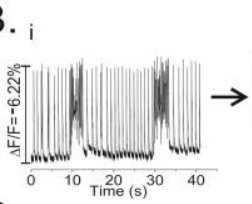

C.

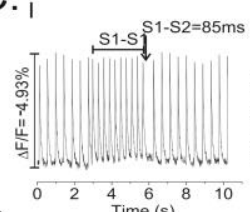

D.

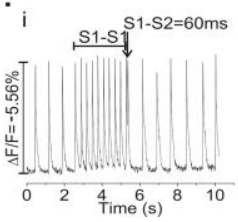

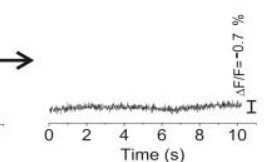

iii

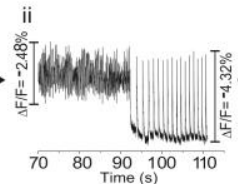

ii
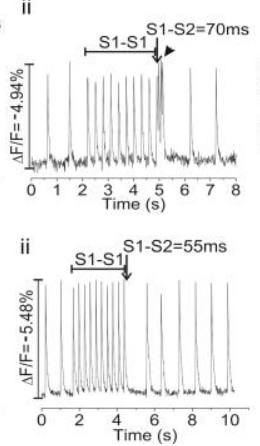

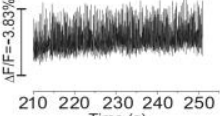

iii Non-Sustained

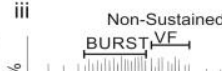

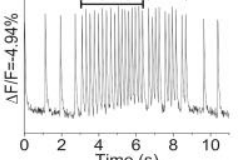

iii

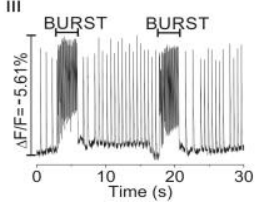

E.

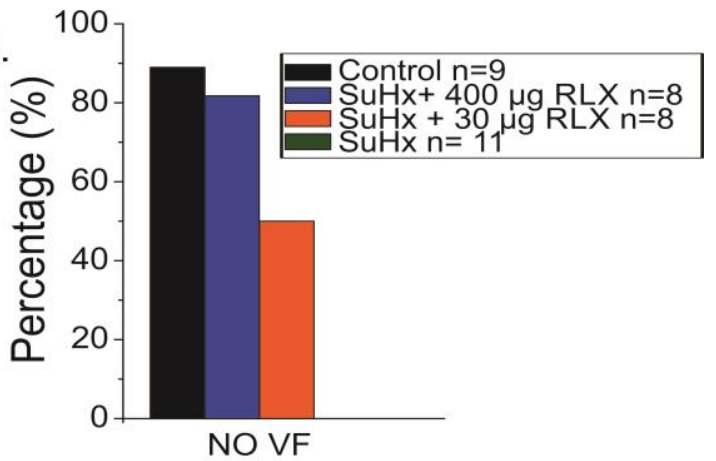

F.

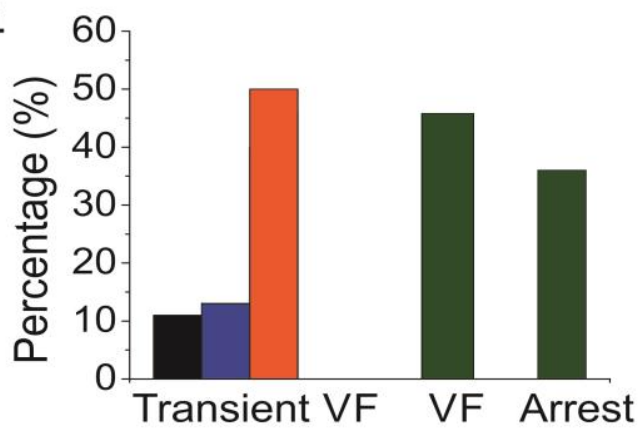

Figure 21. Arrhythmia Phenotypes of Pulmonary Arterial Hypertension Hearts. Optical mapping of SuHx hearts was used to measure RV and LV action potentials (APs) which revealed two equally prevalent phenotypes. A: SuHx hearts were in bradycardia (i) and could be briefly paced at higher rates (ii) which was followed by cardiac arrest (iii) (n=4/11). Once in cardiac arrest, electrical stimuli of various amplitude (10X threshold) and duration (1-10ms) failed to capture of elicit an AP. B: The other SuHx hearts exhibited spontaneous ventricular fibrillations (VF) (i) which increased in durations, were self-terminating (ii) and transitioned to a sustained VF, in this illustration in $\sim 3 \mathrm{~min})$ (iii) $(\mathrm{n}=5 / 11) \mathrm{C}$ : Relaxin at low dose suppressed sustained VF and cardiac arrest $(n=0 / 8)$. Panel (i) illustrates AP recordings in sinus rhythm, followed by $2 \mathrm{~s}$ of rapid pacing and a premature impulse at S1-S2 of $85 \mathrm{~ms}$ which failed to elicit a VF. Panel (ii) illustrates another SuHx +RLX LD heart under bradycardia, followed by rapid pacing and a premature impulse $(\mathrm{S} 1-\mathrm{S} 2=70 \mathrm{~ms})$. Here, the premature impulse elicits a brief transient arrhythmia (4 beats) and a return to sinus rhythm. Panel (iii) shows that burst pacing (4 s) elicits a transient VF that self terminates in < 3s). D: RLX at high dose suppressed cardiac arrest and burst pacing-induced VF Panels (i-ii) show that rapid pacing and premature impulses of S1-S2 $=60$ or $55 \mathrm{~ms}$ failed to elicit VF and HD RLX suppressed al VF even after repeated burst-pacing events (panel (iii). E: Histogram of the 4 groups of hearts resistant to VF after rapid pacing, programmed stimulation and burst pacing. F: Summary of the 4 groups of hearts that exhibited transient and sustained VF or cardiac arrest. 
A. SuHx Asystole: failure to capture during stimulation
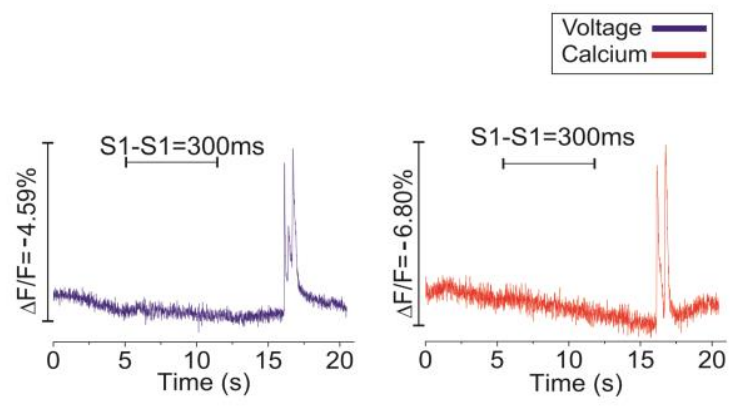

C. SuHx Arrhythmogenic phenotype in Sinus Rhythm
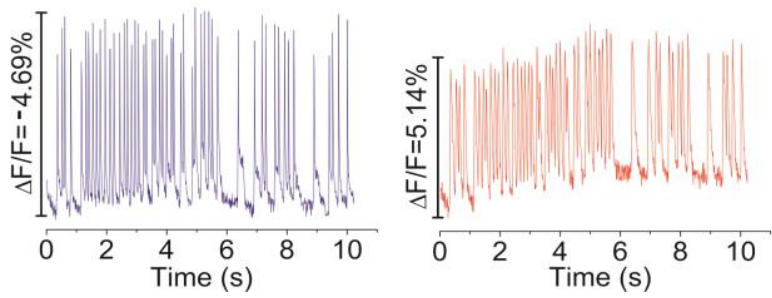

B. SuHx Asystole + $1 \mathrm{mM}$ DTT:

rescues capture during stimulation
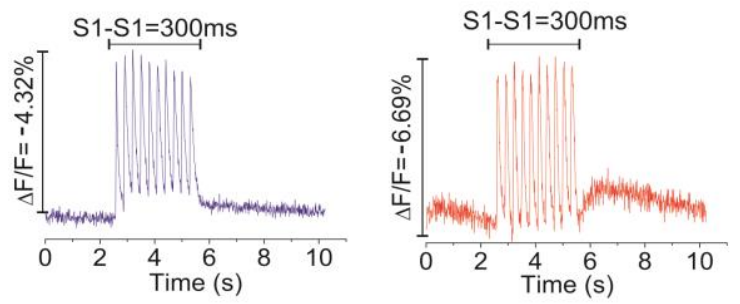

D. SuHx: $1 \mathrm{mM}$ DTT rescues Arrhythmogenic phenotype

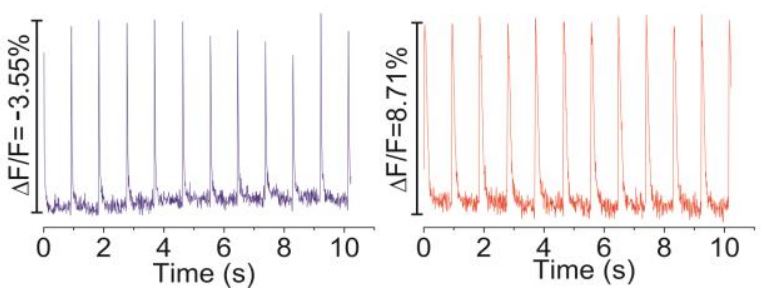

Figure 22. Dithiothreitol Rescues Asystolic Pulmonary Arterial Hypertension Hearts. A: Dual optical mapping of voltage (blue) and $\mathrm{Ca}^{2+}$ (red) of an asystolic SuHx heart shows that the heart has no sinus rhythm and fails to capture during pacing at S1-S1 = 300 ms. A rare spontaneous salvo of depolarization self-terminates but the heart remains in-excitable. B: The same heart as in (A) is rescued by perfusing the heart with $1 \mathrm{mM}$ DTT $(2-3 \mathrm{~min})$, as an electrical impulse successfully capture at every beat $(\mathrm{n}=2 / 2$ hearts). C: Voltage and Calcium mapping of a SuHx heart in sinus rhythm illustrates spontaneous bursts of tachycardia which typically progress to sustained VF. D: Perfusion of the same heart with DTT $(1 \mathrm{mM})$ stabilized the intrinsic sinus rhythm for the remainder of the experiment $(\mathrm{n}=2 / 2)$. 
The same heart was then perfused with DTT (1 $\mathrm{mM}$ for $5 \mathrm{~min}$ ) and electrical stimuli (300 ms $\mathrm{CL}$ ) resulted in capture and propagation of APs and CaTs (Figure 22B, $n=2 / 2$ ). DTT rescued the other arrhythmogenic phenotype observed in PAH hearts. Figure 22C illustrates a PAH heart that exhibits spontaneous transient VF that typically progresses to sustained VF; however, perfusion with DTT (1 mM) suppressed these arrhythmias resulting in a recovery to a stable sinus rhythm $(n=2 / 2)($ Figure 22D).

\section{Conduction Velocities (CV) and Restitution Kinetics of CV}

In two models of atrial fibrillation (AF), relaxin suppressed AF in part by increasing $\mathrm{CV}$ in the atria. $[22,23]$ Here, CV was measured from the epicardium of the RV and LV by pacing each ventricle separately at $300 \mathrm{~ms}$ CL. The longitudinal CV of control hearts was $0.84 \pm 0.2 \mathrm{~m} / \mathrm{s}$ in both the RV and LV $(n \geq 4)$ and was markedly reduced in PAH hearts by 2.2 -fold relative to controls (RV: $0.39 \pm 0.14 \mathrm{~m} / \mathrm{s}, \mathrm{LV}: 0.38 \pm 0.11 \mathrm{~m} / \mathrm{s} ; \mathrm{n} \geq 4$ ) (Figure 23A). Low-dose of RLX partially reversed CVs (RV: $0.53 \pm 0.15 \mathrm{~m} / \mathrm{s}$ and LV: $0.62 \pm 0.12 \mathrm{~m} / \mathrm{s}, \mathrm{n} \geq 4 /$ group) and the RLX HD increased RV and LV CVs by 1.9 and 2.0-fold $(\mathrm{n} \geq 4)$. PAH hearts that received RLX HD had CVs that were not statistically different from control hearts (RV: $0.79 \pm 0.21 \mathrm{~m} / \mathrm{s}, \mathrm{LV}: 0.73 \pm 0.08$ $\mathrm{m} / \mathrm{s}$ ). CVs in the RVs of the RLX LD group were statistically slower than in the RV of the RLX HD group ( $\mathrm{p}=0.02)$ whereas there was a trend towards slower CVs in the LVs $(\mathrm{p}=0.09)$. These data are consistent with previous findings in atria and show that RLX increases CVs in a dosedependent manner.

Restitution kinetics (RK) of CV was measured by applying 20 paced impulses (S1) with $\mathrm{S} 1-\mathrm{S} 1=300 \mathrm{~ms}$ followed by a single premature impulse (S2) at variable S1-S2 delays (Figure 23B). High-dose of RLX treatment significantly improved RK of CV relative to SuHx, and RLX 
LD improved CV but was less effective (Figure 23B). Thus, PAH results in a marked suppression of CV at all cycle lengths as well as the RK of CV and RLX-treatment reversed this arrhythmogenic effect in a dose-dependent manner. SuHx prolonged AP durations (APDs) measured at $\mathrm{APD}_{75}$ and RLX HD reversed APD prolongation (not shown) but these tendencies did not reach statistical significance. Similarly, the restitution kinetics of $\mathrm{APD}_{75}$ was not statistically altered in the 4 groups (not shown).

\section{Ventricular Remodeling}

In the SuHx model of PAH, the RV underwent a remodeling of the extracellular matrix as an increase in fibrosis in response to the increase in pulmonary artery pressure. In PAH compared to control hearts, the collagen to tissue ratio in the RV and LV increased by 3.4 and 3.5-fold, respectively (Figure 24A). When SuHx rats were concomitantly treated with RLX LD, there was no change in RV fibrosis but a $40 \%$ decrease in $\mathrm{LV}$ collagen content ( $\mathrm{n} \geq 4)$. When SuHx rats were treated with the RLX HD, RV and LV collagen were reduced 45 and $47 \%$, respectively ( $\mathrm{n} \geq 4$ ). Representative images taken from all 4 groups from the RV and $L V$ are shown in Figure 24A. Figure 24B compares the differences in fibrosis observed in the RV and LV in control and PAH hearts and how they were altered by RLX LD or HD, showing the RV contained significantly more collagen than the LV as is expected in PAH. 


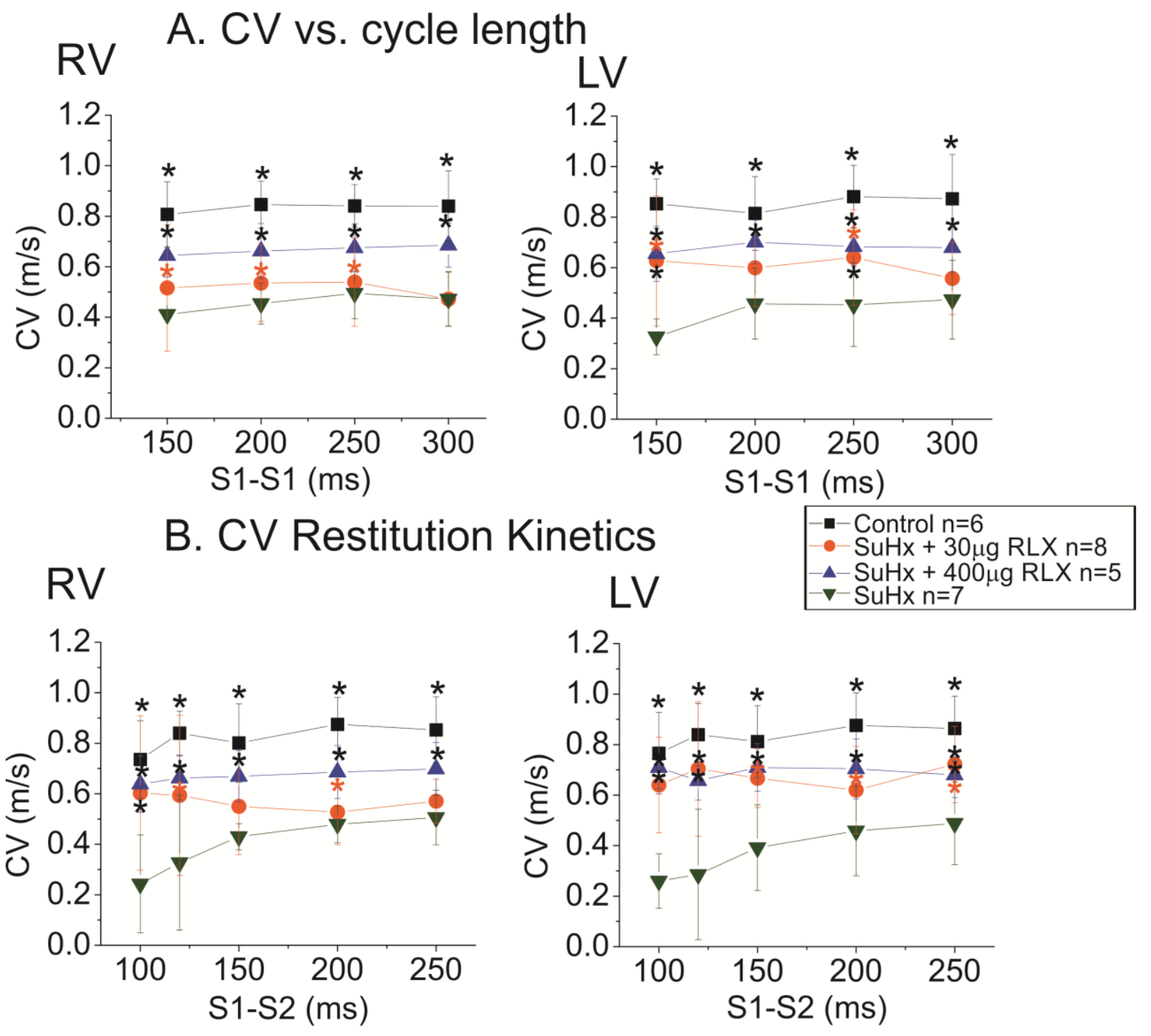

Figure 23: Relaxin Effects on Conduction Velocity in Pulmonary Arterial Hypertension. A: Plots of CV vs. cycle length show a $50 \%$ decrease in CV in the RV (left) and LV (right) of SuHx hearts compared to controls. RLX treatments partially reverse the decrease of $\mathrm{CV}$ in a dose-dependent manner. $\mathrm{B}$ : The restitution kinetics (RK) plots the CV of the premature $\mathrm{AP}$ as a function of S1-S2 inter-pulse interval. CV RK curves of SuHx hearts have a steeper slope and are reduced compared to controls in the RV and LV and RLX reduces the slope of CV RK curves and increase CVs in a dose-dependent manner. * indicates $\mathrm{p}<0.05$ vs. SuHx. 

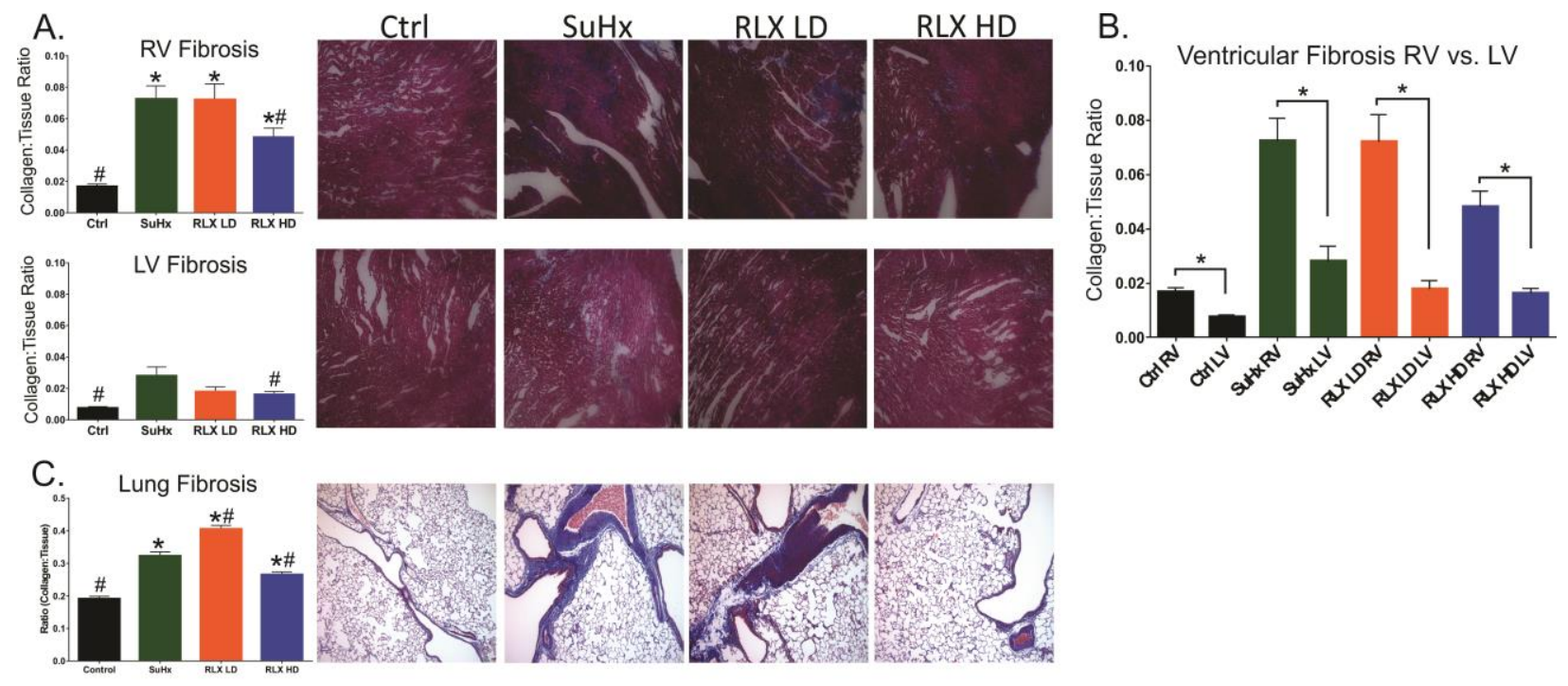

Figure 24: Relaxin Effects on Ventricular and Pulmonary Fibrosis. A: Top. RV collagen to tissue ratio for control, SuHx, SuHx + RLX LD or +RLX HD rats. Collagen to tissue ratio increased $\sim$-fold in SuHx compared to control rats, did not significantly change with LD RLX and decreased by $40 \%$ in HD RLX ( $>4$ per group). Bottom. LV collagen to tissue ratio for control, SuHx, SuHx $+\mathrm{LD}$ RLX and SuHx $+\mathrm{HD}$ RLX rats. Collagen to tissue ration increased 4-fold in SuHx compared to control and decreased by $\sim 50 \%$ in SuHx + LD and HD RLX ( $>4$ per group). Sections shown at 10x magnification, tissue stained with Mason's Trichrome. Blue: collagen; Purple: myocardial tissue. * indicates $\mathrm{p}<0.05$ vs. control. \# indicates $\mathrm{p}<0.05$ vs. SuHx. B: Comparisons show that fibrosis was more elevated in the RV than the LV. C. Top panels illustrate trichrome histological sections to visualize peri-vascular fibrosis in control, SuHx, LD RLX and HD RLX treated rats. Analysis of bilateral lung sections ( $\mathrm{n} \geq 4$ rats per group) show a $\sim 2$-fold increase in fibrosis in SuHx compared to controls, an unexplained small but significant increase in fibrosis with RLX LD and a 15\% decrease in HD RLX compared to SuHx. Blue: collagen. * indicates p<0.05 vs. control. \# indicates p<0.05 vs. SuHx. 


\section{Pulmonary Vasculature}

Figure 24C shows that PAH caused the expected increase in perivascular fibrosis which increased by 1.6 -fold ( $\mathrm{n} \geq 4$ ) and was not significantly lowered RLX LD whereas the RLX HD significantly reversed the fibrotic insult from 1.6 to 1.4 -fold ( $\mathrm{n} \geq 4$ ).

Based on smooth muscle actin and trichrome staining, there was respectively significant muscularization of the vessels and peri-vascular fibrosis in PAH (Figure 25, panel $J$ and $L$ ) compared to control (panel I and $K$ ) lungs. Plexiform lesions (panel G), disintegration of the lamina interna (panel F) and occlusion (panel E) of small pulmonary arteries are prominently observed in PAH lungs and were absent in controls (panels $A$ and $B$ ) and were moderately reduced in RLX-treated PAH lungs (panels $C$ and $D$ ).

PAH lungs had fewer 'open’ pulmonary vessels $68 \%$ compared to $100 \%$ in controls and RLX (LD and HD) increased the percentage of 'open' vessels to $60 \%$ and $65 \%$, respectively, though this did not reach significance (Figure 26A, left panel). Low and high doses of RLXtreatment reduced the percent of partially occluded pulmonary vessels compared to untreated PAH rats (middle panel) and the percentage of occluded vessels in PAH lungs did not significantly change with RLX LD or HD (right panel). Notably in PAH patients and SuHx rats, intima or media fractional thickness and occlusive lesions, but not number of plexiform lesions, correlate with pulmonary artery pressure.[207,208] Figure 26B shows that the percent of vessels with plexiform lesions was zero in controls, $8 \%$ in $\mathrm{Su}-\mathrm{Hx}$ and did not significantly decrease to $7 \%$ and 5\% at RLX LD, respectively (left panel). The high-dose of RLX reduced the number of plexiform lesions/slide compared to untreated PAH rats (middle panel), but did not reach significance, and approximately the same number of vessels were examined in all 4 groups (right panel).[207, 209] 


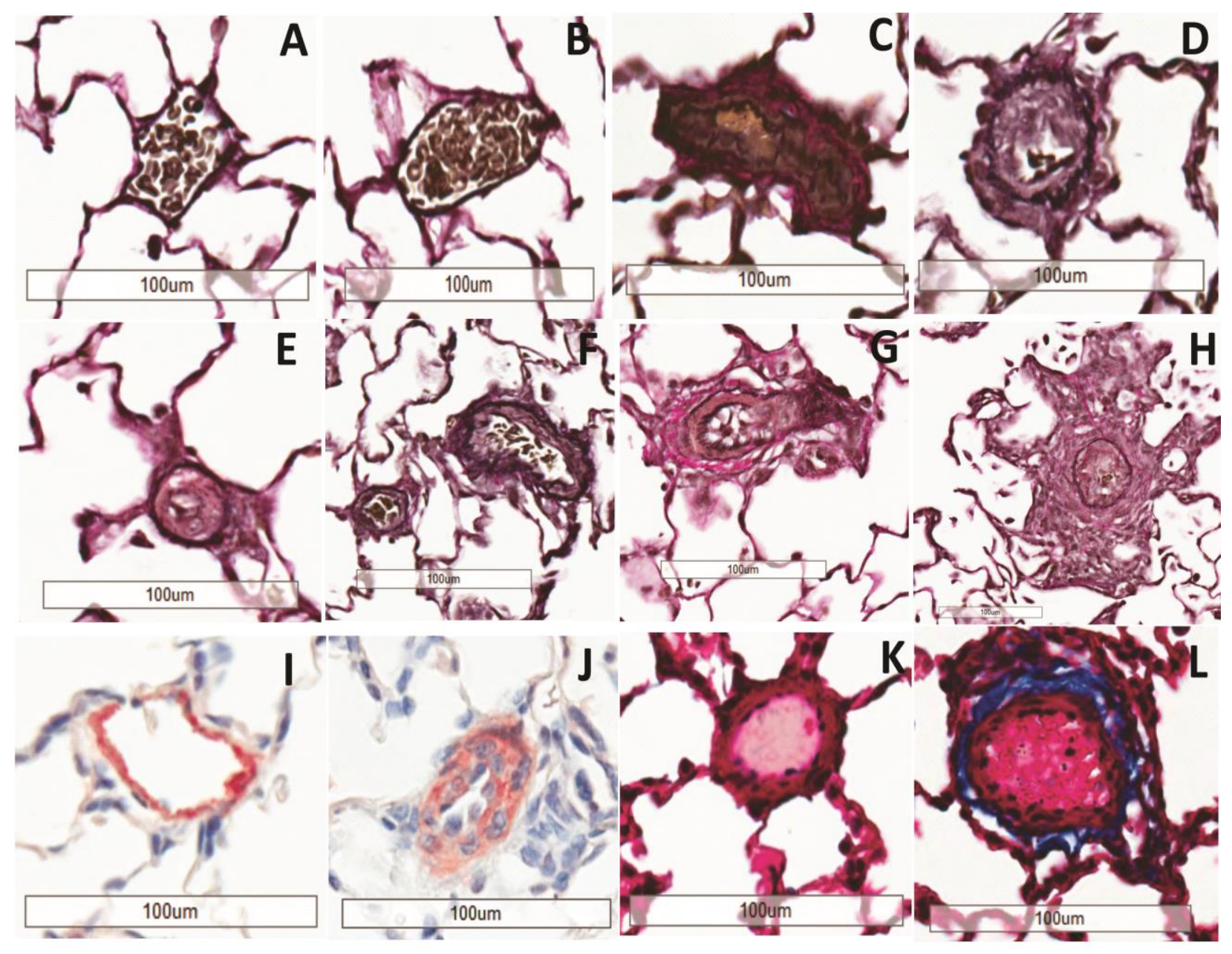

Figure 25: Histology of Diseased and Relaxin Treated Lungs. A \& B: Pre-capillary pulmonary vessels in control animals. C \& D: partially or E: completely occluded vessels in SuHx rats. F \& G: early disintegration of lamina interna and plexiform lesion in G from lung of SuHx rat. I: minimal muscularization and $\mathrm{K}$ : absence of perivascular fibrosis in control rat. J: muscularization and L: perivascular fibrosis in SuHx rat. Verhoeff's Van Gieson (A-H), smooth muscle-alpha actin (I, J) and trichrome (K, L) staining. 


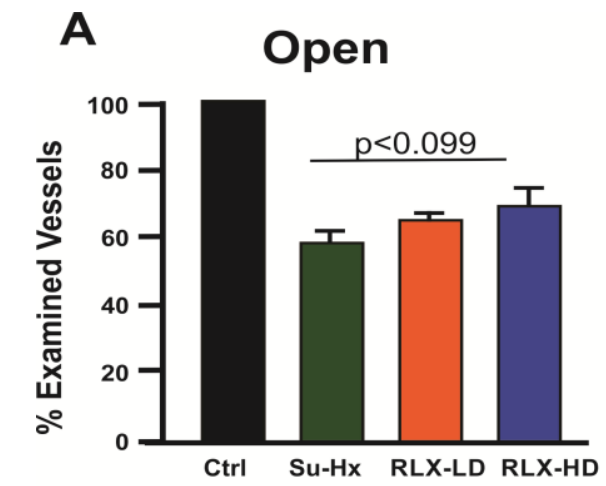

Partially Occluded

Occluded
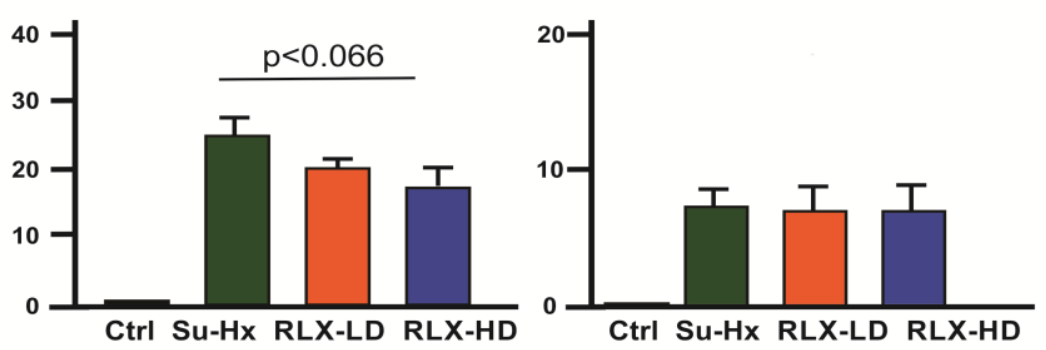

B Plexiform Lesions
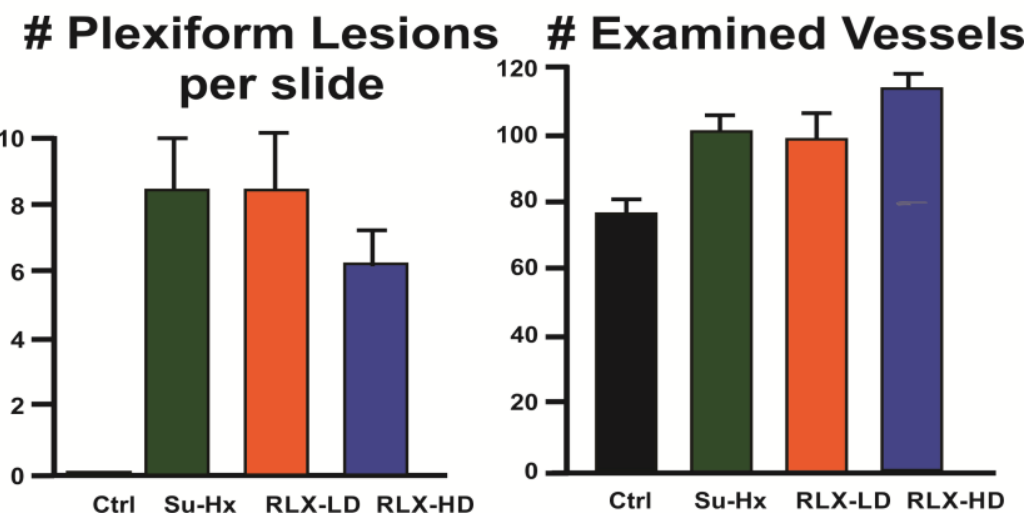

Figure 26. Occlusion and Plexiform Lesion Quantification of Diseased and Relaxin Treated Lungs. Quantification of occlusive and plexiform (PLX) lesions in male Sprague Dawley rats treated with Sugen $5416(20 \mathrm{mg} / \mathrm{kg})$ and exposed to hypoxia for 3 weeks, followed by normoxia for 3 weeks $(\mathrm{Su}+\mathrm{Hx})$ or $\mathrm{Su}+\mathrm{Hx}$ rats treated with relaxin. 
In this SuHx model of pulmonary hypertension, there was a significant increase in systolic RV pressure with compensatory remodeling including RV hypertrophy and fibrosis. Optical mapping studies identified a marked decrease in CV and two striking electrical phenotypes in untreated PAH rats, a) bradycardia followed by cardiac arrest and b) spontaneous VF that were self-terminating or progressed to sustained VF. RLX-treatment produced a dosedependent reversal of fibrosis, increased CV and suppressed spontaneous VF or cardiac arrest. The effects of RLX on cardiac fibrosis and CV were previously reported in hearts from aged [13] and chronically hypertensive rats. [14]

Optical mapping of PAH hearts revealed two dominant phenotypes as described above, but most intriguing was the robust rescue of both phenotypes by DTT which implied that these hearts had a compromised glutathione metabolism. Glutathione dysregulation has been implicated in the etiology and progression of a wide range of human diseases including heart failure.[210] There is mounting evidence of energy deficiency in heart failure associated with a disruption of oxidative phosphorylation, [211] and an augmentation of reactive oxygen species which readily oxidize free thiols.[212] In turn, the oxidation-reduction of reactive thiols on Ryanodine receptors were shown to reversibly open-close the channel [213] and was shown to become oxidized in ischemic [214] and failing hearts.[215] A lower ratio of reduced to oxidized glutathione (GSH/GSSG) or a the loss of cytosolic GSH leads to abnormal $\mathrm{Ca}^{2+}$ handling, and $\mathrm{Ca}^{2+}$ alternans in a canine model of sudden cardiac death.[216] As we showed for PAH hearts, restoring the redox status with exogenously added free thiols may be a promising therapy for HF. Likewise, RLX-treatment suppressed VF and cardiac arrest in PAH hearts most likely through a combination of mechanisms that may include the restoration of redox state. In further support of this hypothesis, preliminary RNAsequencing data in aged rats suggest a dysregulation of 
glutathione transferase mRNA expression which is reversed by relaxin treatment, and future studies should study these effects in detail.

Relaxin at the higher dose was highly cardioprotective through a reduction of cardiac fibrosis, an increase in AP CV and a marked suppression of VF and cardiac arrest. Doppler flow measurements of PAH hearts exhibited a pronounced secondary flow velocity peak indicative of significant pulmonary vascular remodeling which was eliminated by the high but not the low dose of RLX. The marked effect of high RLX dose on RV hemodynamics was aligned with a reduction of pulmonary peri-vascular fibrosis and a modest reduction in the number of partially occluded pulmonary arteries. The latter explain RLX's lack of changes in hypertrophy and systolic RV pressure. The study applied a high dose of RLX based on previous studies in spontaneously hypertensive and aged rats but the resulting serum concentration of RLX (15 $\mathrm{ng} / \mathrm{ml})$ did not reach the previous values $(75 \mathrm{ng} / \mathrm{ml})$ suggesting differences in biological activity of RLX in these models. It may well be that still higher doses might prove to be more beneficial particularly in terms of improving pulmonary vascular compliance. The study focused on male rats, yet considerable sex-differences in PAH patients and SuHx rats has been reported in regard to the number of plexiform lesions, with females exhibiting higher number of plexiform lesions than males.[33, 34] Future studies should examine sex-differences, higher concentrations of RLX and changes in cardiac thiol redox state in PAH hearts with and without RLX treatment. 


\subsection{Discussion}

Relaxin, a hormone discovered for its role in pregnancy, has since shown myriad beneficial effects in heart, liver and lung in multiple diseases including myocardial infarction, hypertension and aging. Despite these effects, however, there are still multiple unknowns on RLXs mechanisms of action. Studies have shown that, in renal fibroblasts, RLX blocks excess collagen expression from (myo)fibroblasts via interrupting TGF $\beta-S M A D$ signaling [43, 94] and involves nitric oxide-guanylyl cyclase signaling.[94] We show here that in addition to the TGF $\beta$ SMAD pathway, activation of Wnt signaling by RLX is also required to achieve suppression of fibrosis in adult primary cardiac fibroblasts. Further, there has been no work done to explain the mechanism of action of RLX to increase Nav1.5 to aid in arrhythmia suppression. We show that RLX activates canonical Wnt signaling in whole heart and in isolated cardiomyocytes, and that this activation is required for RLX to increase Nav1.5 expression. This action is at least partially achieved through increased Wnt1 and decreased DKK1 expression in LV and isolated cardiomyocytes. Notably, there is a lack of consensus on the effects of Wnt signaling and Nav1.5 regulation. Studies have shown that canonical Wnt signaling can suppress $[82,182]$ or increase [124] Nav1.5 expression, and appears to be chamber specific with gain or loss of Wnt signaling altering Nav1.5 or showing no change depending on the heart chamber studied.[217] While it is unclear what is causing these discrepancies, different cell types, rodent models, age or techniques to activate or inhibit Wnt signaling may begin to explain them. As described in section 1.4, 
various studies have shown that RLX can activate cAMP signaling in certain cell types, though it has not been determined if cAMP activation by RLX is necessary for Nav1.5 regulation. To explore this potential mechanism, isolated myocytes were treated with various adenylyl cyclase (AC), PKA and phosphodiesterase (PDE) inhibitors (not shown). The results were inconsistent and not reproducible with some inhibitors increasing or decreasing Nav1.5 directly and blocking RLX's effects, or multiple different AC, PKA or PDE inhibitors either having no effect or inconsistent effects between experiments. These results lowered our confidence that RLX was altering Nav1.5 expression through the cAMP pathway, and led us to find a different pathway interaction, namely: RLX-Wnt signaling.

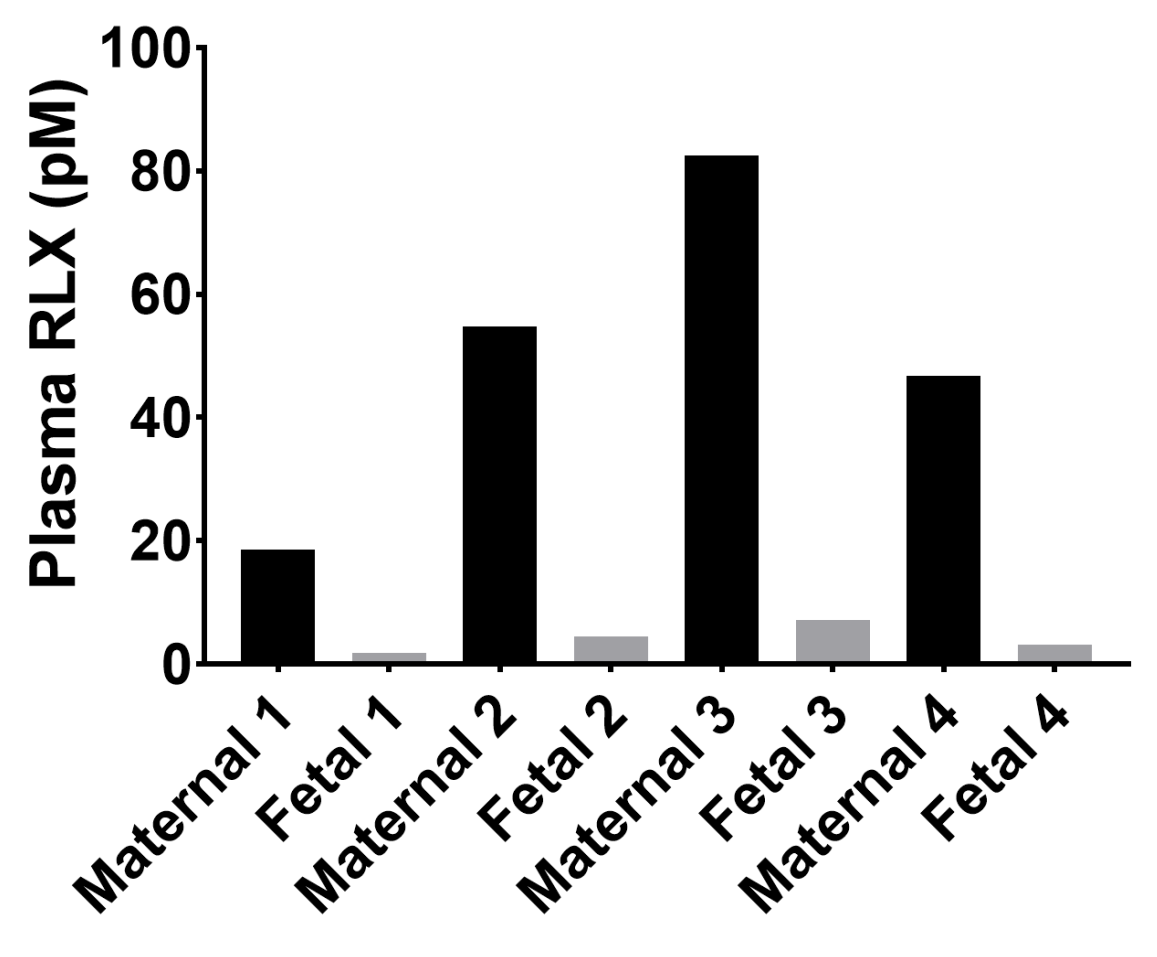

Figure 27. Relaxin Concentration in Maternal and Fetal Plasma. All maternal/fetal pairs displayed detectable levels of RLX in the plasma measured by ELISA. 
Our data on RLX-Wnt signaling become increasingly significant with the understanding that Wnt signaling is a major driver of cardio-genesis during development.[218, 219] Earlier studies had suggested that the developing fetus does not receive RLX from the mother's blood.[220] However, recent preliminary work in our lab suggests that, to the contrary, there are detectable levels of RLX in fetal plasma (Fig. 27).

Further, there is a strong $\left(\mathrm{R}^{2}=0.96, \mathrm{p}<0.05\right)$ correlation between $\mathrm{RLX}$ levels in the maternal plasma with that found in fetal plasma samples (Fig. 28), while no significant correlation exists between maternal or fetal RLX concentration and gestation length. While a limitation of this study is that we were only able to obtain maternal/fetal plasma samples relatively later in gestation ( $32-34$ weeks), this still provides compelling evidence that RLX may play a vital role in Wnt signaling regulation during development. This should be studied in detail.
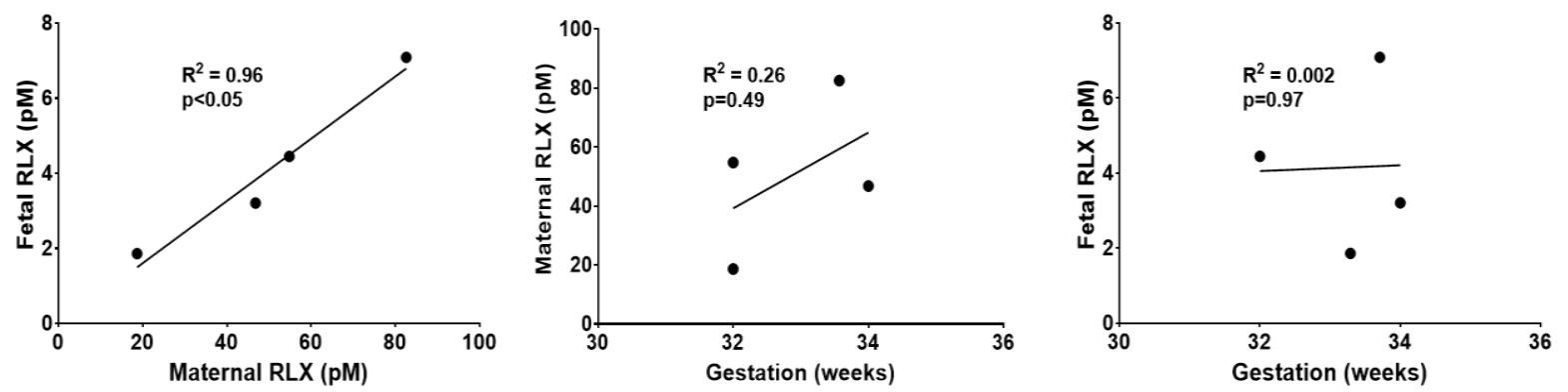

Figure 28. Relaxin Concentration Correlation Measurements in Maternal and Fetal Plasma. There was a strong positive correlation between fetal and maternal RLX plasma concentrations (left panel). There was no significant correlation between maternal (middle panel) or fetal (right panel) plasma concentration to gestation time.

Pulmonary arterial hypertension (PAH) is characterized by increased pulmonary artery pressure, pathological collagen deposition in the lung and occlusion of the pulmonary arterioles, for which there is no clinical cure. Increased pressure and pathological remodeling in PAH are thought to occur through an increased ratio of active vasoconstrictor molecules to 
vasodilators.[221-223] Based on a model of PAH induced by inhibition of the angiogenic growth factor, vascular endothelial growth factor (VEGF) in combination with hypoxia, which has been shown to induce PAH [223], we show that RLX can block the development of many of the pathological remodeling events that occur during PAH including: reduced RV fibrosis, increased action potential conduction velocity, reduced rates of arrhythmia development and inhibition of cardiac arrest. Further, RLX reduced plexiform lesion development and lowered the numbered of occluded pulmonary vessels. Interestingly, while it was shown that RLX can reduce cardiac arrest events, the mechanism is not clear. Using dithiothreitol (DTT), the hearts were rescued in a similar fashion. Since DTT is a sulfide reducing agent and had a similar effect as RLX in these experiments, we hypothesize that RLX may be acting as an anti-oxidant to restore energy imbalance that may occur in PAH. Preliminary data suggest that RLX alters the oxidative proteins Nrf2 and HO-1, though further work is needed in this regard. 


\subsection{Future Directions and Conclusions}

Preliminary work from our lab suggests that relaxin and Wnt signaling both mediate increased Nav1.5 through interplay with nitric oxide synthase (NOS). Interestingly, these effects do not appear to signal through the normal NOS-NO-sGC-cGMP pathway as inhibition of NOS, but not soluble guanylyl cyclase (sGC), blocked RLX and Wnt1 mediated increase in Nav1.5. While some work in cancer research [224] suggests NO can reduce DKK1 expression to activate Wnt signaling, there is no work in cardiac cells or tissue to confirm this, nor has this effect been compared in healthy and disease conditions. Further, we have studied only one ion channel protein, Nav1.5, in detail and it is unclear what effect relaxin-Wnt signaling interplay has on the various other ion channels relevant for the cardiac action potential and should be studied in detail in future work.

There are multiple avenues of study to pursue to understand RLX-Wnt mechanism of activation and if this effect is mediated by NO signaling. First, preliminary data from our lab suggests that RLX treatment of H9C2 cells leads to increased LRP6 phosphorylation, which could point to direct activation of canonical Wnt signaling through activation of the Wnt coreceptor by RLX. Second, evidence suggests that $\beta$-catenin bound to eNOS [225] or E-cadherin [226] can be disassociated with either of these two proteins and translocate to the nucleus to activate Wnt signaling. Interestingly, Mei et al [226] show that this separation of $\beta$-catenin from E-cadherin can be mediated via MMPs, proteins known to be regulated by RLX and NO [101, 
104]. Third, studies of RLX's anti-fibrotic effects in renal fibroblasts suggest that RXFP1 can form heterodimers with the angiotensin type 2 receptor (AT2R) to mediate its effects on collagen suppression. [227] This contrasted with no dimer formation between RXFP1 and the thyrotropinreleasing hormone, which was used as a control in this experiment, indicating some selectivity of interaction between RXFP1 and other receptors. Interestingly, they showed that while RXFP1AT2R dimerization was vital, RLX binding occurred only at RXFP1, with no interaction between RLX and AT2R directly. These data lead to intriguing ideas to study RXFP1-Fzd or RXFP1-LRP5/6 heterodimerization to directly activate canonical Wnt signaling to mediate RLX's effect on Nav1.5 expression. Fourth, while Chow et al [227] showed that RLX did not directly bind to the AT2R receptor, in studies involving the glucocorticoid receptor (GR), Dschietzig et al [105] showed that RLX binds directly to the GR to mediate its anti-inflammatory effects, and that this effect was conserved in the absence of the RLX receptor. These studies suggest complex binding and signaling mechanisms of RLX and its receptor to other signaling pathways, which leads to the hypothesis that RLX/RXFP1 may be interacting directly with Fzd/LRP5/6 to activate canonical Wnt signaling to increase Nav1.5 expression. Further preliminary evidence from our lab strengthening this position, suggests that in contrast to data in fibroblasts which show a conserved anti-fibrotic effect of a RLX mimetic (B7-33) with the RLX A-chain removed [116], there is no effect on Nav1.5 expression in isolated cardiomyocytes by B7-33. This suggests that both chains are necessary to mediate all RLX's effects, and that the Achain may be vital for RLX/RXFP1 coupling to non-RXFP1 receptors, or to activate some as yet unexplored signaling mechanism.

While we have shown that Wnt signaling is necessary for RLX's actions on Nav1.5 and RLX's anti-fibrotic effects in aged myocytes and cardiac fibroblasts, respectively, the precise 
signaling mechanisms are not clear. Data from our lab (not shown) suggest that at 48-hours of RLX treatment of fibroblasts, in contrast to the data seen in cardiomyocytes, there is no accumulation of $\beta$-catenin in the nucleus in cardiac fibroblasts. It would be useful therefore, to do a time-course study to determine if $\beta$-catenin accumulation in the fibroblast and myocyte nucleus is time or concentration dependent and how this differs between cell type.

Similarly, studies of Wnt-TGF $\beta$ signaling interactions would be useful to determine if RLX mediates suppression of TGF $\beta$ signaling in a Wnt dependent manner. Further, similar studies as discussed in myocytes above should be performed to determine if RLX acts directly on Fzd or LRP5/6 to activate Wnt signaling or is acting downstream, and to determine if the signaling mechanism is conserved between cell type.

Finally, and perhaps most intriguingly, preliminary ELISA data from our lab suggests that fetal blood receives RLX from maternal blood (Fig. 27 and Fig. 28). Given that Wnt signaling is highly active during development and is quiescent after birth, studies exploring RLX's effects on both canonical and non-canonical Wnt signaling in embryonic and fetal tissue could provide breakthrough understanding on cardiovascular development during gestation and if RLX is a master regulator of these events during pregnancy.

In conclusion, RLX has been shown to significantly improve cardiac action potential conduction through increased Nav1.5 expression, correction of cell-cell junctional protein mislocalization and expression and reduce fibrosis. These beneficial effects of RLX appear to be mediated through a complex interplay between RLX-Wnt-TGFß-NO signaling, which is largely unknown. Future work should include elucidation of the mechanisms of this interplay necessary for RLX's effects so that effective mimetics can be produced which replicate the effects of RLX seen in ion channel and collagen expression modulation. 


\section{Bibliography}

1. Bathgate, R.A., Halls, M.L., van der Westhuizen, E.T., Callander, G.E., Kocan, M. and Summers, R.J., 2013. Relaxin family peptides and their receptors, Physiol Rev. 93, 40580 .

2. Scott, D.J., Wilkinson, T., Tregear, G.W. and Bathgate, R.A.D., 2003. The relaxin peptide family and their novel G-protein coupled receptors, Letters in Peptide Science. 10, 393-400.

3. Hsu, S.Y., Nakabayashi, K., Nishi, S., Kumagai, J., Kudo, M., Sherwood, O.D. and Hsueh, A.J.W., 2002. Activation of Orphan Receptors by the Hormone Relaxin, Science. 295, 671-674.

4. Du, X.J., Bathgate, R.A., Samuel, C.S., Dart, A.M. and Summers, R.J., 2010. Cardiovascular effects of relaxin: from basic science to clinical therapy, Nat Rev Cardiol. 7, 48-58.

5. Sethi, A., Bruell, S., Patil, N., Hossain, M.A., Scott, D.J., Petrie, E.J., Bathgate, R.A. and Gooley, P.R., 2016. The complex binding mode of the peptide hormone $\mathrm{H} 2$ relaxin to its receptor RXFP1, Nat Commun. 7, 11344.

6. Dschietzig, T., Bartsch, C., Baumann, G. and Stangl, K., 2006. Relaxin-a pleiotropic hormone and its emerging role for experimental and clinical therapeutics, Pharmacol Ther. 112, 38-56.

7. Novak, J., Danielson, L.A., Kerchner, L.J., Sherwood, O.D., Ramirez, R.J., Moalli, P.A. and K.P. Conrad1, 2001. Relaxin is essential for renal vasodilation during pregnancy in conscious rats, J. Clin. Invest. 107, 1469-1475

8. Martin, B., Gabris-Weber, B.A., Reddy, R., Romero, G., Chattopadhyay, A. and Salama, G., 2018. Relaxin reverses inflammatory and immune signals in aged hearts, PLoS One. 13, e0190935.

9. Bani, D., 2008. Relaxin as a natural agent for vascular health, Vascular Health and Risk Management. 4, 515-524. 
10. Kohsaka, T., Min, G., Lukas, G., Trupin, S., Campbell, E.T. and Sherwood, O.D., 1998. Identification of Specific Relaxin-Binding Cells in the Human Female, Biology of Reproduction. 59, 991-999

11. Lekgabe, E.D., Kiriazis, H., Zhao, C., Xu, Q., Moore, X.L., Su, Y., Bathgate, R.A., Du, X.J. and Samuel, C.S., 2005. Relaxin reverses cardiac and renal fibrosis in spontaneously hypertensive rats, Hypertension. 46, 412-8.

12. E J Williams, R C Benyon, N Trim, R Hadwin, B H Grove, MJPArthur, E N Unemori and Iredale, J.P., 2001. Relaxin inhibits effective collagen deposition by cultured hepatic stellate cells and decreases rat liver fibrosis in vivo, Gut. 49, 577-583.

13. Henry, B.L., Gabris, B., Li, Q., Martin, B., Giannini, M., Parikh, A., Patel, D., Haney, J., Schwartzman, D.S., Shroff, S.G. and Salama, G., 2016. Relaxin suppresses atrial fibrillation in aged rats by reversing fibrosis and upregulating $\mathrm{Na}+$ channels, Heart Rhythm. 13, 983-91.

14. Parikh, A., Patel, D., McTiernan, C.F., Xiang, W., Haney, J., Yang, L., Lin, B., Kaplan, A.D., Bett, G.C., Rasmusson, R.L., Shroff, S.G., Schwartzman, D. and Salama, G., 2013. Relaxin suppresses atrial fibrillation by reversing fibrosis and myocyte hypertrophy and increasing conduction velocity and sodium current in spontaneously hypertensive rat hearts, Circ Res. 113, 313-21.

15. Alexiou, K., Wilbring, M., Matschke, K. and Dschietzig, T., 2013. Relaxin protects rat lungs from ischemia-reperfusion injury via inducible NO synthase: role of ERK-1/2, PI3K, and forkhead transcription factor FKHRL1, PLoS One. 8, e75592.

16. Dschietzig, T., Teichman, S., Unemori, E., Wood, S., Boehmer, J., Richter, C., Baumann, G. and Stangl, K., 2009. Intravenous recombinant human relaxin in compensated heart failure: a safety, tolerability, and pharmacodynamic trial, J Card Fail. $15,182-90$.

17. $\mathrm{Xu}, \mathrm{Y}$., Yu, Q. and Liu, Y., 2018. Serum relaxin-2 as a novel biomarker for prostate cancer, British Journal of Biomedical Science. 75, 145-148

18. Mazurek, J.A., Horne, B.D., Kelesidis, I., Salamon, J.N. and Zolty, R., 2013. Relaxin levels in pulmonary hypertension: a comparison between pulmonary arterial hypertension and diastolic heart failure-induced pulmonary hypertension, J Heart Lung Transplant. 32, 371-4.

19. Zhou, H., Qu, X., Gao, Z., Zheng, G., Lin, J., Su, L., Huang, Z., Li, H. and Huang, W., 2016. Relaxin Level in Patients With Atrial Fibrillation and Association with Heart Failure Occurrence: A STROBE Compliant Article, Medicine (Baltimore). 95, e3664.

20. Pintalhao, M., Castro-Chaves, P., Vasques-Novoa, F., Goncalves, F., Mendonca, L., FontesCarvalho, R., Lourenco, P., Almeida, P., Leite-Moreira, A. and Bettencourt, P., 
2016. Relaxin serum levels in acute heart failure are associated with pulmonary hypertension and right heart overload, Eur J Heart Fail.

21. Dschietzig, T., Richter, C., Bartsch, C., Laule, M., Armbruster, F.P., Baumann, G. and Stangl, K., 2001. The pregnancy hormone relaxin is a player in human heart failure, FASEB J. 15, 2187-95.

22. Rehfeldt, M., Sparwasser, A., Funk, E., Köhrle, J. and Bergmann, A., 2017. Quantification of Relaxin-2 Connecting Peptide (Pro-RLX2) in Human Blood Samples, JALM. 2, 322-334.

23. Debrah, D.O., Conrad, K.P., Danielson, L.A. and Shroff, S.G., 2005. Effects of relaxin on systemic arterial hemodynamics and mechanical properties in conscious rats: sex dependency and dose response, J Appl Physiol (1985). 98, 1013-20.

24. Debrah, D.O., Conrad, K.P., Jeyabalan, A., Danielson, L.A. and Shroff, S.G., 2005. Relaxin increases cardiac output and reduces systemic arterial load in hypertensive rats, Hypertension. 46, 745-50.

25. Debrah, D.O., Debrah, J.E., Haney, J.L., McGuane, J.T., Sacks, M.S., Conrad, K.P. and Shroff, S.G., 2011. Relaxin regulates vascular wall remodeling and passive mechanical properties in mice, J Appl Physiol (1985). 111, 260-71.

26. Van Linthout, S., Miteva, K. and Tschope, C., 2014. Crosstalk between fibroblasts and inflammatory cells, Cardiovasc Res. 102, 258-69.

27. Brown, T.R., Krogh-Madsen, T. and Christini, D.J., 2015. Computational Approaches to Understanding the Role of Fibroblast-Myocyte Interactions in Cardiac Arrhythmogenesis, Biomed Res Int. 2015, 465714.

28. Nguyen, T.P., Xie, Y., Garfinkel, A., Qu, Z. and Weiss, J.N., 2012. Arrhythmogenic consequences of myofibroblast-myocyte coupling, Cardiovasc Res. 93, 242-51.

29. Roell, W., Klein, A.M., Breitbach, M., Becker, T.S., Parikh, A., Lee, J., Zimmermann, K., Reining, S., Gabris, B., Ottersbach, A., Doran, R., Engelbrecht, B., Schiffer, M., Kimura, K., Freitag, P., Carls, E., Geisen, C., Duerr, G.D., Sasse, P., Welz, A., Pfeifer, A., Salama, G., Kotlikoff, M. and Fleischmann, B.K., 2018. Overexpression of Cx43 in cells of the myocardial scar: Correction of post-infarct arrhythmias through heterotypic cell-cell coupling, Sci Rep. 8, 7145.

30. Platonov, P.G., 2017. Atrial fibrosis: an obligatory component of arrhythmia mechanisms in atrial fibrillation?, J Geriatr Cardiol. 14, 233-237.

31. Lakatta, E.G., 2002. Age-associated Cardiovascular Changes in Health: Impact on Cardiovascular Disease in Older Persons, Heart Failure Reviews. 2, 29-49. 
32. Akhmetshina, A., Palumbo, K., Dees, C., Bergmann, C., Venalis, P., Zerr, P., Horn, A., Kireva, T., Beyer, C., Zwerina, J., Schneider, H., Sadowski, A., Riener, M.O., MacDougald, O.A., Distler, O., Schett, G. and Distler, J.H., 2012. Activation of canonical Wnt signalling is required for TGF-beta-mediated fibrosis, Nat Commun. 3, 735 .

33. Gordon, M.D. and Nusse, R., 2006. Wnt signaling: multiple pathways, multiple receptors, and multiple transcription factors, J Biol Chem. 281, 22429-33.

34. Dawson, K., Aflaki, M. and Nattel, S., 2013. Role of the Wnt-Frizzled system in cardiac pathophysiology: a rapidly developing, poorly understood area with enormous potential, J Physiol. 591, 1409-32.

35. He, W., Zhang, L., Ni, A., Zhang, Z., Mirotsou, M., Mao, L., Pratt, R.E. and Dzau, V.J., 2010. Exogenously administered secreted frizzled related protein 2 (Sfrp2) reduces fibrosis and improves cardiac function in a rat model of myocardial infarction, Proc Natl Acad Sci U S A. 107, 21110-5.

36. Kobayashi, K., Luo, M., Zhang, Y., Wilkes, D.C., Ge, G., Grieskamp, T., Yamada, C., Liu, T.C., Huang, G., Basson, C.T., Kispert, A., Greenspan, D.S. and Sato, T.N., 2009. Secreted Frizzledrelated protein 2 is a procollagen $\mathrm{C}$ proteinase enhancer with a role in fibrosis associated with myocardial infarction, Nat Cell Biol. 11, 46-55.

37. Blyszczuk, P., Muller-Edenborn, B., Valenta, T., Osto, E., Stellato, M., Behnke, S., Glatz, K., Basler, K., Luscher, T.F., Distler, O., Eriksson, U. and Kania, G., 2017. Transforming growth factor-beta-dependent Wnt secretion controls myofibroblast formation and myocardial fibrosis progression in experimental autoimmune myocarditis, Eur Heart J. 38, 1413-1425.

38. Samuel, C.S., Cendrawan, S., Gao, X.M., Ming, Z., Zhao, C., Kiriazis, H., Xu, Q., Tregear, G.W., Bathgate, R.A. and Du, X.J., 2011. Relaxin remodels fibrotic healing following myocardial infarction, Lab Invest. 91, 675-90.

39. Sangaralingham, S.J., Huntley, B.K., Martin, F.L., McKie, P.M., Bellavia, D., Ichiki, T., Harders, G.E., Chen, H.H. and Burnett, J.C., Jr., 2011. The aging heart, myocardial fibrosis, and its relationship to circulating C-type natriuretic Peptide, Hypertension. 57, 201-7.

40. Wang, D., Luo, Y., Myakala, K., Orlicky, D.J., Dobrinskikh, E., Wang, X. and Levi, M., 2017. Serelaxin improves cardiac and renal function in DOCA-salt hypertensive rats, Sci Rep. 7, 9793.

41. Samuel, C.S., Hewitson, T.D., Zhang, Y. and Kelly, D.J., 2008. Relaxin ameliorates fibrosis in experimental diabetic cardiomyopathy, Endocrinology. 149, 3286-93. 
42. Du, X.-J., Samuel, C.S., Gao, X.-M., Zhao, L., Parry, L.J. and Tregear, G.W., 2003. Increased myocardial collagen and ventricular diastolic dysfunction in relaxin deficient mice: a genderspecific phenotype, Cardiovascular Research. 57, 395-405.

43. Samuel, C.S., Unemori, E.N., Mookerjee, I., Bathgate, R.A., Layfield, S.L., Mak, J., Tregear, G.W. and Du, X.J., 2004. Relaxin modulates cardiac fibroblast proliferation, differentiation, and collagen production and reverses cardiac fibrosis in vivo, Endocrinology. 145, 4125-33.

44. Unemori, E.N. and Amento, E.P., 1990. Relaxin Modulates Synthesis and Secretion of Procollagenase and Collagen by Human Dermal Fibroblasts*, The Journal of Biological Chemistry. 265, 10681-10685.

45. Palejwala, S., Stein, D.E., Weiss, G., Monia, B.P., Tortoriello, D. and Goldsmith, L.T., 2001. Relaxin Positively Regulates Matrix Metalloproteinase Expression in Human Lower Uterine Segment Fibroblasts Using a Tyrosine Kinase Signaling Pathway, Endocrinology. 142, 3405-3413.

46. Palejwala, S., Stein, D., Wojtczuk, A., Weiss, G. and Goldsmith, L.T., 1998. Demonstration of a Relaxin Receptor and Relaxin Stimulated Tyrosine Phosphorylation in Human Lower Uterine Segment Fibroblasts*, Endocrinology. 139, 1208-1212.

47. Heeg, M.H., Koziolek, M.J., Vasko, R., Schaefer, L., Sharma, K., Muller, G.A. and Strutz, F., 2005. The antifibrotic effects of relaxin in human renal fibroblasts are mediated in part by inhibition of the Smad2 pathway, Kidney Int. 68, 96-109.

48. Mookerjee, I., Hewitson, T.D., Halls, M.L., Summers, R.J., Mathai, M.L., Bathgate, R.A., Tregear, G.W. and Samuel, C.S., 2009. Relaxin inhibits renal myofibroblast differentiation via RXFP1, the nitric oxide pathway, and Smad2, FASEB J. 23, 1219-29.

49. Sassoli, C., Chellini, F., Pini, A., Tani, A., Nistri, S., Nosi, D., Zecchi-Orlandini, S., Bani, D. and Formigli, L., 2013. Relaxin prevents cardiac fibroblast-myofibroblast transition via notch-1mediated inhibition of TGF-beta/Smad3 signaling, PLoS One. 8, e63896.

50. Zhou, X., Chen, X., Cai, J.J., Chen, L.Z., Gong, Y.S., Wang, L.X., Gao, Z., Zhang, H.Q., Huang, W.J. and Zhou, H., 2015. Relaxin inhibits cardiac fibrosis and endothelialmesenchymal transition via the Notch pathway, Drug Des Devel Ther. 9, 4599-611.

51. Squecco, R., Sassoli, C., Garella, R., Chellini, F., Idrizaj, E., Nistri, S., Formigli, L., Bani, D. and Francini, F., 2015. Inhibitory effects of relaxin on cardiac fibroblast-tomyofibroblast transition: an electrophysiological study, Exp Physiol. 100, 652-66.

52. Chilton, L., Giles, W.R. and Smith, G.L., 2007. Evidence of intercellular coupling between cocultured adult rabbit ventricular myocytes and myofibroblasts, J Physiol. 583, 225-36. 
53. Sridhar, S., Vandersickel, N. and Panfilov, A.V., 2017. Effect of myocyte-fibroblast coupling on the onset of pathological dynamics in a model of ventricular tissue, Sci Rep. 7,40985 .

54. Vasquez, C. and Morley, G.E., 2012. The origin and arrhythmogenic potential of fibroblasts in cardiac disease, J Cardiovasc Transl Res. 5, 760-7.

55. Askar, S.F.A., Ramkisoensing, A.A., Schalij, M.J., Bingen, B.O., Swildens, J., van der Laarse, A., Atsma, D.E., de Vries, A.A.F., Ypey, D.L. and Pijnappels, D.A., 2011. Antiproliferative treatment of myofibroblasts prevents arrhythmias in vitro by limiting myofibroblast-induced depolarization, Cardiovascular Research. 90, 295-304.

56. Li, G.R., Sun, H.Y., Chen, J.B., Zhou, Y., Tse, H.F. and Lau, C.P., 2009. Characterization of multiple ion channels in cultured human cardiac fibroblasts, PLoS One. 4, e7307.

57. Chatelier, A., Mercier, A., Tremblier, B., Theriault, O., Moubarak, M., Benamer, N., Corbi, P., Bois, P., Chahine, M. and Faivre, J.F., 2012. A distinct de novo expression of Nav1.5 sodium channels in human atrial fibroblasts differentiated into myofibroblasts, $\mathrm{J}$ Physiol. 590, 4307-19.

58. Koivumaki, J.T., Clark, R.B., Belke, D., Kondo, C., Fedak, P.W., Maleckar, M.M. and Giles, W.R., 2014. $\mathrm{Na}(+)$ current expression in human atrial myofibroblasts: identity and functional roles, Front Physiol. 5, 275.

59. Yuan, Y., Zhang, Y., Han, X., Li, Y., Zhao, X., Sheng, L. and Li, Y., 2017. Relaxin alleviates TGFbeta1-induced cardiac fibrosis via inhibition of Stat3-dependent autophagy, Biochem Biophys Res Commun. 493, 1601-1607.

60. Wang, P., Li, M., Dong, L., Chen, H., Su, W. and Wang, Y.P., 2017. Relaxin Inhibits Cardiac Fibrosis in Diabetic Rats: Roles of Protein Kinase Cdelta, Exp Clin Endocrinol Diabetes.

61. Unemori, E.N., Pickford, L.B., Salles, A.L., Piercy, C.E., Grove, B.H., Erikson, M.E. and Amento, E.P., 1996. Relaxin induces an extracellular matrix-degrading phenotype in human lung fibroblasts in vitro and inhibits lung fibrosis in a murine model in vivo, $\mathbf{J}$ Clin Invest. 98, 273945.

62. Wakili, R., Voigt, N., Kaab, S., Dobrev, D. and Nattel, S., 2011. Recent advances in the molecular pathophysiology of atrial fibrillation, J Clin Invest. 121, 2955-68.

63. Qu, Z. and Weiss, J.N., 2015. Mechanisms of ventricular arrhythmias: from molecular fluctuations to electrical turbulence, Annu Rev Physiol. 77, 29-55. 
64. Hove-Madsen, L., Llach, A., Bayes-Genis, A., Roura, S., Rodriguez Font, E., Aris, A. and Cinca, J., 2004. Atrial fibrillation is associated with increased spontaneous calcium release from the sarcoplasmic reticulum in human atrial myocytes, Circulation. 110, 1358-63.

65. Liang, X., Xie, H., Zhu, P.H., Hu, J., Zhao, Q., Wang, C.S. and Yang, C., 2008. Ryanodine receptor-mediated $\mathrm{Ca} 2+$ events in atrial myocytes of patients with atrial fibrillation, Cardiology. 111, 102-10.

66. Vest, J.A., Wehrens, X.H., Reiken, S.R., Lehnart, S.E., Dobrev, D., Chandra, P., Danilo, P., Ravens, U., Rosen, M.R. and Marks, A.R., 2005. Defective cardiac ryanodine receptor regulation during atrial fibrillation, Circulation. 111, 2025-32.

67. Neef, S., Dybkova, N., Sossalla, S., Ort, K.R., Fluschnik, N., Neumann, K., Seipelt, R., Schondube, F.A., Hasenfuss, G. and Maier, L.S., 2010. CaMKII-dependent diastolic SR $\mathrm{Ca} 2+$ leak and elevated diastolic $\mathrm{Ca} 2+$ levels in right atrial myocardium of patients with atrial fibrillation, Circ Res. 106, 1134-44.

68. Chelu, M.G., Sarma, S., Sood, S., Wang, S., van Oort, R.J., Skapura, D.G., Li, N., Santonastasi, M., Müller, F.U., Schmitz, W., Schotten, U., Anderson, M.E., Valderrábano, M., Dobrev, D. and Wehrens, X.H.T., 2009. Calmodulin kinase IImediated sarcoplasmic reticulum $\mathrm{Ca} 2+$ leak promotes atrial fibrillation in mice, Journal of Clinical Investigation.

69. Bosch, R.F., Zeng, X., Grammer, J.B., Popovic, K., Mewis, C. and Kuhlkamp, V., 1999. Ionic mechanisms of electrical remodeling in human atrial fibrillation, Cardiovascular Research. 44, 121-131.

70. Yue, L., Feng, J., Gaspo, R., Li, G.-R., Wang, Z. and Nattel, S., 1997. Ionic Remodeling Underlying Action Potential Changes in a Canine Model of Atrial Fibrillation, Circulation Research. 81, 512-525.

71. Rizzo, S., Lodder, E.M., Verkerk, A.O., Wolswinkel, R., Beekman, L., Pilichou, K., Basso, C., Remme, C.A., Thiene, G. and Bezzina, C.R., 2012. Intercalated disc abnormalities, reduced $\mathrm{Na}(+)$ current density, and conduction slowing in desmoglein-2 mutant mice prior to cardiomyopathic changes, Cardiovasc Res. 95, 409-18.

72. Gaspo, R., Bosch, R.F., Bou-Abboud, E. and Nattel, S., 1997. Tachycardia-Induced Changes in Na+ Current in a Chronic Dog Model of Atrial Fibrillation, Circulation Research. 81, 1045-1052.

73. Han, X., Habuchi, Y. and Giles, W.R., 1994. Relaxin Increases Heart Rate by Modulating Calcium Current in Cardiac Pacemaker Cells, Circulation Research. 74, 537541. 
74. Piedras-Renteria, E.S., Sherwood, O.D. and Best, P.M., 1997. Effects of relaxin on rat atrial myocytes. I. Inhibition of Ito via PKA-dependent phosphorylation, American Physiological Society. 272, H1791-H1797.

75. Piedras-Renteria, E.S., Sherwood, O.D. and Best, P.M., 1997. Effects of relaxin on rat atrial myocytes. II. Increased calcium influx derived from action potential prolongation, American Journal of Physiology. 272, H1798-H1803.

76. Beiert, T., Knappe, V., Tiyerili, V., Stockigt, F., Effelsberg, V., Linhart, M., Steinmetz, M., Klein, S., Schierwagen, R., Trebicka, J., Roell, W., Nickenig, G., Schrickel, J.W. and Andrie, R.P., 2018. Chronic lower-dose relaxin administration protects from arrhythmia in experimental myocardial infarction due to anti-inflammatory and anti-fibrotic properties, Int J Cardiol. 250, 21-28.

77. Beiert, T., Tiyerili, V., Knappe, V., Effelsberg, V., Linhart, M., Stockigt, F., Klein, S., Schierwagen, R., Trebicka, J., Nickenig, G., Schrickel, J.W. and Andrie, R.P., 2017. Relaxin reduces susceptibility to post-infarct atrial fibrillation in mice due to anti-fibrotic and antiinflammatory properties, Biochem Biophys Res Commun.

78. Wang, D., Zhu, H., Yang, Q. and Sun, Y., 2016. Effects of relaxin on cardiac fibrosis, apoptosis, and tachyarrhythmia in rats with myocardial infarction, Biomed Pharmacother. 84, 348-355.

79. Bani, D., Masini, E., Bello, M.G., Bigazzi, M. and Sacchi, T.B., 1998. Relaxin protects against myocardial injury caused by ischemia and reperfusion in rat heart, American Journal ofPathology,. 52, 1367-1376.

80. Nistri, S., Cinci, L., Perna, A.M., Masini, E., Mastroianni, R. and Bani, D., 2008. Relaxin induces mast cell inhibition and reduces ventricular arrhythmias in a swine model of acute myocardial infarction, Pharmacol Res. 57, 43-8.

81. Ai, Z., Fischer, A., Spray, D.C., Brown, A.M.C. and Fishman, G.I., 2000. Wnt-1 regulation of connexin43 in cardiac myocytes, Journal of Clinical Investigation. 105, 161-171.

82. Liang, W., Cho, H.C. and Marban, E., 2015. Wnt signalling suppresses voltagedependent $\mathrm{Na}(+)$ channel expression in postnatal rat cardiomyocytes, J Physiol. 593, 1147-57.

83. Asimaki, A., Kapoor, S., Plovie, E., Karin Arndt, A., Adams, E., Liu, Z., James, C.A., Judge, D.P., Calkins, H., Churko, J., Wu, J.C., MacRae, C.A., Kléber, A.G., Saffitz, J.E., Kleber, A.G. and Saffitz, J.E., 2014. Identification of a new modulator of the intercalated disc in a zebrafish model of arrhythmogenic cardiomyopathy, Science Translational Medicine. 6, $240 \mathrm{ra74.}$ 
84. Chelko, S.P., Asimaki, A., Andersen, P., Bedja, D., Amat-Alarcon, N., DeMazumder, D., Jasti, R., MacRae, C.A., Leber, R., Kleber, A.G., Saffitz, J.E. and Judge, D.P., 2016. Central role for GSK3beta in the pathogenesis of arrhythmogenic cardiomyopathy, JCI Insight. 1.

85. Kuznetsova, L., Plesneva, S., Derjabina, N., Omeljaniuk, E. and Pertseva, M., 1999. On the mechanism of relaxin action: the involvement of adenylyl cyclase signalling system, Regulatory Peptides. 80, 33-39.

86. Bartsch, O., Bartlick, B. and Ivell, R., 2001. Relaxin signalling links tyrosine phosphorylation to phosphodiesterase and adenylyl cyclase activity, Molecular Human Reproduction. 7, 799-809.

87. Nguyen, B.T., Yang, L., Sanborn, B.M. and Dessauer, C.W., 2003. Phosphoinositide 3kinase activity is required for biphasic stimulation of cyclic adenosine 3',5'monophosphate by relaxin, Mol Endocrinol. 17, 1075-84.

88. Nguyen, B.T. and Dessauer, C.W., 2005. Relaxin stimulates protein kinase C zeta translocation: requirement for cyclic adenosine 3',5'-monophosphate production, Mol Endocrinol. 19, 1012-23.

89. Halls, M.L., Bathgate, R.A. and Summers, R.J., 2006. Relaxin family peptide receptors RXFP1 and RXFP2 modulate cAMP signaling by distinct mechanisms, Mol Pharmacol. 70, 214-26.

90. Halls, M.L. and Cooper, D.M., 2010. Sub-picomolar relaxin signalling by a preassembled RXFP1, AKAP79, AC2, beta-arrestin 2, PDE4D3 complex, EMBO J. 29, 2772-87.

91. Sarwar, M., Samuel, C.S., Bathgate, R.A., Stewart, D.R. and Summers, R.J., 2015. Serelaxin mediated signal transduction in human vascular cells: bell-shaped concentration-response curves reflect differential coupling to $\mathrm{G}$ proteins, Br J Pharmacol. 172, 1005-19.

92. Summers, R.J., Bathgate, R.A., Wade, J.D., van der Westhuizen, E.T. and Halls, M.L., 2009. Roles of the receptor, the ligand, and the cell in the signal transduction pathways utilized by the relaxin family peptide receptors 1-3, Ann N Y Acad Sci. 1160, 99-104.

93. Halls, M.L., Hewitson, T.D., Moore, X.L., Du, X.J., Bathgate, R.A. and Summers, R.J., 2009. Relaxin activates multiple cAMP signaling pathway profiles in different target cells, Ann N Y Acad Sci. 1160, 108-11.

94. Wang, C., Kemp-Harper, B.K., Kocan, M., Ang, S.Y., Hewitson, T.D. and Samuel, C.S., 2016. The Anti-fibrotic Actions of Relaxin Are Mediated Through a NO-sGC-cGMPDependent Pathway in Renal Myofibroblasts In Vitro and Enhanced by the NO Donor, Diethylamine NONOate, Front Pharmacol. 7, 91. 
95. Masini, E., Bani, D., Bello, M.G., Bigazzi, M., Mannaioni, P.F. and Sacchi, T.B., 1997. Relaxin Counteracts Myocardial Damage Induced by Ischemia-Reperfusion in Isolated Guinea Pig Hearts: Evidence for an Involvement of Nitric Oxide*, Endocrinology. 138, 4713-4720.

96. Masini, E., Bani, D., Bigazzi, M., Mannaioni, P.F. and Bani-Sacchi, T., 1994. Effects of relaxin on mast cells. In vitro and in vivo studies in rats and guinea pigs, J Clin Invest. 94, 1974-80.

97. Bani, D., Failli, P., Bello, M.G., Thiemermann, C., Sacchi, T.B., Bigazzi, M. and Masini, E., 1998. Relaxin Activates the L-Arginine-Nitric Oxide Pathway in Vascular Smooth Muscle Cells in Culture, Hypertension. 31, 1240-1247.

98. Quattrone, S., Chiappini, L., Scapagnini, G., Bigazzi, B. and Bani, D., 2004. Relaxin potentiates the expression of inducible nitric oxide synthase by endothelial cells from human umbilical vein in in vitro culture, Mol Hum Reprod. 10, 325-30.

99. Nistri, S., Chiappini, L., Sassoli, C. and Bani, D., 2003. Relaxin inhibits lipopolysaccharideinduced adhesion of neutrophils to coronary endothelial cells by a nitric oxide-mediated mechanism, FASEB J. 17, 2109-11.

100. Masini, E., Nistri, S., Vannacci, A., Bani Sacchi, T., Novelli, A. and Bani, D., 2004. Relaxin inhibits the activation of human neutrophils: involvement of the nitric oxide pathway, Endocrinology. 145, 1106-12.

101. Conrad, K.P. and Shroff, S.G., 2011. Effects of relaxin on arterial dilation, remodeling, and mechanical properties, Curr Hypertens Rep. 13, 409-20.

102. Dschietzig, T., 2003. Relaxin, a Pregnancy Hormone, Is a Functional Endothelin-1 Antagonist: Attenuation of Endothelin-1-Mediated Vasoconstriction by Stimulation of Endothelin Type-B Receptor Expression via ERK-1/2 and Nuclear Factor-kappaB, Circulation Research. 92, 32-40.

103. Kerchner, L.J., Novak, J., Hanley-Yanez, K., Doty, K.D., Danielson, L.A. and Conrad, K.P., 2005. Evidence against the hypothesis that endothelial endothelin B receptor expression is regulated by relaxin and pregnancy, Endocrinology. 146, 2791-7.

104. Chow, B.S., Chew, E.G., Zhao, C., Bathgate, R.A., Hewitson, T.D. and Samuel, C.S., 2012. Relaxin signals through a RXFP1-pERK-nNOS-NO-cGMP-dependent pathway to up-regulate matrix metalloproteinases: the additional involvement of iNOS, PLoS One. 7, e42714.

105. Dschietzig, T., Bartsch, C., Stangl, V., Baumann, G. and Stangl, K., 2004. Identification of the pregnancy hormone relaxin as glucocorticoid receptor agonist, The FASEB Journal. 
106. Dschietzig, T., Bartsch, C., Baumann, G. and Stangl, K., 2009. RXFP1-inactive relaxin activates human glucocorticoid receptor: further investigations into the relaxin-GR pathway, Regul Pept. 154, 77-84.

107. Raleigh, J.V., Mauro, A.G., Devarakonda, T., Marchetti, C., He, J., Kim, E., Filippone, S., Das, A., Toldo, S., Abbate, A. and Salloum, F.N., 2017. Reperfusion therapy with recombinant human relaxin-2 (Serelaxin) attenuates myocardial infarct size and NLRP3 inflammasome following ischemia/reperfusion injury via eNOS-dependent mechanism, Cardiovascular Research. 113, 609-619.

108. Ghosh, R.K., Banerjee, K., Tummala, R., Ball, S., Ravakhah, K. and Gupta, A., 2016. Serelaxin in Acute Heart Failure: Most Recent Update on Clinical and Preclinical Evidence, Cardiovasc Ther.

109. Teerlink, J.R., Metra, M., Felker, G.M., Ponikowski, P., Voors, A.A., Weatherley, B.D., Marmor, A., Katz, A., Grzybowski, J., Unemori, E., Teichman, S.L. and Cotter, G., 2009. Relaxin for the treatment of patients with acute heart failure (Pre-RELAX-AHF): a multicentre, randomised, placebo-controlled, parallel-group, dose-finding phase IIb study, The Lancet. 373, 1429-1439.

110. Teerlink, J.R., Cotter, G., Davison, B.A., Felker, G.M., Filippatos, G., Greenberg, B.H., Ponikowski, P., Unemori, E., Voors, A.A., Adams, K.F., Dorobantu, M.I., Grinfeld, L.R., Jondeau, G., Marmor, A., Masip, J., Pang, P.S., Werdan, K., Teichman, S.L., Trapani, A., Bush, C.A., Saini, R., Schumacher, C., Severin, T.M. and Metra, M., 2013. Serelaxin, recombinant human relaxin-2, for treatment of acute heart failure (RELAXAHF): a randomised, placebocontrolled trial, The Lancet. 381, 29-39.

111. Metra, M., Ponikowski, P., Cotter, G., Davison, B.A., Felker, G.M., Filippatos, G., Greenberg, B.H., Hua, T.A., Severin, T., Unemori, E., Voors, A.A. and Teerlink, J.R., 2013. Effects of serelaxin in subgroups of patients with acute heart failure: results from RELAX-AHF, Eur Heart J. 34, 3128-36.

112. Felker, G.M., Teerlink, J.R., Butler, J., Hernandez, A.F., Miller, A.B., Cotter, G., Davison, B.A., Filippatos, G., Greenberg, B.H., Ponikowski, P., Voors, A.A., Hua, T.A., Severin, T.M., Unemori, E. and Metra, M., 2014. Effect of serelaxin on mode of death in acute heart failure: results from the RELAX-AHF study, J Am Coll Cardiol. 64, 1591-8.

113. Filippatos, G., Teerlink, J.R., Farmakis, D., Cotter, G., Davison, B.A., Felker, G.M., Greenberg, B.H., Hua, T., Ponikowski, P., Severin, T., Unemori, E., Voors, A.A. and Metra, M., 2014. Serelaxin in acute heart failure patients with preserved left ventricular ejection fraction: results from the RELAX-AHF trial, Eur Heart J. 35, 1041-50.

114. McCullough, P.A., 2017. How Trialists and Pharmaceutical Sponsors Have Failed Us by Thinking That Acute Heart Failure Is a 48-Hour Illness, Am J Cardiol. 120, 505-508. 
115. Xiao, J., Huang, Z., Chen, C.Z., Agoulnik, I.U., Southall, N., Hu, X., Jones, R.E., Ferrer, M., Zheng, W., Agoulnik, A.I. and Marugan, J.J., 2013. Identification and optimization of smallmolecule agonists of the human relaxin hormone receptor RXFP1, Nat Commun. 4, 1953.

116. Hossain, M.A., Kocan, M., Yao, S.T., Royce, S.G., Nair, V.B., Siwek, C., Patil, N.A., Harrison, I.P., Rosengren, K.J., Selemidis, S., Summers, R.J., Wade, J.D., Bathgate, R.A.D. and Samuel, C.S., 2016. A single-chain derivative of the relaxin hormone is a functionally selective agonist of the $\mathrm{G}$ protein-coupled receptor, RXFP1, Chemical Science. 7, 3805-3819.

117. Marshall, S.A., O'Sullivan, K., Ng, H.H., Bathgate, R.A.D., Parry, L.J., Hossain, M.A. and Leo, C.H., 2017. B7-33 replicates the vasoprotective functions of human relaxin-2 (serelaxin), Eur J Pharmacol. 807, 190-197.

118. McBride, A., Hoy, A.M., Bamford, M.J., Mossakowska, D.E., Ruediger, M.P., Griggs, J., Desai, S., Simpson, K., Caballero-Hernandez, I., Iredale, J.P., Pell, T., Aucott, R.L., Holmes, D.S., Webster, S.P. and Fallowfield, J.A., 2017. In search of a small molecule agonist of the relaxin receptor RXFP1 for the treatment of liver fibrosis, Sci Rep. 7 , 10806.

119. Kocan, M., Sarwar, M., Ang, S.Y., Xiao, J., Marugan, J.J., Hossain, M.A., Wang, C., Hutchinson, D.S., Samuel, C.S., Agoulnik, A.I., Bathgate, R.A.D. and Summers, R.J., 2017. ML290 is a biased allosteric agonist at the relaxin receptor RXFP1, Sci Rep. 7, 2968.

120. Huang, Z., Myhr, C., Bathgate, R.A., Ho, B.A., Bueno, A., Hu, X., Xiao, J., Southall, N., Barnaeva, E., Agoulnik, I.U., Marugan, J.J., Ferrer, M. and Agoulnik, A.I., 2015. Activation of Relaxin Family Receptor 1 from Different Mammalian Species by Relaxin Peptide and SmallMolecule Agonist ML290, Front Endocrinol (Lausanne). 6, 128.

121. Kaftanovskaya, E.M., Soula, M., Myhr, C., Ho, B.A., Moore, S.N., Yoo, C., Cervantes, B., How, J., Marugan, J., Agoulnik, I.U. and Agoulnik, A.I., 2017. Human Relaxin Receptor Is Fully Functional in Humanized Mice and Is Activated by Small Molecule Agonist ML290, J Endocr Soc. 1, 712-725.

122. Kobalava, Z., Villevalde, S., Kotovskaya, Y., Hinrichsen, H., Petersen-Sylla, M., Zaehringer, A., Pang, Y., Rajman, I., Canadi, J., Dahlke, M., Lloyd, P. and Halabi, A., 2015. Pharmacokinetics of serelaxin in patients with hepatic impairment: a single-dose, open-label, parallel group study, Br J Clin Pharmacol. 79, 937-45.

123. Teerlink, J.R., Cotter, G., Davison, B.A., Felker, G.M., Filippatos, G., Greenberg, B.H., Ponikowski, P., Unemori, E., Voors, A.A., Adams, K.F., Jr., Dorobantu, M.I., Grinfeld, L.R., Jondeau, G., Marmor, A., Masip, J., Pang, P.S., Werdan, K., Teichman, S.L., Trapani, A., Bush, C.A., Saini, R., Schumacher, C., Severin, T.M., Metra, M. and Investigators, R.E.i.A.H.F., 2013. Serelaxin, recombinant human relaxin-2, for treatment 
of acute heart failure (RELAX-AHF): a randomised, placebo-controlled trial, Lancet. 381, 29-39.

124. Asimaki, A., et al., Arrhythmogenic Cardiomyopathy - New Insights into Disease Mechanisms and Drug Discovery. Prog Pediatr Cardiol, 2014. 37(1-2): p. 3-7.

125. D. P. Zipes et al., ACC/AHA/ESC 2006 guidelines for management of patients with ventricular arrhythmias and the prevention of sudden cardiac death: a report of the American College of Cardiology/American Heart Association Task Force and the European Society of Cardiology Committee for Practice Guidelines (Writing Committee to Develop Guidelines for Management of Patients With Ventricular Arrhythmias and the Prevention of Sudden Cardiac Death). Journal of the American College of Cardiology, Practice Guideline. 2006; 48, no. 5, e247-346.

126. G. Salama and S. M. Hwang, Simultaneous optical mapping of intracellular free calcium and action potentials from Langendorff perfused hearts. Current protocols in cytometry. 2009; Chapter 12, p. Unit 12.

127. Savio-Galimberti E, Darbar D. Atrial Fibrillation and SCN5A Variants. Card Electrophysiol Clin. 2014; 6(4):741-8.

128. Jessup MMD, Brozena SMD. Heart failure. New England Journal of Medicine. 2003; 348:2007-18.

129. Lakatta EG. Arterial and Cardiac Aging: Major Shareholders in Cardiovascular Disease Enterprises: Part I: Aging Arteries: A "Set Up" for Vascular Disease. Circulation. 2003; 107(1):139-46.

130. Braunwald E. Heart failure. JACC Heart Fail. 2013; 1(1):1-20.

131. Strait JB, Lakatta EG. Aging-associated cardiovascular changes and their relationship to heart failure. Heart Fail Clin. 2012; 8(1):143-64.

132. Hayashi H, Wang C, Miyauchi Y, Omichi C, Pak H, Zhou S, et al. Aging-Related Increase to Inducible Atrial Fibrillation in the Rat Model. Journal of Cardiovascular Electrophysiology. 2002; 13(8):801-8.

133. Licastro F, Candore G, Lio D, Porcellini E, Colonna-Romano G, Franceschi C, et al. Innate immunity and inflammation in ageing: a key for understanding age-related diseases. Immun Ageing. 2005; 2:8.

134. Markiewski MM, Lambris JD. The role of complement in inflammatory diseases from behind the scenes into the spotlight. Am J Pathol. 2007; 171(3):715-27.

135. Liu J, Wang H, Li J. Inflammation and Inflammatory Cells in Myocardial Infarction and Reperfusion Injury: A Double-Edged Sword. Clin Med Insights Cardiol. 2016; 10:79-84. 
136. Hofmann U, Frantz S. How can we cure a heart "in flame"? A translational view on inflammation in heart failure. Basic Res Cardiol. 2013; 108(4):356.

137. Kraakman MJ, Dragoljevic D, Kammoun HL, Murphy AJ. Is the risk of cardiovascular disease altered with anti-inflammatory therapies? Insights from rheumatoid arthritis. Clin Transl Immunology. 2016; 5 (5):e84.

138. Bacchiega BC, Bacchiega AB, Usnayo MJ, Bedirian R, Singh G, Pinheiro GD. Interleukin 6 Inhibition and Coronary Artery Disease in a High-Risk Population: A Prospective Community-Based Clinical Study. J Am Heart Assoc. 2017; 6(3).

139. Anker SD, von Haehling S. Inflammatory mediators in chronic heart failure: an overview. Heart. 2004; 90(4):464-70.

140. Lind L, Sundstrom J, Stenemo M, Hagstrom E, Arnlov J. Discovery of new biomarkers for atrial fibrillation using a custom-made proteomics chip. Heart. 2016. Braunwald E. Biomarkers in Heart Failure. NEJM. 2008; 358:2148-59.

141. Braunwald E. Heart failure. JACC Heart Fail. 2013; 1(1):1-20.

142. Gaggin HK, Januzzi JL Jr. Biomarkers and diagnostics in heart failure. Biochim Biophys Acta. 2013; 1832(12):2442-50.

143. Chan MM, Santhanakrishnan R, Chong JP, Chen Z, Tai BC, Liew OW, et al. Growth differentiation factor 15 in heart failure with preserved vs. reduced ejection fraction. Eur J Heart Fail. 2016; 18(1):81-8.

144. Brandt RR, Wright RS, Redfield MM, Burnett JC. Atrial natriuretic peptide in heart failure. Journal of the American College of Cardiology. 1993; 22(4):A86-A92.

145. Wallentin L, Hijazi Z, Andersson U, Alexander JH, De Caterina R, Hanna M, et al. Growth differentiation factor 15, a marker of oxidative stress and inflammation, for risk assessment in patients with atrial fibrillation: insights from the Apixaban for Reduction in Stroke and Other Thromboembolic Events in Atrial Fibrillation (ARISTOTLE) trial. Circulation. 2014; 130(21):1847-58.

146. Parini Paolo, Angelin Bo, Cholesterol Rudling M. and Lipoprotein Metabolism in Aging Reversal of Hypercholesterolemia by Growth Hormone Treatment in Old Rats. Arterioscler Thromb Vasc Biol. 1999; 19:832-9.

147. Lakatta EG. So! What's aging? Is cardiovascular aging a disease? J Mol Cell Cardiol. 2015; 83:1-13.

148. de Magalhaes JP, Curado J, Church GM. Meta-analysis of age-related gene expression profiles identifies common signatures of aging. Bioinformatics. 2009; 25(7):875-81. 
149. Zahn JM, Poosala S, Owen AB, Ingram DK, Lustig A, Carter A, et al. AGEMAP: A Gene Expression Database for Aging in Mice. PLOS Genetics. 2007; 3(11):e201.

150. Yang Jialiang, Huang Tao, Petralia Francesca, Long Quan, Zhang Bin, Argmann Carmen, et al. Synchronized age-related gene expression changes across multiple tissues in human and the link to complex diseases. Nature. 2015;5.

151. Boluyt Marvin O, Converso Kimber, Hwang Hyun Seok, Mikkor Agdas, Russell MW2. Echocardiographic assessment of age-associated changes in systolic and diastolic function of the female F344 rat heart. J Appl Physiol. 2004; 96:822-8.

152. Sangaralingham SJ, Huntley BK, Martin FL, McKie PM, Bellavia D, Ichiki T, et al. The aging heart, myocardial fibrosis, and its relationship to circulating C-type natriuretic Peptide. Hypertension. 2011; 57 (2):201-7.

153. Teichman SL, Unemori E, Teerlink JR, Cotter G, Metra M. Relaxin: review of biology and potential role in treating heart failure. Curr Heart Fail Rep. 2010; 7(2):75-82..

15.4 Franceschi C, Campisi J. Chronic inflammation (inflammaging) and its potential contribution to ageassociated diseases. J Gerontol A Biol Sci Med Sci. 2014; 69 Suppl 1:S4-9.

155. Lund SA, Giachelli CM, Scatena M. The role of osteopontin in inflammatory processes. J Cell Commun Signal. 2009; 3(3-4):311-22.

156. Huerta A, Lopez B, Ravassa S, San Jose G, Querejeta R, Beloqui O, et al. Association of cystatin $\mathrm{C}$ with heart failure with preserved ejection fraction in elderly hypertensive patients: potential role of altered collagen metabolism. J Hypertens. 2016; 34(1):130-8.

157. Martinez Fernando $\mathrm{O}$, Gordon $\mathrm{S}$. The M1 and M2 paradigm of macrophage activation: time for reassessment. F1000 Prime Reports. 2014; 6(13).

158. Schmied M, Breitschopf H, Gold R, Zischler H, Rothe G, Wekerle H, et al. Apoptosis of T Lymphocytes in Experimental Autoimmune Encephalomyelitis.pdf>. american Journal of Pathology. 1993; 143(2).

159. Lenardo M, Chan FK-M, Hornung F, McFarland H, Siegel R, Wang J, et al. MATURE T LYMPHOCYTE APOPTOSIS - Immune Regulation in a Dynamic and Unpredictable Antigenic Environment. Annu Rev Immunology. 1999; 17:221-53.

160. Hotchkiss RS, Tinsley KW, Swanson PE, Chang KC, Cobb JP, Buchman TG, et al. Prevention of lymphocyte cell death in sepsis improves survival in mice. PNAS. 1999; 96(25):14541-6. 
161. Thaker Youg R, Rudd C. Distinct NF-kB activation pathways engaged by T-cell receptor and co-receptor CD28 on T-cells. Inflammation and Cell Signaling. 2015; 2:e613.

162. Freeley M, Long A. Regulating the Regulator: Phosphorylation of PKC theta in T Cells. Front Immunol. 2012; 3:227.

163. Lo“hning M, Hutloff A, Kallinich T, Mages HW, Bonhagen K, Radbruch A, et al. Expression of ICOS In Vivo Defines CD4+Effector T Cells with High Inflammatory Potential and a Strong Bias for Secretion of Interleukin 10. The Journal of Experimental Medicine. 2003; 197(2):181-93.

164. Fric J, Zelante T, Wong AY, Mertes A, Yu HB, Ricciardi-Castagnoli P. NFAT control of innate immunity. Blood. 2012; 120(7):1380-9.

165. Berger A. Science commentary: Th1 and Th2 responses: what are they? BMJ. 2000; 321:424.

166. Lin Q, Fang D, Fang J, Ren X, Yang X, Wen F, et al. Impaired wound healing with defective expression of chemokines and recruitment of myeloid cells in TLR3-deficient mice. J Immunol. 2011; 186(6):3710- 7.

167. Klein SL, Flanagan KL. Sex differences in immune responses. Nat Rev Immunol. 2016; 16(10):626-38.

168. Duncan Bruce B, Schmidt Maria Ine^s, Pankow James S, Couper David, Vigo Alvaro, Hoogeveen Ron, et al. Low-Grade Systemic Inflammation and the Development of Type 2 Diabetes: The Atherosclerosis Risk in Communities Study. Diabetes. 52:1799-805.

169. Hohensinner PJ, Niessner A, Huber K, Weyand CM, Wojta J. Inflammation and cardiac outcome. Curr Opin Infect Dis. 2011; 24(3):259-64.

170. Platonov PG, Mitrofanova LB, Orshanskaya V, Ho SY. Structural abnormalities in atrial walls are associated with presence and persistency of atrial fibrillation but not with age. $\mathrm{J}$ Am Coll Cardiol. 2011; 58 (21):2225-32.

171. Soneson C, Love MI, Robinson MD. Differential analyses for RNA-seq: transcript-level estimates improve gene-level inferences. F1000Res. 2015; 4:1521.

172. Consortium TG. The Genotype-Tissue Expression (GTEx) pilot analysis: Multitissue gene regulation in humans. Science. 2015; 346(6235):648-60.

173. Muehlschlegel Jochen D, Christodoulou Danos C, McKean David, Gorham Joshua, Mazaika Erica, Heydarpour Mahyar, et al. Using Next-generation RNA Sequencing to Examine Ischemic Changes Induced by Cold Blood Cardioplegia on the Human Left Ventricular Myocardium Transcriptome. Anesthesiology. 2015; 122:537-50. 
174. Himes BE, Jiang X, Wagner P, Hu R, Wang Q, Klanderman B, et al. RNA-Seq transcriptome profiling identifies CRISPLD2 as a glucocorticoid responsive gene that modulates cytokine function in airway smooth muscle cells. PLoS One. 2014; 9(6):e99625.

175. Medzhitov R, Horng T. Transcriptional Control of the Inflammatory Response. Nature. 2009; 9:692-703.

176. Lakatta, E. G. \& Levy, D. Arterial and cardiac aging: major shareholders in cardiovascular disease enterp.rises: Part II: the aging heart in health: links to heart disease. Circulation. 2003; 107, 346-354.

177. Strait, J. B. \& Lakatta, E. G. Aging-associated cardiovascular changes and their relationship to heart failure. Heart Fail Clin. 2012; 8, 143-164.

178. Dun, W. \& Boyden, P. A. Aged atria: electrical remodeling conducive to atrial fibrillation. J Interv Card Electrophysiol. 2009; 25, 9-18.

179. Dawson, K., Aflaki, M. \& Nattel, S. Role of the Wnt-Frizzled system in cardiac pathophysiology: a rapidly developing, poorly understood area with enormous potential. J Physiol. 2013; 591, 1409-1432.

180. Paige, S. L. et al. Endogenous Wnt/beta-catenin signaling is required for cardiac differentiation in human embryonic stem cells. PloS one. 2010; 5, e11134.

181. Mehta, A. et al. Phasic modulation of Wnt signaling enhances cardiac differentiation in human pluripotent stem cells by recapitulating developmental ontogeny. Biochim Biophys Acta. 2014; 1843, 2394-2402.

182. Wang, N. et al. Activation of Wnt/beta-catenin signaling by hydrogen peroxide transcriptionally inhibits NaV1.5 expression. Free Radic Biol Med. 2016; 96, 34-44.

183. Asimaki, A., Kleber, A. G., MacRae, C. A. \& Saffitz, J. E. Arrhythmogenic Cardiomyopathy - New Insights into Disease Mechanisms and Drug Discovery. Prog Pediatr Cardiol. 2014; 37, 3-7.

184. Thompson, V. C. et al. Relaxin drives Wnt signaling through upregulation of PCDHY in prostate cancer. Prostate. 2010; 70, 1134-1145.

185. Neschadim, A., Summerlee, A. J. \& Silvertown, J. D. Targeting the relaxin hormonal pathway in prostate cancer. Int J Cancer. 2015; 137, 2287-2295.

186. Liu, S. et al. Inappropriate activation of androgen receptor by relaxin via beta-catenin pathway19. Oncogene. 2008; 27, 499-505. 
187. King, J. H., Huang, C. L. \& Fraser, J. A. Determinants of myocardial conduction velocity: implications for arrhythmogenesis. Front Physiol. 2013; 4, 154.

188. Sakane, H., Yamamoto, H. \& Kikuchi, A. LRP6 is internalized by Dkk1 to suppress its phosphorylation in the lipid raft and is recycled for reuse. J Cell Sci. 2010; 123, 360-368.

189. N. C. Bloodworth, J. D. West, and W. D. Merryman. Microvessel mechanobiology in pulmonary arterial hypertension: cause and effect. Hypertension. 2015; 65, 483-9.

190. Y. C. Lai, K. C. Potoka, H. C. Champion, A. L. Mora, and M. T. Gladwin. Pulmonary arterial hypertension: the clinical syndrome. Circulation research. 2014; 115, 115-30.

191. G. Simonneau et al., Updated clinical classification of pulmonary hypertension. Journal of the American College of Cardiology, Review. 2009; 54, no. 1 Suppl, pp. S43-54.

192. A. Rajdev, H. Garan, and A. Biviano. Arrhythmias in pulmonary arterial hypertension. Progress in cardiovascular diseases, Review. 2012; 55, 180-6.

193. K. R. Kumar, S. N. Mandleywala, and M. S. Link. Atrial and Ventricular Arrhythmias in Hypertrophic Cardiomyopathy," Cardiac electrophysiology clinics, Review. 2015; 7, 173-186.

194. S. Kumar, W. G. Stevenson, and R. M. John. Arrhythmias in Dilated Cardiomyopathy. Cardiac electrophysiology clinics, Review. 2015; 7, 221-233.

195. M. Shenasa, H. Shenasa, and N. El-Sherif. Left Ventricular Hypertrophy and Arrhythmogenesis. Cardiac electrophysiology clinics, Review. 2015; 7, 207-220.

196. M. C. Honigberg and M. M. Givertz. Arrhythmias in Peripartum Cardiomyopathy," (in Eng), Cardiac electrophysiology clinics, Review. 2015; 7, 309-317.

197. C. Y. Miyake and J. J. Kim. Arrhythmias in Left Ventricular Noncompaction. Cardiac electrophysiology clinics, Review. 2015; 7, 319-330.

198. A. F. Folino et al., Ventricular arrhythmias and autonomic profile in patients with primary pulmonary hypertension. Lung, Comparative Study. 2003; 181, 321-8.

199. Z. Hong-liang et al., Heart rate-corrected QT interval and QT dispersion in patients with pulmonary hypertension. Wiener klinische Wochenschrift. 2009; 121, 330-3.

200. K. Abe et al., Formation of plexiform lesions in experimental severe pulmonary arterial hypertension. Circulation. 2010; 121, 2747-54.

201. O. Rafikova et al., Bosentan inhibits oxidative and nitrosative stress and rescues occlusive pulmonary hypertension. Free Radic Biol Med. 2013; 56, 28-43. 
202. D. Bani, S. K. Yue, and M. Bigazzi. Clinical profile of relaxin, a possible new drug for human use. Current drug safety, Review. 2009; 4, 238-49.

203. C. A. Tozzi et al., Recombinant human relaxin reduces hypoxic pulmonary hypertension in the rat. Pulm Pharmacol Ther. 2005; 18, 346-53.

204. C. S. Samuel et al., Relaxin deficiency in mice is associated with an age-related progression of pulmonary fibrosis. FASEB J. 2003; 17, 121-3.

205. T. Dschietzig et al., Intravenous recombinant human relaxin in compensated heart failure: a safety, tolerability, and pharmacodynamic trial. Journal of cardiac failure. 2009; 15, 182-90.

206. S. L. Teichman et al., Relaxin, a pleiotropic vasodilator for the treatment of heart failure. Heart failure reviews, Review. 2009; 14, 321-9.

207. E. Stacher et al., Modern age pathology of pulmonary arterial hypertension. Am J Respir Crit Care Med, 2012; 186, 261-72.

208. S. P. Tofovic, V. P. Bilan, E. K. Jackson, and F. Schneider. Sugen 5416 Dose-HypoxiaNormoxia-Gender Interaction In Angioproliferative Pulmonary Hypertension In Rats. Am J Respir Crit Care Med. 2014; 189, A5566.

209. S. P. Tofovic, V. P. Bilan, E. K. Jackson, and F. Schneider. Sugene 5416 Dose-HypoxiaNormoxia-Gender Interaction In Angioproliferative Pulmonary Hypertension In Rats. American Journal of Respiratory and Critical Care Medicine. 2014; 189, A5566.

210. N. Ballatori, S. M. Krance, S. Notenboom, S. Shi, K. Tieu, and C. L. Hammond. Glutathione dysregulation and the etiology and progression of human diseases. Biol Chem. 2009; 390, 191-214.

211. F. L. Sheeran and S. Pepe. Energy deficiency in the failing heart: linking increased reactive oxygen species and disruption of oxidative phosphorylation rate. Biochim Biophys Acta. 2006; 1757, 543-52.

212. A. M. Koning et al., Serum free thiols in chronic heart failure. Pharmacol Res. 2016; 111, 452-458.

213. N. F. Zaidi, C. F. Lagenaur, J. J. Abramson, I. Pessah, and G. Salama. Reactive disulfides trigger $\mathrm{Ca} 2+$ release from sarcoplasmic reticulum via an oxidation reaction. J Biol Chem. $1989 ; 264,21725-36$.

214. E. V. Menshikova and G. Salama. Cardiac ischemia oxidizes regulatory thiols on ryanodine receptors: captopril acts as a reducing agent to improve $\mathrm{Ca} 2+$ uptake by ischemic sarcoplasmic reticulum. J Cardiovasc Pharmacol. 2000; 36, 656-68. 
215. D. Terentyev et al., Redox modification of ryanodine receptors contributes to sarcoplasmic reticulum Ca2+ leak in chronic heart failure. Circ Res. 2008; 103, 1466-72.

216. A. E. Belevych et al., Redox modification of ryanodine receptors underlies calcium alternans in a canine model of sudden cardiac death. Cardiovasc Res. 2009; 84, 387-95.

217. G. Li., et al., Differential Wnt-mediated programming and arrhythmogenesis in right versus left ventricles. J Mol Cell Cardiol, 2018. 123, 92-107.

218. G. Ozhan, and G. Weidinger., Wnt/beta-catenin signaling in heart regeneration. Cell Regen (Lond), 2015. 4, 3.

219. S. Gessert, and M. Kuhl., The multiple phases and faces of wnt signaling during cardiac differentiation and development. Circ Res, 2010. 107, 186-99.

220. M.R. Johnson., et al., Distribution of relaxin between human maternal and fetal circulations and amniotic fluid. Journal of Endocrinology, 1992. 134, 313-317.

221. H.W. Farber, and J. Loscalzo., Mechanisms of Disease: Pulmonary Arterial Hypertension. The New England Journal of Medicine, 2004. 351, 1655-65.

222 M. Humbert, O. Sitbon, and G. Simonneau, Drug Therapy: Treatment of Pulmonary Arterial Hypertension. The New England Journal of Medicine, 2004. 351, 1425-36.

223. N.F. Voelkel, and J. Gomez-Arroyo, The role of vascular endothelial growth factor in pulmonary arterial hypertension. The angiogenesis paradox. Am J Respir Cell Mol Biol, 2014. 51, 474-84.

224. Q. Du., et al., Nitric oxide production upregulates Wnt/beta-catenin signaling by inhibiting Dickkopf-1. Cancer Res, 2013. 73, 6526-37.

225 C.M. Warboys., et al., Bidirectional cross-regulation between the endothelial nitric oxide synthase and beta-catenin signalling pathways. Cardiovasc Res, 2014. 104, 116-26.

226 J. M. Mei., et al., Matrix metalloproteinase(s) mediate(s) NO-induced dissociation of $\beta$ catenin from membrane bound E-cadherin and formation of nuclear $\beta$-catenin/LEF-1 complex. Carcinogenesis, 2002. 23, 2119-2122.

227. B.S. Chow., et al., Relaxin requires the angiotensin II type 2 receptor to abrogate renal interstitial fibrosis. Kidney Int, 2014. 86, 75-85. 